determined by $\sigma$-overlap density between metal and ligand molecular orbitals.

Acknowledgment. We are indebted to S. Gorter, R. C. M. de Groot, and G. Renes for their assistance with X-ray data collection and magnetic susceptibility data collection. We acknowledge the sponsoring by the Leiden Materials Science Center (Werkgroep Fundamenteel Materiaalonderzoek).

Supplementary Material Available: Table SII, listing anisotropic thermal parameters (1 page); Table SI, listing observed and calculated structure factors (10 pages). Ordering information is given on any current masthead page.

Contribution from the Laboratories of Organic Chemistry, Chemical Physics, Chemical Analysis, and Low Temperature Physics, University of Twente, P.O. Box 217, 7500 AE Enschede, The Netherlands

\title{
Metallomacrocycles: Synthesis, X-ray Structure, Electrochemistry, and ESR Spectroscopy of Mononuclear and Heterodinuclear Complexes
}

\author{
Frank C. J. M. van Veggel, ${ }^{\dagger}$ Sybolt Harkema, ${ }^{\ddagger}$ Martinus Bos, ${ }^{\S}$ Willem Verboom, ${ }^{\dagger}$ \\ Catherina J. van Staveren, ${ }^{\dagger}$ Gerrit J. Gerritsma, $\|$ and David N. Reinhoudt ${ }^{*} \dagger$
}

Received August 26, 1988

\begin{abstract}
A number of novel heterodinucleating ligands have been synthesized by $\mathrm{Ba}^{2+}$-templated (1:1) macrocyclization of the dialdehydes $\mathbf{7 , 8}$, and 20 with the diamines 9 and 21 . The ligands have both a cavity suitable for complexation of transition-metal cations and a cavity for complexation of alkali- or alkaline-earth-metal cations. The resulting barium complexes could be converted into the heterodinuclear complexes 1-3, 10-13, 23-25, and 28 upon reaction with nickel, copper, or zinc acetate. The mono-transition-metal complexes 14-17, 26, 27, and 29 could be obtained by removing the barium salt from the polyether cavity. Their ${ }^{1} \mathrm{H}$ NMR, IR, and mass spectra are discussed, and four heterodinuclear complexes have been analyzed by X-ray crystallography. The nickel/barium complex $3 \cdot \mathrm{MeOH}\left(\mathrm{C}_{35} \mathrm{H}_{44} \mathrm{BaCl}_{2} \mathrm{~N}_{2} \mathrm{NiO}_{19}\right)$ crystallizes in the monoclinic system, space group $P 2{ }_{1} / n$, with $a$ $=15.096$ (4) $\AA, b=20.278$ (4) $\AA, c=13.818$ (4) $\AA, \beta=93.61(1)^{\circ}$, and $Z=4$. The nickel $/$ barium complex 10. $\mathrm{H}_{2} \mathrm{O}$ crystallizes in the triclinic system, space group $P \bar{I}$, with $a=9.096$ (3) $\AA, b=11.491$ (7) $\AA, c=17.119$ (5) $\AA, \alpha=92.38(2)^{\circ}, \beta=102.27$ $(1)^{\circ}, \gamma=98.63(2)^{\circ}$, and $Z=2$. The nickel/sodium complex 14-sodium picrate crystallizes in the monoclinic system, space group $P 2_{1} / c$, with $a=14.697$ (5) $\AA, b=14.574$ (7) $\AA, c=14.782$ (3) $\AA, \beta=96.08(1)^{\circ}$, and $Z=4$. The zinc/barium complex 25.3DMF crystallizes in the monoclinic system, space group $C 2 / c$, with $a=24.301$ (5) $\AA, b=26.232$ (4) $\AA, c=22.468$ (7) $\AA, \beta=119.06$ $(2)^{\circ}$, and $Z=8$. In the complexes $3 \cdot \mathrm{MeOH}, 10 \cdot \mathrm{H}_{2} \mathrm{O}$, and $14 \cdot$ sodium picrate the coordination of the nickel cation is square planar. The zinc cation in 25.3DMF has a square-pyramidal coordination with a DMF molecule at the axial position. The distance between the two metal ions in the complexes $3 \cdot \mathrm{MeOH}, 10 \cdot \mathrm{H}_{2} \mathrm{O}, 14$.sodium picrate, and 25.3DMF is 3.63-3.70 $\AA$. The electrochemical properties of the complexed transition-metal cations in the heterodinuclear complexes and mono-transition-metal cation complexes have been investigated by polarography and cyclic voltammetry. The half-wave potential is dependent on the nature of the transition-metal cation and the mode of coordination. Complexation of alkali-metal $\left(\mathrm{Li}^{+}, \mathrm{Na}^{+}\right.$, and $\mathrm{K}^{+}$) or alkaline-earth-metal $\left(\mathrm{Ba}^{2+}\right)$ cations in the polyether cavity resulted in anodic shifts of the half-wave potential up to $213 \mathrm{mV}$; the bivalent $\mathrm{Ba}^{2+}$ induced the largest shifts. The shifts of the half-wave potential are also dependent on the ring size and rigidity of the polyether cavity. Cyclic voltammetry of the nickel/barium complex 10 and the copper/barium complex 12 revealed a chemically reversible but electrochemically irreversible reduction at scan rates of $0.5-6$ and $1-6 \mathrm{~V} / \mathrm{s}$, respectively. A relatively slow adsorption process was observed for the nickel complex 15 , and the reduction was chemically reversible but electrochemically irreversible at scan rates of $50 \mathrm{mV} / \mathrm{s}$ to $2 \mathrm{~V} / \mathrm{s}$. The zinc/barium complex 25 undergoes an irreversible two-electron reduction at $E_{1 / 2}=-1.466 \mathrm{~V}$, whereas the nickel and copper complexes 10-17, 23, 24, 28, and 29 undergo a one-electron reduction. The ESR spectra of a number of copper containing and heterodinuclear complexes are in line with the redox properties.
\end{abstract}

\section{Introduction}

The area of dinucleating ligands able to form homo- and heterodinuclear complexes is of great interest because of a variety of reasons. ${ }^{1}$ First, they may serve as models for metalloproteins like superoxide dismutase, oxidases, and peptidases. ${ }^{2}$ The dinuclear copper-containing complexes attract a great deal of attention because of the two copper centers in the active site of copper proteins like hemocyanin, which transport $\mathrm{O}_{2}$, and the monooxygenases tyrosinase and dopamine $\beta$-hydroxylase, which incorporate oxygen (from $\mathrm{O}_{2}$ ) into organic substrates. ${ }^{3}$ Very recently Karlin and co-workers ${ }^{4}$ have reported that symmetrical dicopper complexes are able to bind molecular oxygen reversibly at low temperature. Second, such dinuclear complexes may bind and activate small molecules. Floriani and co-workers have shown this beautifully for $\mathrm{CO}_{2}$ and $\mathrm{CO}_{2}$-like molecules. ${ }^{5}$ They prepared acyclic $\left[\mathrm{L}^{2-} \mathrm{Co}^{\mathrm{I}}\right]^{-} \mathrm{M}^{+}(\mathrm{M}=\mathrm{Na}, \mathrm{K}, \mathrm{Cs})$ complexes in which the $\mathrm{Co}^{\mathrm{I}}$ center acts as a Lewis base and the $\mathrm{M}^{+}$center as a Lewis acid. Third, dinuclear complexes can be applied as bifunctional catalysts as was shown by McKenzie and Robson ${ }^{6}$ for a dipalladium complex in the hydration of acetonitrile. They observed a pathway

\footnotetext{
${ }^{\dagger}$ Laboratory of Organic Chemistry.

't Laboratory of Chemical Physics.

Laboratory of Chemical Analysis.

"Laboratory of Low Temperature Physics.
}

involving concerted action of the two metal centers. Both Collman et $a l^{7}$ and Leznoff et al. ${ }^{8}$ have reported the electrocatalytic re-

(1) (a) Casellato, U.; Vigato, P. A.; Vidali, M. Coord. Chem. Rev. 1977 23, 31. (b) Casellato, U.; Vigato, P. A.; Fenton, D. E.; Vidali, M. Chem. Soc. Rev. 1979, 199. (c) Chênevert, R.; D'Astous, L. J. Heterocycl. Chem. 1986, 23, 1785. (d) Mangia, A.; Pelizzi. C.; Pelizzi, G. Acto Crystallogr., Sect. B 1974, 30, 2146. (e) Jazwinski, J.; Lehn, J.-M.; Lilienbaum, D.; Ziessel, R.; Guilhem, J.; Pascard, C. J. Chem. Soc., Chem. Commun. 1987, 1691. (f) Iliopoulos, P.; Murray, K. S.; Robson, R.; Wilson, J.; Williams, G. A. J. Chem. Soc., Dalton Trans. 1987, 1585. (g) Gagné, R. R.; Koval, C. A.; Smith, T. J. J. Am. Chem. Soc $1977,99,8367$.

(2) (a) Kanda, W.; Nakamura, M.; Okawa, H.; Kida, S. Bull. Chem. Soc. Jpn. 1982, 55, 471. (b) van Rijn. J.; Reedijk, J.: Dartmann, M.: Krebs, B. J. Chem. Soc., Dalton Trans. 1987, 2579. (c) Cowan, J. A.; Sanders, J. K. M. J. Chem. Soc., Perkin Trans. I 1987, 2395. (d) Acholla, F. V.; Takusagawa, F.; Mertes, K. B. J. Am. Chem. Soc. 1985, 107, 6902. (e) Suzuki, M.: Uehara, A.: Oshio, H.: Endo, K.: Yanaga, M.; Kida, S.: Saito, K. Bull. Chem. Soc. Jpn. 1987, 60, 3547. (f) Mazurek, W.; Bond, A. M.; Murray, K. S.; O'Connor, M. J.; Wedd, A. G. Inorg. Chem. 1985, 24, 2484. (g) Elliott, C. M.; Jain, N. C.; Cranmer, B. K.; Hamburg, A. W. Inorg. Chem. 1987, 26, 3655. (h) Maruyama, K, Kobayashi, F.; Osuka, A. Chem. Lett. 1987, 821. (i) Noblat, S.; Dietrich-Buchecker, C. O.; Sauvage, J.-P. Tetrahedron Lett. 1987, 28 , 5829. (j) Benzekri, A.; Dubourdeaux, P.; Latour, J.-M.; Laugier, J.; Rey, P. J. Chem. Soc., Chem. Commun. 1987, 1564 . (k) Bailey, N. A.; Fenton, D. E.; Moody, R.: Rodriguez de Barbarin, C. O.; Sciambarella, J. N.; Latour, J. M.; Limosin, D.; McKee, V. J. Chem. Soc., Dalton Trans. 1987, 2519. 


\section{Chart I}

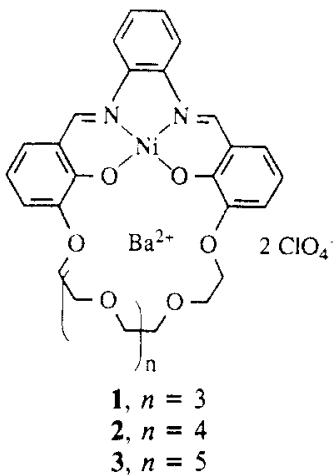

duction of oxygen with cofacial diporphyrins and cofacial diphthalocyanines, respectively.

In principle the behavior of dimetallic complexes can be quite different from that of the monometallic analogues when there is only a small distance between the two metal centers. ${ }^{9}$ Ciampolini et al. ${ }^{10}$ have demonstrated the interaction of two cations in dinickel and dicopper complexes of $\mathrm{N}, \mathrm{N}$-linked bis(cyclam) ligands. The interaction was dependent on the nature of the bridge between the two rings. Mandal et al. ${ }^{11}$ observed two one-electron reductions, two one-electron oxidations, and mixed oxidation states in a symmetrical dicopper(II) complex, which is unusual in such a system.

Hitherto, in the majority of heterodinuclear complexes there have been only combinations of two transition-metal ions and virtually nothing is known about cyclic ligands that have two different cavities, viz. one "hard" cavity that can bind alkali- or alkaline-earth-metal cations and a "soft" cavity capable of binding cations like $\mathrm{Ni}^{2+}, \mathrm{Cu}^{2+}, \mathrm{Co}^{2+}$, and $\mathrm{Zn}^{2+}$. Only a few heterodinucleating cyclic ligands have been reported that have identical cavities. ${ }^{12}$ To the best of our knowledge only Carroy and Lehn ${ }^{12 f}$ have very recently reported dinuclear complexes of cyclic ligands

(3) (a) Pate, J. E.; Cruse, R. W.; Karlin, K. D.; Solomon, E. I. J. Am. Chem. Soc. 1987, 109, 2624 and references cited therein. (b) Karlin, K. D.; Farooq, A.; Hayes, J. C.; Cohen, B. J.; Rowe, T. M.; Sinn, E.; Zubieta, J. Inorg. Chem. 1987, 26, 1271 and references cited therein

(4) (a) Karlin, K. D.; Cruse, R. W.; Gultneh, Y.; Farooq, A.; Hayes, J. C.; Zubieta, J. J. Am. Chem. Soc. 1987, 109, 2668. (b) Ghosh, D.; Tyeklar, Z.; Karlin, K. D.; Jacobson, R. R.; Zubieta, J. J. Am. Chem. Soc. 1987 109, 6889. (c) Karlin, K. D.; Cohen, B. J.; Jacobson, R. R.; Zubieta, J. J. Am. Chem. Soc. 1987, 109, 6194

(5) (a) Gambarotta, S.; Arena, F.; Floriani, C.; Zanazzi, P. F. J. Am. Chem. Soc. 1982, 104, 5082. (b) Arena, F.; Floriani, C.; Chiesi-Villa, A. Guastini, C. Inorg. Chem. 1986, 25, 4589.

(6) McKenzie, C. J.; Robson, R. J. Chem. Soc., Chem. Commun. 1988 112

(7) Collman, J. P.; Hendricks, N. H.; Kim, K.; Bencosme, C. S. J. Chem. Soc., Chem. Commun. 1987, 1537.

(8) Leznoff, C. C.; Lam, H.; Nevin, W. A.; Kobayashi, N.; Janda, P.; Lever A. B. P. Angew. Chem. $1987,99,1065$

(9) (a) Sato, M.: Akabori, S.; Katada, M.; Motoyama, J.; Sano, H. Chem. Lett. 1987, 1847. (b) Himmelsbach, M.; Lintvedt, R. L.; Zehetmair J. K.; Nanny, M.; Heeg, M. J. J. Am. Chem. Soc. 1987, 109, 8003. (c) Adhikary, B.; Mandal, S. K.; Nag, K. J. Chem. Soc., Dalton Trans. 1988, 935.

(10) Ciampolini, M.; Fabbrizzi, L.; Perotti, A.; Poggi, A.; Seghi, B.; Zanobini, F. Inorg. Chem. 1987, 26, 3527.

(11) Mandal, S. K.; Thompson, L. K.; Nag, K.; Charland, J.-P.; Gabe, E. J. Inorg. Chem. 1987, 26, 139

(12) (a) Drew, M. G. B.; McCann, M.; Nelson, S. M. J. Chem. Soc., Dalton Trans. 1981, 1868. (b) Gagnê, R. R.; Henling, L. M.; Kistenmacher, J. T. Inorg. Chem. 1980, 19, 1226. (c) Banci, L.; Bencini, A.; Gatteschi, D. Inorg. Chim. Acta 1979, 36, L419. (d) Gagnê, R. R.; Koval, C. A. Smith, T. J.; Cimolino, M. C. J. Am. Chem. Soc. 1979, 101, 4571. (e) Lambert, S. L.; Spiro, C. L.; Gagnê, R. R.; Hendrickson, D. N. Inorg. Chem. 1982, 21, 68. (f) Carroy, A.; Lehn, J.-M. J. Chem. Soc., Chem. Commun. 1986, 1232. (g) Gagnë, R. R.; Koval, C. A.; Smith, T. J. J. Am. Chem. Soc. 1977, 99, 8367. (h) Hoskins, B. F.: Williams, G. A Aust. J. Chem. 1975, 28, 2593. (i) Hoskins, B. F.; Robson, R.; Williams, G. A. Inorg. Chim. Acta 1976, 16, 121. (j) Diril, H.; Chang, H.-R.; Zhang, X.: Larsen, S. K.; Potenza, J. A.; Pierpont, C. G. Schugar, H. J.: Isied, S. S.; Hendrickson, D. N. J. Am. Chem. Soc 1987, 109.6207. (k) Nelson, J ; Murphy, B. P.; Drew, M. G. B.; Yates, P. C.; Nelson, S. M. J. Chem. Soc., Dalton Trans, 1988, 1001.
Scheme I

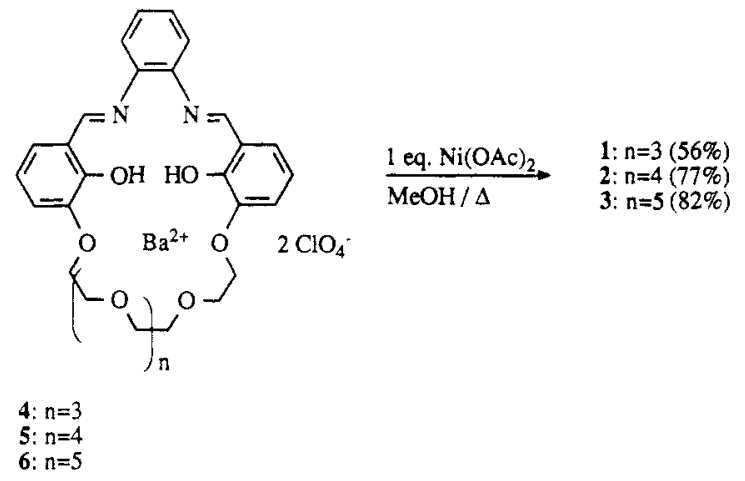

that contain both a transition-metal ion and a non-transition-metal ion $\left(\mathrm{Li}^{+}, \mathrm{Ba}^{2+}, \mathrm{Al}^{3+}\right)$.

A major interest of our group is the complexation of neutral molecules such as urea, nitromethane, and malononitrile with macrocyclic receptor molecules. ${ }^{13}$ In a previous paper we have shown a successful approach for urea complexation by metallomacrocycles in which cations are immobilized as electrophilic centers. ${ }^{14}$ Preliminary results showed that in these metallomacrocycles hard cations can be cocomplexed and this offers an opportunity to study systematically the chemistry of heterodinuclear complexes of cyclic ligands, such as the dinuclear complexes 1-3 (Chart I). ${ }^{15}$ These compounds have a unique combination of two very different cavities, viz. a hard polyether and a soft "salen cype". These dinucleating ligands are able to bring the two different cations in close proximity.

Therefore, we decided to investigate this novel type of heterodinucleating cyclic ligands in detail since our ultimate goal is to use such dimetallic complexes as catalysts. In this paper we report the synthesis of a number of heterodinuclear complexes together with the corresponding mononuclear complexes. Their properties were studied by X-ray crystallography, polarography, cyclic voltammetry, and ESR spectroscopy in order to reveal the possible influence of the hard cations on the physical properties of the complexed transition-metal ions.

\section{Results and Discussion ${ }^{15}$}

Synthesis. Previously we have described the synthesis of the barium complexes 4-6 via a $\mathrm{Ba}^{2+}$-templated macrocyclization of the corresponding dialdehydes and 1,2-benzenediamine in refluxing methanol. ${ }^{14}$ The dinuclear complexes $1-3$ were prepared in $56-82 \%$ yield by reaction of the barium complexes 4-6 with 1 equiv of nickel acetate in refluxing methanol (Scheme I).

The mass spectra of the complexes 1-3 all gave $\mathrm{M}^{+}-\mathrm{Ba}\left(\mathrm{ClO}_{4}\right)_{2}$ as the parent peak, indicating that the nickel is tightly bound. In the ${ }^{1} \mathrm{H}$ NMR spectra (DMSO- $d_{6}$ ) we observed a downfield shift for the imine protons $(\delta 9.55-9.83)$ with respect to the barium complexes 4-6 $(\delta 8.91-8.90)$. In the IR spectra the imine bond

(13) (a) Grootenhuis, P. D. J.; Uiterwijk, J. W. H. M.; Reinhoudt, D. N.; van Staveren, C. J.; Sudhölter, E. J. R.; Bos, M; van Eerden, J. Klooster, W. T.; Kruise, L.; Harkema, S. J. Am. Chem. Soc. 1986, 108 , 780. (b) de Boer, J. A. A.; Uiterwijk, J. W. H. M.; Geevers, J.; Harkema, S.; Reinhoudt, D. N. J. Org. Chem. 1983, 48, 4821. (c) Uiterwijk, J. W. H. M.; van Staveren, C. J.; Reinhoudt, D. N.; den Hertog, H. J., Jr.; Kruise, L.; Harkema, S. J. Org. Chem. 1986, $5 I$, 1575. (d) Aarts, V. M. L. J.; van Staveren, C. J.; Grootenhuis, P. D. J.; van Eerden, J.; Kruise, L.; Harkema, S.; Reinhoudt, D. N. J. Am. Chem. Soc, 1986, 108, 5035. (e) van Staveren, C. J.; Aarts, V. M. L. J.; Grootenhuis, P. D. J.; van Eerden, J.; Harkema, S.; Reinhoudt, D. N. J. Am. Chem. Soc. 1986, 108, 5271. (f) de Boer, J. A. A.; Reinhoudt, D. N.; Harkema, S.; van Hummel, G.; de Jong, F. J. Am. Chem. Soc. 1982, 104, 4073. (g) van Eerden, J.; Grootenhuis, P. D. J.; Dijkstra, P. J.: van Staveren, C. J.; Harkema, S.; Reinhoudt, D. N. $J$. Org. Chem. 1986, 51, 3918.

(14) van Staveren, C. J.; van Eerden, J.; van Veggel, F. C. J. M.; Harkema, S.: Reinhoudt, D. N. J. Am. Chem. Soc. 1988, 110, 4994.

(15) The synthesis of the nickel/barium complex 1 appeared as a preliminary communication: van Staveren, C. J.; Reinhoudt, D. N.; van Eerden, J. Harkema, S. J. Chem. Soc., Chem. Commun. 1987, 974. 
Scheme II

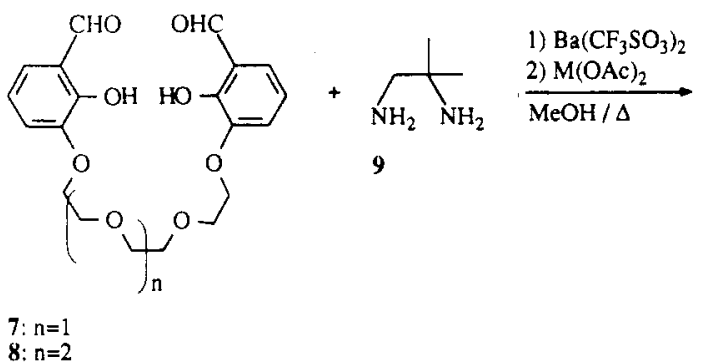

$8: n=2$

exhibited an absorption between 1612 and $1614 \mathrm{~cm}^{-1}$, while for the barium complexes 4-6 these absorptions are located between 1650 and $1625 \mathrm{~cm}^{-1}$, indicating that in the dinuclear complexes the imine linkage has slightly less double-bond character. The solid-state structure of compound 3 was determined by X-ray analysis (vide infra).

For the syntheses of the dinuclear complexes 10-13 a slightly different route was followed, because we expected less stable imine bonds in the mononuclear barium complexes due to the aliphatic 2-methyl-1,2-propanediamine (9) ${ }^{16}$ (Scheme II). The cyclization was carried out by slow addition of both a solution of dialdehyde 7 or $8^{14}$ and a solution of diamine 9 to a refluxing solution of $\mathrm{Ba}\left(\mathrm{CF}_{3} \mathrm{SO}_{3}\right)_{2}$ in methanol. We found that the barium cation is absolutely necessary as a template ${ }^{14}$ in the absence of barium only minor amounts of cyclic product were formed. The resulting barium complex was not isolated, and 1 equiv of nickel acetate or copper acetate was added to the yellow solution, which afforded the solid dinuclear complexes $10-13$ in yields of $71-82 \%$.

The mass spectra of compounds 10-13 showed a parent peak at $\mathrm{M}^{+}-\mathrm{Ba}\left(\mathrm{CF}_{3} \mathrm{SO}_{3}\right)_{2}$, indicating that also in these complexes the transition-metal ion is tightly bound. The absorptions of the imine protons in the ${ }^{1} \mathrm{H}$ NMR spectra (DMSO- $d_{6}$ ) of compounds 10 and 11 show two singlets $(\delta 7.97,7.91$ and $\delta 8.04,7.97$, respectively), reflecting the nonequivalence of these protons. In the IR spectra the imine bond exhibited absorptions between 1624 and $1633 \mathrm{~cm}^{-1}$. The solid-state structure of 10 was determined by X-ray diffraction (vide infra).

The barium-free mononuclear complexes $14-17^{14,17}$ could simply be obtained by stirring a suspension of the dinuclear complex in chloroform with a solution of excess of guanidinium sulfate in water for a few hours. The barium is removed as a barium sulfate precipitate in the aqueous layer, and the complexes 14-17 could be isolated from the organic layer. The solid-state structure of 14. $\mathrm{Na}^{+} \mathrm{Pic}^{-}\left(\mathrm{Na}^{+} \mathrm{Pic}^{-}=\right.$sodium picrate $)$was determined by $\mathrm{X}$-ray crystallography (vide infra).

The dinucleating cyclic ligands described so far have a rather flexible polyethylene glycol unit that forms the hard cavity. In order to make this cavity more rigid, we have incorporated a terphenyl unit (19) developed by $\mathrm{Cram}^{19}$ for the construction of preorganized hemispherands (Scheme III). Upon reaction of the dibromide 19 with 2 equiv of 2,3-dihydroxybenzaldehyde (18) under basic conditions ( $\mathrm{NaH})$ in DMSO, followed by acidic workup and purification by column chromatography, the dialdehyde 20 could be obtained in $23 \%$ yield.

(16) Patai, S., Ed. The Chemistry of the Carbon-Nitrogen Double Bond Interscience: New York, 1970

(17) As a preliminary investigation the association constants of 14 and 16 with alkali-metal cations were determined with the "picrate method" in $\mathrm{CHCl}_{3}$ at $25^{\circ} \mathrm{C}^{18} \log K_{\text {ass }}$ values of 14 for $\mathrm{Li}^{+}, \mathrm{Rb}^{+}$, and $\mathrm{Cs}^{+}$are 7.0, 7.6, and 6.7, respectively. $\log K_{\text {assn }}$ of 16 for $\mathrm{Li}^{+}, \mathrm{Rb}^{+}$, and $\mathrm{Cs}^{+}$are $7.3,8.0$, and 6.9 , respectively. In the case of $\mathrm{Na}^{+}$and $\mathrm{K}^{+}$the dinuclear complexes precipitated from the organic layer.

(18) (a) Lein, G. M.: Cram, D. J. J. Am. Chem. Soc. 1985, 107, 448. (b) Artz, S. P.; deGrandpre, M. P.; Cram, D. J. J. Org. Chem. 1985, 50, 1486 .

(19) Koenig, K. E.; Lein, G. M.; Stuckler, P.; Kaneda, T.; Cram, D. J. J. Am. Chem. Soc. 1979, 101, 3553.
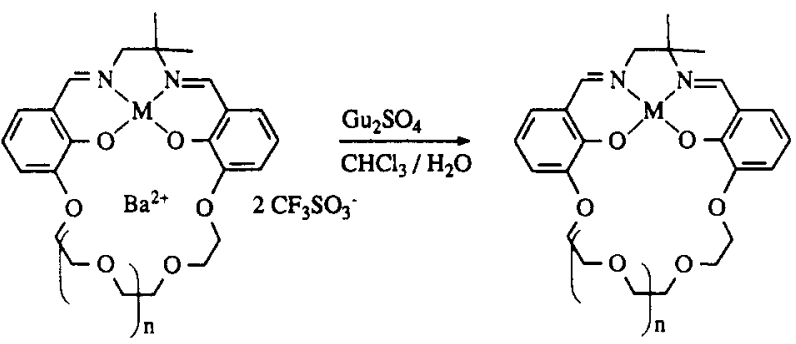

10: $\mathrm{M}=\mathrm{Ni}, \mathrm{n}=1(72 \%)$

11: $\mathrm{M}=\mathrm{Ni}, \mathrm{n}=2(75 \%)$

12: $\mathrm{M}=\mathrm{Cu}, \mathrm{n}=1(82 \%)$

13: $\mathrm{M}=\mathrm{Cu}, \mathrm{n}=2(71 \%)$
14: $\mathrm{M}=\mathrm{Ni}, \mathrm{n}=1$

15: $\mathrm{M}=\mathrm{Ni}, \mathrm{n}=2$

16: $\mathrm{M}=\mathrm{Cu}, \mathrm{n}=1$

17: $M=C u, n=2$
Scheme III<smiles>O=Cc1cccc(O)c1O</smiles>

18<smiles>COc1c(C)cc(C)cc1-c1ccc(C)cc1-c1cc(C)cc(CBr)c1OC</smiles>

$\underset{\mathrm{NMSO} / \mathrm{\pi}}{\longrightarrow}$

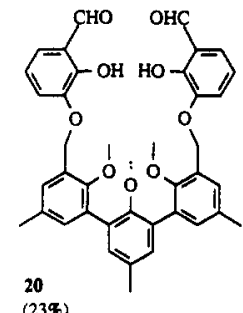

(23\%)
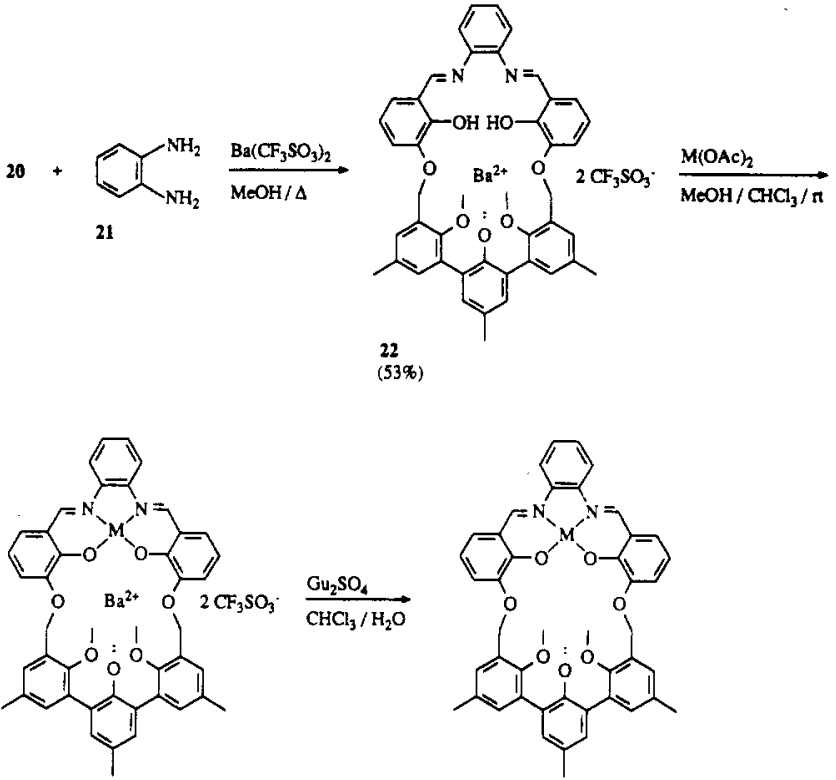

23: $\mathrm{M}=\mathrm{Ni}(67 \%)$

25: $M=\operatorname{Mn}(76 \%)$

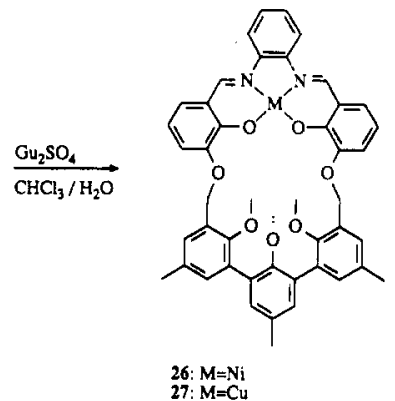

The synthesis of the barium complex 22 was carried out by slow addition of both a solution of 20 and a solution of 1,2-benzenediamine (21) to a refluxing solution of $\mathrm{Ba}\left(\mathrm{CF}_{3} \mathrm{SO}_{3}\right)_{2}$, acting as a template salt, in methanol. After the reaction was completed, the mixture was concentrated to dryness to give an orange solid. The ${ }^{1} \mathrm{H}$ NMR spectrum of this crude product showed an absorption at $\delta 8.47$, indicating that the imine linkage is present, and an $A B$ system for the benzylic protons of the macrocycle 22 at $\delta 5.61$ and $4.77(J=11.4 \mathrm{~Hz})$. Furthermore a broad absorption was observed at $\delta 5.1-5.3$ due to the benzylic protons of noncyclic reaction products. The ratio of cyclic to noncyclic product was approximately $2: 1$. From this crude mixture the barium complex 22 could be isolated in a yield of $53 \% .^{20}$

Reaction of the barium complex 22 with 1 equiv of nickel, copper, and zinc acetates afforded the dinuclear complexes 23-25 in $67-81 \%$ yield. The IR spectra of the dinuclear complexes 23-25

(20) The mass spectrum showed only peaks due to fragmentation. 
Scheme IV
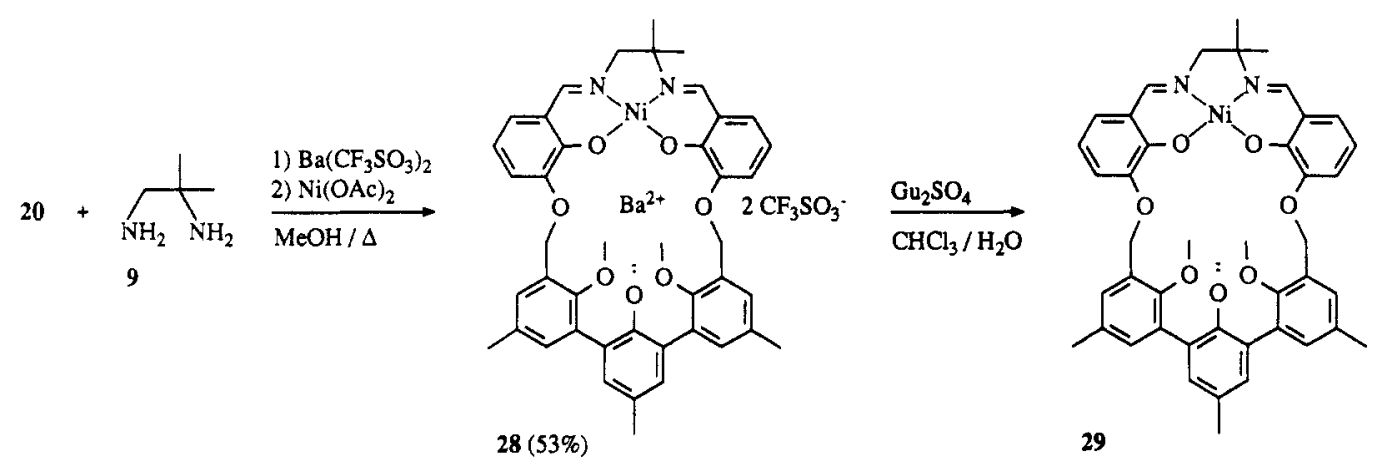

Table I. Metal Coordination in the Crystal Structures: Range of Cation-Coordinating Atom Distances $(\AA)$ and Coordination Number

\begin{tabular}{|c|c|c|c|c|c|c|c|c|}
\hline & \multicolumn{2}{|c|}{ 3.MeOH } & \multicolumn{2}{|c|}{$10 \cdot \mathrm{H}_{2} \mathrm{O}$} & \multicolumn{2}{|c|}{$14 \cdot \mathrm{Na}^{+} \mathrm{Pic}^{-a}$} & \multicolumn{2}{|c|}{ 25.3DMF } \\
\hline & $\mathrm{Ni}^{2+}$ & $\mathrm{Ba}^{2+}$ & $\mathrm{Ni}^{2+}$ & $\mathrm{Ba}^{2+}$ & $\mathrm{Ni}^{2+}$ & $\mathrm{Na}^{+}$ & $\mathrm{Zn}^{2+}$ & $\mathrm{Ba}^{2+}$ \\
\hline coord atom & & & & & & & & \\
\hline $\mathrm{O}_{\text {ether }}$ & & $2.88-3.00$ & & $2.75-2.87$ & & $2.57-2.68$ & & $2.88-3.26$ \\
\hline$O_{\text {phenolate }}$ & $1.87-1.92$ & $2.70-2.73$ & $1.84-1.86$ & $2.71-2.72$ & $1.80-1.81$ & $2.59-2.62$ & $1.96-1.97$ & $2.74-2.78$ \\
\hline $\mathrm{N}$ & $1.87-1.89$ & & $1.83-1.87$ & & $1.78-1.86$ & & $2.07-2.08$ & \\
\hline coord no. & 4 & $11^{b}$ & 4 & $9^{c}$ & 4 & $8^{d}$ & $5^{e}$ & $10^{f}$ \\
\hline
\end{tabular}

${ }^{a} \mathrm{Na}^{+} \mathrm{Pic}^{-}=$sodium picrate. ${ }^{b}+1 \mathrm{ClO}_{4}^{-}\left(\mathrm{Ba}^{2+} \ldots \mathrm{O}=2.80 \AA\right) .{ }^{c}+1 \mathrm{H}_{2} \mathrm{O}\left(\mathrm{Ba}^{2+} \ldots \mathrm{O}=2.78 \AA\right)$ and $2 \mathrm{CF}_{3} \mathrm{SO}_{3}^{-}\left(\mathrm{Ba}^{2+} \ldots \mathrm{O}=2.77-2.82 \AA\right)$. ${ }^{d}+2$ $\mathrm{Pic}^{-}\left(\mathrm{Na}^{+} \ldots \mathrm{O}^{-}=2.40 \AA ; \mathrm{Na}^{+} \ldots \mathrm{O}\left(\mathrm{NO}_{2}\right)=2.60 \AA\right){ }^{e}+1 \mathrm{DMF}\left(\mathrm{Zn}^{2+} \ldots \mathrm{O}=\mathrm{C}=2.04 \AA\right),{ }^{f}+2 \mathrm{DMF}\left(\mathrm{Ba}^{2+} \ldots \mathrm{O}=\mathrm{C}=2.70-2.72 \AA\right)$ and $1 \mathrm{CF}_{3} \mathrm{SO}_{3}^{-}$ $\left(\mathrm{Ba}^{2+} \ldots \mathrm{O}=2.59 \AA\right)$.

showed an absorption for the imine bond at $1614-1615 \mathrm{~cm}^{-1}$, compared with $1623 \mathrm{~cm}^{-1}$ for the barium complex 22, indicating that a metal cation is complexed in the soft cavity. The benzylic protons of 23 and 25 exhibited in the ${ }^{1} \mathrm{H}$ NMR spectra (DMSO- $d_{6}$ ) an AB system at $\delta 5.54$ and $4.98(J=11.4 \mathrm{~Hz})$ and $\delta 5.43$ and $5.09(J=12.2 \mathrm{~Hz})$, respectively. Fast atom bombardment (FAB) mass spectrometry showed in all cases a parent peak corresponding to the molecular weight with loss of one $\mathrm{CF}_{3} \mathrm{SO}_{3}{ }^{-}$anion. The structure of $\mathbf{2 5}$ has also been proven by X-ray analysis (vide infra).

The barium could be removed by stirring a suspension of the dinuclear complex in chloroform with a solution of excess of guanidinium sulfate in water. In the cases of the compounds 23 and 24 the barium-free complexes 26 and 27 , respectively, were obtained in quantitative yields but in the case of $\mathbf{2 5}$ the barium-free complex proved to be unstable, indicating that the zinc cation is less tightly bound than the other two cations. ${ }^{21}$ FAB mass spectra of $\mathbf{2 6}$ and $\mathbf{2 7}$ exhibited peaks at $m / e 791$ and 796, respectively, corresponding to the molecular weight plus one hydrogen. Furthermore peaks at $m / e 813,829$ and $m / e 818,834$, respectively, were observed corresponding to the molecular weight plus uptake of sodium and potassium ions, respectively. ${ }^{22}$ In the ${ }^{1} \mathrm{H}$ NMR spectrum of the nickel complex $\mathbf{2 6}$ an $\mathrm{AB}$ system is present for the benzylic proton at $\delta 5.41$ and $4.20(J=11.4 \mathrm{~Hz})$.

When the IR spectra of the barium-containing (23-25) and the barium-free complexes ( 26 and 27 ) are compared, distinct differences, indicating the presence or absence of barium, could be observed. An example is given in Figure 1. The main features of the differences are indicated with asterisks.

The aliphatic 2-methyl-1,2-propanediamine (9) was also used in the macrocyclization with the dialdehyde $\mathbf{2 0}$, in order to investigate the effect of the incorporation of a rigid polyether cavity, but with the same "salen" cavity for the transition-metal ion. We followed the same procedure as for the synthesis of the compounds 10-13, and the resulting dinuclear complex 28 was isolated in a yield of $53 \%$ (Scheme IV).

The ${ }^{\mathrm{t}} \mathrm{H}$ NMR spectrum $\left(\mathrm{CD}_{3} \mathrm{CN}\right)$ of 28 showed a singlet for the imine protons at $\delta 7.62$ and an $\mathrm{AB}$ system for the benzylic protons at $\delta 5.54$ and $4.96,4.92(J=12.1 \mathrm{~Hz})$. We expected two

(21) The ${ }^{1} \mathrm{H}$ NMR and the IR spectra obtained after removal of the barium from 25 were not in agreement with those of the expected zinc complex. The ligand is probably hydrolyzed.

(22) $\mathrm{Na}^{+} / \mathrm{K}^{+}$complexation has probably taken place in the stainless steel probe with the sample loaded in thioglycerol.
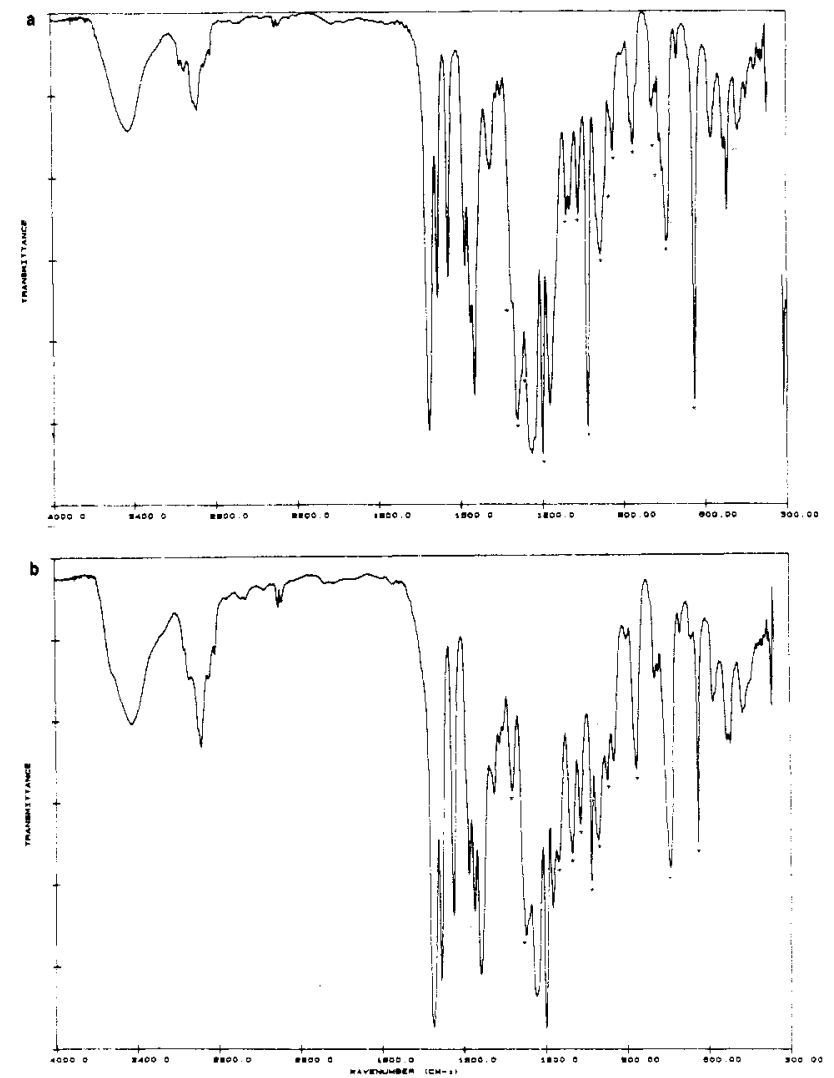

Figure 1. IR spectra $(\mathrm{KBr})$ of $24(\mathrm{a})$ and $27(\mathrm{~b})$.

AB systems for the benzylic protons, but apparently one of the two AB systems has the same chemical shift. We observed two singlets for the methyl groups of the bridge between the two imine bonds ( $\delta 1.47$ and 1.43), which indicates that the terphenyl unit is not rapidly inverting.

The barium-free complex 29 could be obtained via the standard procedure. This compound 29 showed $\mathrm{M}^{+}$as the parent peak in the mass spectrum and a signal at $1622 \mathrm{~cm}^{-1}$ for the imine bond in the IR spectrum. In the ${ }^{1} \mathrm{H}$ NMR spectrum the imine protons showed a singlet at $\delta 7.37$ and the benzylic protons an $\mathrm{AB}$ system at $\delta 5.54$ and $4.65,4.62(J=11.4 \mathrm{~Hz})$. The lack of symmetry in 29 is further reflected in the two different singlets for the methyl 

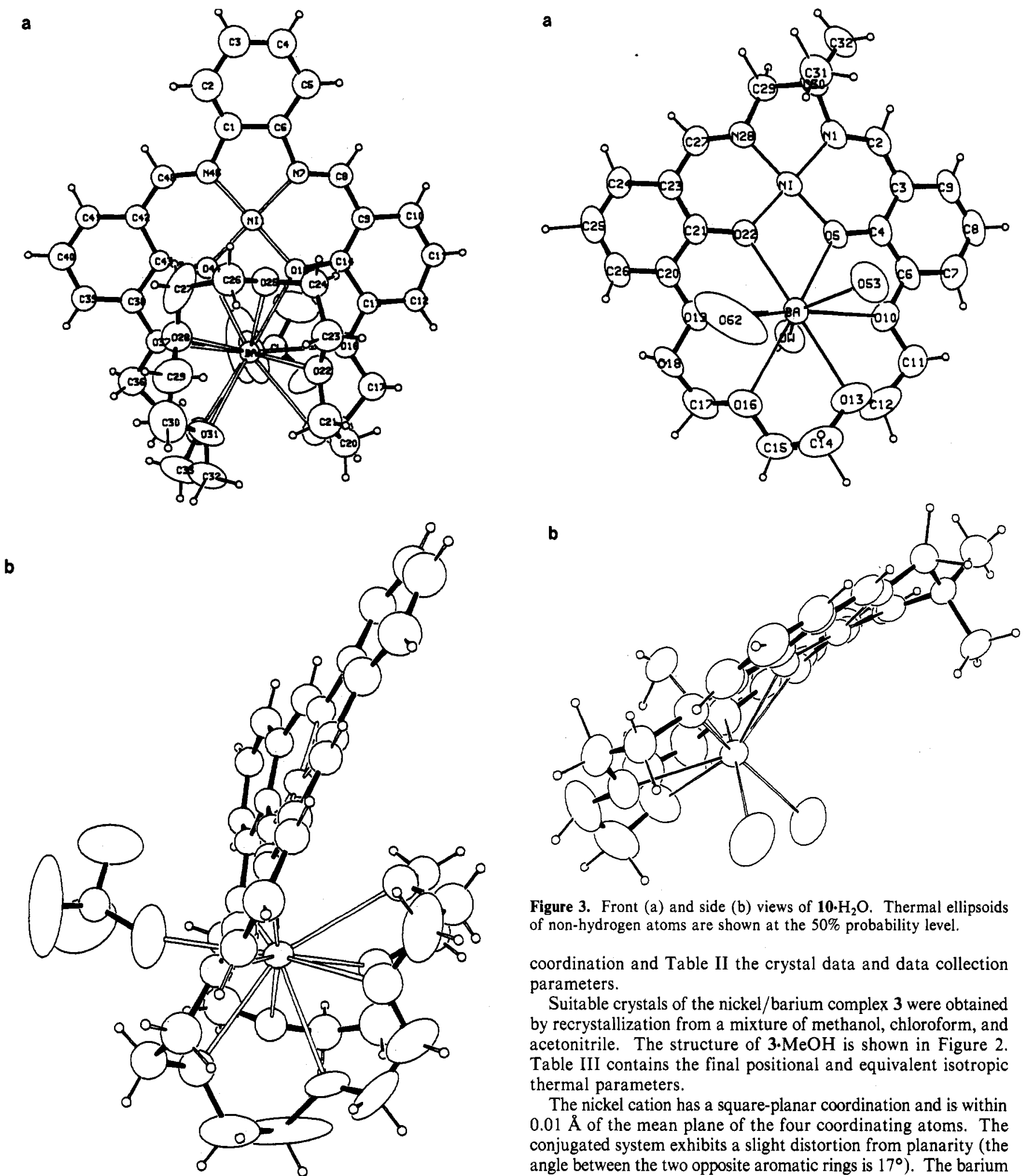

Figure 3. Front (a) and side (b) views of $\mathbf{1 0} \cdot \mathrm{H}_{2} \mathrm{O}$. Thermal ellipsoids of non-hydrogen atoms are shown at the $50 \%$ probability level.

coordination and Table II the crystal data and data collection parameters.

Suitable crystals of the nickel/barium complex 3 were obtained by recrystallization from a mixture of methanol, chloroform, and acetonitrile. The structure of $3 \cdot \mathrm{MeOH}$ is shown in Figure 2. Table III contains the final positional and equivalent isotropic thermal parameters.

The nickel cation has a square-planar coordination and is within $0.01 \AA$ of the mean plane of the four coordinating atoms. The conjugated system exhibits a slight distortion from planarity (the angle between the two opposite aromatic rings is $17^{\circ}$ ). The barium cation is complexed in the polyether cavity. The polyether chain is completely folded around the barium in order to allow the coordination of the two phenolate oxygens $\left(\mathrm{Ba}^{2+} \ldots \mathrm{O}=2.70-2.73\right.$ $\AA$ ) and eight ether oxygens $\left(\mathrm{Ba}^{2+} \ldots \mathrm{O}=2.88-3.00 \AA\right)$. The 11 th coordination site is occupied by a perchlorate anion $\left(\mathrm{Ba}^{2+} \ldots \mathrm{O}=\right.$ $2.80 \AA$ ). A similar folding of the macrocycle around the barium has been observed in the structure of compound $1^{14}$ and in the complex of dibenzo-24-crown-8 with barium perchlorate. ${ }^{24}$ The nickel-barium distance is $3.70 \AA$.

Single crystals of the nickel/barium complex 10 were obtained by slow saturation of a solution of $\mathbf{1 0}$ in acetonitrile with diiso-

(23) Johnson, C. K. "ORTEP", Report ORNL-3794; Oak Ridge National Laboratory: Oak Ridge, TN, 1965.

(24) Hughes, D. L.; Mortimer, C. L.; Truter, M. R. Acta Crystallogr., Sect. $B$ 1978, 34, 800 . 
Table II. Crystal Data and Data Collection Parameters

\begin{tabular}{|c|c|c|c|c|}
\hline & 3. $\mathrm{MeOH}$ & $10 \cdot \mathrm{H}_{2} \mathrm{O}$ & 14. $\mathrm{Na}^{+} \mathrm{Pic}^{-}$ & 25.3DMF \\
\hline $\begin{array}{l}\text { formula } \\
\text { fw }\end{array}$ & $\begin{array}{l}\mathrm{C}_{35} \mathrm{H}_{44} \mathrm{BaCl}_{2} \mathrm{~N}_{2} \mathrm{NiO}_{19} \\
1063.70\end{array}$ & $\begin{array}{l}\mathrm{C}_{26} \mathrm{H}_{30} \mathrm{BaF}_{6} \mathrm{~N}_{2} \mathrm{NiO}_{13} \mathrm{~S}_{2} \\
952.69\end{array}$ & $\begin{array}{l}\mathrm{C}_{30} \mathrm{H}_{30} \mathrm{~N}_{5} \mathrm{NaNiO}_{13} \\
750.29\end{array}$ & $\begin{array}{l}\mathrm{C}_{57} \mathrm{H}_{61} \mathrm{BaF}_{6} \mathrm{~N}_{5} \mathrm{O}_{16} \mathrm{~S}_{2} \mathrm{Zn} \\
1452.97\end{array}$ \\
\hline lattice type & monoclinic & triclinic & monoclinic & monoclinic \\
\hline space group & $P 2_{1} / n$ & $P \bar{l}$ & $P 2_{1} / c$ & $C 2 / c$ \\
\hline$T, \mathrm{~K}$ & 293 & 293 & 293 & 173 \\
\hline$a, \AA$ & $15.096(4)$ & $9.096(3)$ & $14.697(5)$ & $24.301(5)$ \\
\hline$b, \AA$ & $20.278(4)$ & $11.491(7)$ & $14.574(7)$ & $26.232(4)$ \\
\hline$c, \AA$ & $13.818(4)$ & $17.119(5)$ & $14.782(3)$ & $22.468(7)$ \\
\hline$\alpha, \operatorname{deg}$ & & $92.38(2)$ & & \\
\hline$\beta$, deg & $93.61(1)$ & $102.27(1)$ & $96.08(1)$ & $119.06(2)$ \\
\hline$\gamma, \mathrm{deg}$ & & $98.63(2)$ & & \\
\hline$V, \AA^{3}$ & $4222(3)$ & $1724(3)$ & $3148(3)$ & $12519(10)$ \\
\hline $\mathrm{Z}$ & 4 & 2 & 4 & 8 \\
\hline$D_{\mathrm{s}}, \mathrm{g} / \mathrm{cm}^{3}$ & 1.67 & 1.84 & 1.58 & 1.55 \\
\hline$F(000)$ & 2152 & 948 & 1552 & 5904 \\
\hline$\mu, \mathrm{cm}^{-1}$ & 15.7 & 18.9 & 7.1 & 11.5 \\
\hline$\theta$ range, deg & $3-22.5$ & $2-25$ & $3-22$ & $3-22.5$ \\
\hline data collected & $\pm h,+k,+l$ & $\pm h, \pm k,+l$ & $\pm h,+k,+l$ & $\pm h,+k,+l$ \\
\hline no. of unique reflns measd & 5430 & 6053 & 3887 & 10220 \\
\hline no. of reflns observed $[I>3 \sigma(I)]$ & 2645 & 4837 & 1353 & 5528 \\
\hline no. of variables & 342 & 573 & 240 & 518 \\
\hline$R, \%$ & 8.1 & 5.9 & 10.9 & 10.0 \\
\hline$R_{\mathrm{w}}, \%$ & 9.2 & 7.2 & 11.4 & 8.5 \\
\hline weighting factor $p$ & 0.04 & 0.04 & 0.04 & 0.04 \\
\hline extinction $g$ & $0.72 \times 10^{-7}$ & & $0.22 \times 10^{-7}$ & \\
\hline
\end{tabular}

propyl ether. ${ }^{25}$ The structure of $\mathbf{1 0} \cdot \mathrm{H}_{2} \mathrm{O}$ is shown in Figure 3. Table IV contains the final positional and equivalent isotropic thermal parameters.

he nickel cation has a square-planar coordination and is within $0.01 \AA$ of the mean plane of the four coordinating atoms. The angle between the two aromatic rings is $9.5^{\circ}$. The barium cation is 9 -fold coordinated by two phenolic oxygens $\left(\mathrm{Ba}^{2+} \ldots \mathrm{O}=\right.$ $2.71-2.72 \AA)$, four ether oxygens $\left(\mathrm{Ba}^{2+} \ldots \mathrm{O}=2.75-2.87 \AA\right)$, two triflate $\left(\mathrm{CF}_{3} \mathrm{SO}_{3}{ }^{-}\right)$anions $\left(\mathrm{Ba}^{2+} \ldots \mathrm{O}=2.77-2.82 \AA\right)$, and one water molecule $\left(\mathrm{Ba}^{2+} \ldots \mathrm{O}=2.78 \AA\right.$ ). The crown ether cavity is obviously too small for barium, and therefore the barium ion is complexed in a perching fashion. The six crown ether oxygens are within $0.36 \AA$ of their mean plane. The barium is displaced by $0.84 \AA$ out of this plane toward the two triflate anions. At the other face one water molecule occupies the apical position. This combination of equatorial coordination by a crown ether and apical coordination by anions and possibly a water molecule has been observed previously for barium. ${ }^{14,26}$ In all cases there is an approximate hexagonal-planar coordination, with three or four apical ligands. If the cavity is even smaller, the displacement will be larger, and optimal coordination is then achieved by sandwiching the cation with two hosts. ${ }^{27}$ The distance between the two metal ions in $\mathbf{1 0} \cdot \mathrm{H}_{2} \mathrm{O}$ is $3.69 \AA$.

Rather small ruby red crystals of compound $14 \cdot \mathrm{Na}^{+} \mathrm{Pic}^{-28}$ were grown by slow diffusion of a solution of sodium picrate in methanol into a solution of $\mathbf{1 4}$ in chloroform. The structure of this dinuclear complex is shown in Figure 4. Table V contains the final positional and equivalent isotropic thermal parameters.

In this complex the nickel cation also has a square-planar coordination. It is within $0.03 \AA$ of the mean plane of the four coordinating atoms. The methylene group in the bridge between the two imine bonds is probably disordered, as is the carbon atom of one imine linkage. The angle between the two aromatic rings is $15^{\circ}$. The six macrocyclic oxygens are within $0.04 \AA$ of their

(25) Jones, P. G. Chem, Br, 1981, 17,222.

(26) (a) Drew, M. G. B.; Nelson, S. M. J. Chem. Soc., Dalton Trans. 1981, 1678. (b) Dyer, R. B.; Metcalf, D. H.; Ghirardelli, R. G.; Dalmer, R. A.; Holt, E. M. J. Am. Chem. Soc. 1986, 108, 3621. (c) Wei, Y. Y.; Tinant, B.: Declercq, J.-P.; van Meerssche, M. Acta Crystallogr. Sect. $C$ 1988, 44, 77. (d) Dalley, N. K.; Sypherd, D.; George, R. D. $J$. Heterocycl. Chem. 1984, 2l, 497.

(27) (a) Nelson, S. M.; Esho, F. S.; Drew, M. G. B. J. Chem. Soc., Dalton Trans. 1983, 1857. (b) Wei, Y. Y.; Tinant. B.; Declercq, J.-P.; van Meerssche, M. Acta Crystallogr, Sect. C 1988, 44, 68.

(28) Anal. Calcd for 14. $\mathrm{Na}^{+} \mathrm{Pic}^{-}\left(\mathrm{C}_{30} \mathrm{H}_{30} \mathrm{~N}_{5} \mathrm{NaNiO}_{13}\right): \mathrm{C}, 48.03 ; \mathrm{H}, 4.03$; $\mathrm{N}, 9.33$. Found: $\mathrm{C}, 47.83 ; \mathrm{H}, 3.95 ; \mathrm{N}, 9.37$

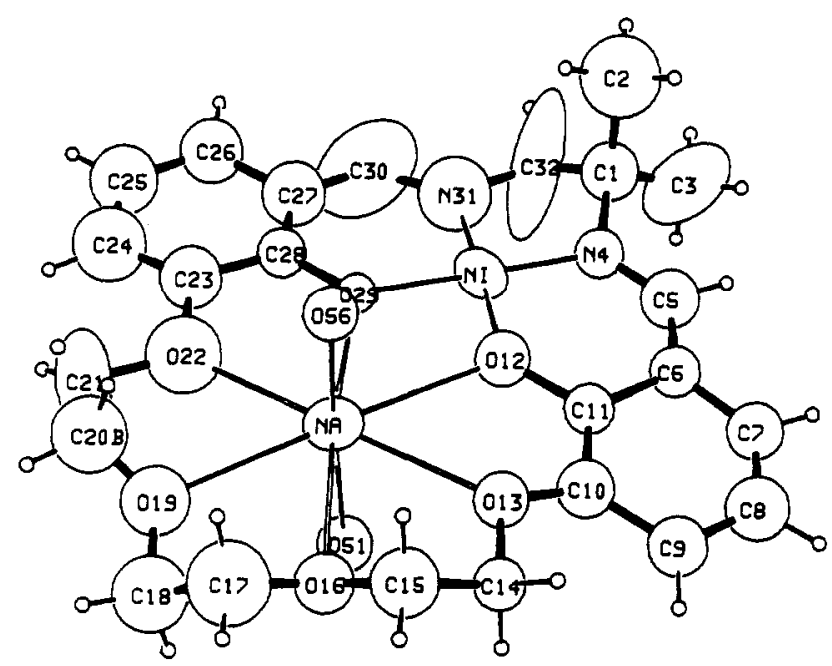

Figure 4. View of $14 \cdot \mathrm{Na}^{+} \mathrm{Pic}^{-}$. Only the coordinated oxygens of the two picrate anions are shown for clarity. Thermal ellipsoids of non-hydrogen atoms are shown at the 50\% probability level. Carbon atom C20 was found at two positions, but for clarity only C20B is shown.

mean plane. The sodium cation is only displaced $0.13 \AA$ out of this plane, giving an encapsulated complex. The two apical positions are occupied by two picrate anions, one coordinating with the phenolic oxygen $\left(\mathrm{Na}^{+} \ldots \mathrm{O}=2.40 \AA\right)$ and the other with an oxygen of a $p$-nitro group $\left(\mathrm{Na}^{+} \ldots \mathrm{O}=2.60 \AA\right)$. Such an encapsulated hexagonal-planar coordination of sodium with two apical ligands is also observed for sodium with 18 -crown-6. ${ }^{29}$ The distance between the two phenolate oxygens $(2.43 \AA)$ is somewhat smaller than for the compounds $3 \cdot \mathrm{MeOH}, 10 \cdot \mathrm{H}_{2} \mathrm{O}$, and $14 \cdot \mathrm{H}_{2} \mathrm{O}^{14}$ $(2.52,2.51$, and $2.51 \AA$, respectively), so the sodium seems to induce a slight contraction of the ligand. The contraction is also reflected in the somewhat shorter distances of the four atoms complexing the nickel cation (Table I). The distance between the two metal ions is $3.63 \AA$.

(29) (a) Darensbourg, D. J.; Bauch, C. G.; Reingold, A. L. Inorg. Chem. 1987, 26, 977. (b) Cooper, M. K.; Duckworth, P. A.; Henrick, K.; McPartlin, M. J. Chem. Soc, Dalton Trans, 1981, 2357. (c) Sheldrick, W. S.; Kroner, J.; Zwaschka, F.; Schmidpeter, A. Angew. Chem. 1979 91, 998. (d) Bailey, S. I.; Engelhardt, L. M.; Leung, W.-P.; Raston, C. L.; Ritchie, I. M.; White, A. H. J. Chem. Soc., Dalton Trans. 1985, 1747 . 
Table III. Final Positional Parameters and Equivalent Isotropic Thermal Parameters for 3. $\mathrm{MeOH}^{a}$

\begin{tabular}{|c|c|c|c|c|c|c|c|c|c|}
\hline atom & $x$ & $y$ & $z$ & $B, \AA^{2}$ & atom & $x$ & $y$ & $z$ & $B, \AA^{2}$ \\
\hline $\mathrm{Bal}$ & $0.20092(7)$ & $0.12573(6)$ & $0.23892(9)$ & $3.01(2)$ & C35 & $0.016(1)$ & $0.102(1)$ & $0.406(2)$ & $4.7(5)^{*}$ \\
\hline $\mathrm{Nil}$ & $0.1843(2)$ & 0.0423 (1) & $-0.0000(2)$ & $3.08(6)$ & $\mathrm{C} 36$ & $-0.040(1)$ & $0.121(1)$ & $0.312(1)$ & $5.2(5)^{*}$ \\
\hline $\mathrm{Cll}$ & $0.8259(3)$ & 0.0636 & $0.6474(4)$ & $4.3(1)$ & C38 & $-0.034(1)$ & $0.108(1)$ & 0.139 (1) & $3.9(5)^{*}$ \\
\hline $\mathrm{Cl} 2$ & $0.3577(4)$ & -0.1914 & $-0.2411(5)$ & $6.4(2)$ & C39 & $-0.120(1)$ & $0.130(1)$ & $0.120(1)$ & $4.1(4)^{*}$ \\
\hline 015 & $0.2621(7)$ & $0.0340(6)$ & $0.1143(9)$ & $3.1(3)^{*}$ & $\mathrm{C} 40$ & -0.158 (1) & 0.129 (1) & $0.029(2)$ & $4.9(5)^{*}$ \\
\hline O16 & $0.3550(8)$ & $0.0391(7)$ & 0.279 (1) & $4.1(3)^{*}$ & C41 & -0.110 (1) & $0.110(1)$ & $-0.045(1)$ & $3.6(4)^{*}$ \\
\hline 019 & $0.3295(8)$ & $0.1479(7)$ & 0.398 (1) & $4.3(3)^{*}$ & $\mathrm{C} 42$ & $-0.022(1)$ & 0.089 (1) & $-0.034(2)$ & $3.7(4)^{*}$ \\
\hline $\mathrm{O} 22$ & $0.3613(9)$ & $0.2017(8)$ & $0.224(1)$ & $4.9(3)^{*}$ & $\mathrm{C} 43$ & $0.018(1)$ & $0.0890(9)$ & $0.063(1)$ & $2.8(4)^{*}$ \\
\hline $\mathrm{O} 25$ & $0.2613(9)$ & $0.1802(8)$ & $0.052(1)$ & $4.8(3)^{*}$ & C45 & $0.028(1)$ & 0.073 (1) & $-0.112(1)$ & $3.6(4) *$ \\
\hline $\mathrm{O} 28$ & $0.1140(9)$ & $0.2389(8)$ & $0.132(1)$ & $5.5(4)^{*}$ & C61 & $0.352(3)$ & $0.181(2)$ & 0.681 & $15(1)$ \\
\hline $\mathrm{O} 31$ & $0.162(1)$ & $0.2494(8)$ & $0.333(1)$ & $6.3(4)$ & $\mathrm{H} 2$ & 0.062 & 0.076 & -0.303 & $5.0^{*}$ \\
\hline $\mathrm{O} 34$ & $0.0957(8)$ & $0.1364(7)$ & $0.411(1)$ & $4.6(3)^{*}$ & $\mathrm{H} 3$ & 0.138 & 0.034 & -0.436 & $5.0^{*}$ \\
\hline $\mathrm{O} 37$ & $0.0099(7)$ & $0.1068(6)$ & $0.2303(9)$ & $3.4(3)^{*}$ & $\mathrm{H} 4$ & 0.274 & -0.010 & -0.408 & $5.0^{*}$ \\
\hline O44 & $0.1041(7)$ & $0.0732(6)$ & $0.0874(9)$ & $3.0(3)^{*}$ & H5 & 0.336 & -0.031 & -0.254 & $5.0^{*}$ \\
\hline O51 & $0.841(1)$ & $-0.0069(9)$ & $0.671(2)$ & $10.2(6)$ & H8 & 0.366 & -0.045 & -0.109 & $5.0^{*}$ \\
\hline 052 & $0.791(2)$ & 0.092 & $0.724(2)$ & $12.5(7)$ & H10 & 0.481 & -0.090 & 0.001 & $5.0^{*}$ \\
\hline O53 & 0.759 & 0.074 (1) & $0.576(2)$ & $13.3(8)$ & H11 & 0.548 & -0.096 & 0.153 & $5.0^{*}$ \\
\hline O54 & 0.904 (1) & 0.091 (1) & $0.635(2)$ & $19(1)$ & $\mathrm{H} 12$ & 0.508 & -0.027 & 0.279 & $5.0^{*}$ \\
\hline O60 & $0.141(1)$ & 0.708 (1) & $0.725(2)$ & $14.6(8)$ & $\mathrm{H} 17 \mathrm{~A}$ & 0.462 & 0.059 & 0.358 & $5.0^{*}$ \\
\hline O61 & 0.268 (1) & $-0.182(1)$ & $-0.284(2)$ & $10.7(7)$ & $\mathrm{H} 17 \mathrm{~B}$ & 0.413 & -0.003 & 0.395 & $5.0^{*}$ \\
\hline O62 & 0.391 (1) & $-0.254(1)$ & $-0.260(2)$ & $10.0(6)$ & $\mathrm{H} 18 \mathrm{~A}$ & 0.391 & 0.098 & 0.493 & $5.0^{*}$ \\
\hline O63 & $0.361(2)$ & $-0.173(2)$ & $-0.144(2)$ & $17(1)$ & H18B & 0.301 & 0.067 & 0.456 & $5.0^{*}$ \\
\hline O64 & $0.411(1)$ & $-0.147(1)$ & $-0.279(2)$ & $19(1)$ & $\mathrm{H} 20 \mathrm{~A}$ & 0.456 & 0.172 & 0.374 & $5.0^{*}$ \\
\hline N7 & $0.2639(9)$ & $0.0058(8)$ & $-0.083(1)$ & $3.2(3)^{*}$ & $\mathrm{H} 20 \mathrm{~B}$ & 0.409 & 0.218 & 0.445 & $5.0^{*}$ \\
\hline N45 & 0.109 (1) & $0.0559(8)$ & $-0.113(1)$ & $3.6(3)^{*}$ & $\mathrm{H} 21 \mathrm{~A}$ & 0.432 & 0.269 & 0.293 & $5.0^{*}$ \\
\hline $\mathrm{Cl}$ & $0.147(1)$ & 0.040 (1) & $-0.201(2)$ & $4.8(5)^{*}$ & $\mathrm{H} 21 \mathrm{~B}$ & 0.333 & 0.266 & 0.318 & $5.0^{*}$ \\
\hline $\mathrm{C}_{2}$ & $0.115(2)$ & $0.050(1)$ & $-0.293(2)$ & $6.2(6)^{*}$ & $\mathrm{H} 23 \mathrm{~A}$ & 0.453 & 0.229 & 0.138 & $5.0^{*}$ \\
\hline C3 & $0.163(1)$ & $0.030(1)$ & $-0.372(2)$ & $6.3(6)^{*}$ & $\mathrm{H} 23 \mathrm{~B}$ & 0.367 & 0.271 & 0.125 & $5.0^{*}$ \\
\hline $\mathrm{C} 4$ & $0.243(1)$ & $0.001(1)$ & $-0.353(2)$ & $5.3(6)^{*}$ & $\mathrm{H} 24 \mathrm{~A}$ & 0.379 & 0.142 & 0.063 & $5.0^{*}$ \\
\hline C5 & 0.279 & $-0.010(1)$ & $-0.263(2)$ & $5.0(5)^{*}$ & $\mathrm{H} 24 \mathrm{~B}$ & 0.371 & 0.202 & -0.007 & $5.0^{*}$ \\
\hline C6 & $0.234(1)$ & 0.008 & $-0.183(1)$ & $3.5(4)^{*}$ & $\mathrm{H} 26 \mathrm{~A}$ & 0.225 & 0.241 & -0.056 & $5.0^{*}$ \\
\hline $\mathrm{C} 8$ & $0.335(1)$ & $-0.025(1)$ & -0.059 & $4.0(5)^{*}$ & $\mathrm{H} 26 \mathrm{~B}$ & 0.246 & 0.278 & 0.041 & $5.0^{*}$ \\
\hline $\mathrm{C} 9$ & $0.375(1)$ & $-0.030(1)$ & 0.038 (1) & $3.4(4)^{*}$ & $\mathrm{H} 27 \mathrm{~A}$ & 0.100 & 0.198 & 0.005 & $5.0^{*}$ \\
\hline $\mathrm{ClO}$ & $0.456(1)$ & -0.069 (1) & $0.054(2)$ & $4.2(5)^{*}$ & $\mathrm{H} 27 \mathrm{~B}$ & 0.097 & 0.275 & 0.002 & $5.0^{*}$ \\
\hline $\mathrm{Cll}$ & $0.498(1)$ & -0.067 (1) & $0.142(1)$ & $3.8(4)^{*}$ & $\mathrm{H} 29 \mathrm{~A}$ & 0.095 & 0.329 & 0.147 & $5.0^{*}$ \\
\hline $\mathrm{C} 12$ & $0.472(1)$ & -0.031 (1) & 0.220 (1) & $3.3(4)^{*}$ & $\mathrm{H} 29 \mathrm{~B}$ & 0.190 & 0.305 & 0.174 & $5.0^{*}$ \\
\hline $\mathrm{C} 13$ & 0.389 (1) & 0.002 & $0.207(1)$ & $2.9(4)^{*}$ & $\mathrm{H} 30 \mathrm{~A}$ & 0.052 & 0.293 & 0.281 & $5.0^{*}$ \\
\hline $\mathrm{Cl} 4$ & 0.339 (1) & 0.002 & 0.114 (1) & $2.9(4)^{*}$ & $\mathrm{H} 30 \mathrm{~B}$ & 0.132 & 0.340 & 0.299 & $5.0^{*}$ \\
\hline $\mathrm{Cl} 7$ & $0.406(1)$ & 0.041 (1) & $0.369(2)$ & $5.2(5)^{*}$ & $\mathrm{H} 32 \mathrm{~A}$ & 0.145 & 0.283 & 0.458 & $5.0^{*}$ \\
\hline $\mathrm{C} 18$ & $0.352(2)$ & 0.089 (1) & $0.437(2)$ & $6.0(6)^{*}$ & $\mathrm{H} 32 \mathrm{~B}$ & 0.206 & 0.221 & 0.466 & $5.0^{*}$ \\
\hline $\mathrm{C}_{2} \mathrm{O}$ & $0.402(1)$ & 0.194 (1) & 0.385 & $5.2(5)^{*}$ & $\mathrm{H} 33 \mathrm{~A}$ & 0.033 & 0.220 & 0.418 & $5.0^{*}$ \\
\hline $\mathrm{C} 21$ & $0.383(2)$ & $0.241(1)$ & $0.305(2)$ & $7.1(7)^{*}$ & $\mathrm{H} 33 \mathrm{~B}$ & 0.079 & 0.201 & 0.517 & $5.0^{*}$ \\
\hline $\mathrm{C} 23$ & $0.390(1)$ & 0.228 & $0.135(2)$ & $5.9(6)^{*}$ & $\mathrm{H} 35 \mathrm{~A}$ & -0.016 & 0.112 & 0.462 & $5.0^{*}$ \\
\hline $\mathrm{C} 24$ & $0.354(1)$ & 0.185 (1) & $0.054(2)$ & $4.7(5)^{*}$ & H35B & 0.028 & 0.056 & 0.404 & $5.0^{*}$ \\
\hline C26 & 0.218 & $0.240(1)$ & $0.012(2)$ & $6.0(6)^{*}$ & H36A & -0.056 & 0.166 & 0.315 & $5.0^{*}$ \\
\hline $\mathrm{C} 27$ & 0.125 (1) & $0.237(1)$ & $0.032(2)$ & $8.6(8)$ & H36B & -0.093 & 0.094 & 0.308 & $5.0^{*}$ \\
\hline C 29 & $0.129(2)$ & $0.296(1)$ & $0.178(2)$ & $8.5(8)$ & H39 & -0.152 & 0.144 & 0.174 & $5.0^{*}$ \\
\hline C30 & $0.113(2)$ & $0.298(1)$ & $0.274(2)$ & $8.4(9)$ & $\mathrm{H} 40$ & -0.217 & 0.145 & 0.016 & $5.0^{*}$ \\
\hline C 32 & $0.154(2)$ & $0.239(2)$ & $0.435(2)$ & $17.5(9)$ & $\mathrm{H} 41$ & -0.138 & 0.109 & -0.109 & $5.0^{*}$ \\
\hline C33 & $0.086(2)$ & $0.203(1)$ & $0.450(2)$ & $10.0(9)$ & H45 & -0.004 & 0.074 & -0.174 & $5.0^{*}$ \\
\hline
\end{tabular}

${ }^{a}$ Starred values indicate atoms refined isotropically. Anisotropically refined atoms are given in the form of the isotropic equivalent displacement parameter defined as ${ }^{4} / 3_{3}\left[a^{2} B_{11}+b^{2} B_{22}+c^{2} B_{33}+a b(\cos \gamma) B_{12}+a c(\cos \beta) B_{13}+b c(\cos \alpha) B_{23}\right]$.

At room temperature unstable single crystals of the zinc/barium complex 25 were grown by slow saturation of a solution of $\mathbf{2 5}$ in a mixture of acetone, acetonitrile, and dimethylformamide with diethyl ether..$^{25}$ A crystal mounted and slowly cooled to $-100^{\circ} \mathrm{C}$ proved stable during the data collection. The structure of 25 . 3DMF is shown in Figure 5. Table VI contains the final positional and equivalent isotropic thermal parameters.

In contrast to the square-planar coordination of nickel and copper observed in $3 \cdot \mathrm{MeOH}, 10 \cdot \mathrm{H}_{2} \mathrm{O}, 14 \cdot \mathrm{H}_{2} \mathrm{O},{ }^{14} 14 \cdot \mathrm{Na}^{+} \mathrm{Pic}^{-}$, and 17. $\mathrm{H}_{2} \mathrm{O}^{30}$ the zinc cation has a square-pyramidal coordination that is formed by the two nitrogen atoms $\left(\mathrm{Zn}^{2+} \ldots \mathrm{N}=2.07-2.08 \AA\right)$, the two phenolate oxygens $\left(\mathrm{Zn}^{2+} \ldots \mathrm{O}=1.96-1.97 \AA\right)$ of the macrocycle, and an oxygen of dimethylformamide ${ }^{31}$ at the axial position $\left(\mathrm{Zn}^{2+} \ldots \mathrm{O}=\mathrm{C}=2.04 \AA\right)$. The zinc cation is displaced by $0.39 \AA$ out of the mean plane of the four coordinating atoms

(30) Unpublished resuits.

(31) The presence of DMF was also observed in the mass spectrum (a peak corresponding to the molecular weight with the correct fragmentation pattern for DMF was present) and in the ${ }^{1} \mathrm{H}$ NMR spectrum [ $\delta 7.97$

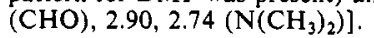

of the macrocycle toward the coordinated carbonyl of DMF. It may thus be concluded that, in contrast to the nickel and copper complexes, the zinc cation complexed in this soft cavity is still sufficiently electrophilic to bind a ligand in the axial position. This type of square-pyramidal coordination of zinc was also recently reported by Brennan and Scheidt ${ }^{32}$ for a zinc porphyrin complex. The displacement of zinc out of the mean plane of the four coordinating nitrogen atoms of the ligand is $0.42 \AA$, and the axial position is occupied by an imidazole molecule. The conjugated system in 25.3DMF shows a larger deviation from planarity than the other complexes reported in this paper; the angle between the two opposite aromatic rings is $39^{\circ}$. This is also reflected in the longer distance between the two phenolate oxygens $(2.86 \AA)$. The barium cation is complexed in the polyether cavity by the two phenolate oxygens $\left(\mathrm{Ba}^{2+} \ldots \mathrm{O}=2.74-2.78 \AA\right)$, the two benzylic oxygens $\left(\mathrm{Ba}^{2+} \ldots \mathrm{O}=2.96-3.26 \AA\right)$, and the three alternating methoxy oxygens $\left(\mathrm{Ba}^{2+} \ldots \mathrm{O}=2.88-2.89 \AA\right)$. Besides these seven oxygens of the macrocycle two molecules of $\mathrm{DMF}^{31}$ are coordi-

(32) Brennan, T. D.; Scheidt, W. R. Acta Crystallogr., Sect. C 1988, 44, 478. 
Table IV. Final Positional Parameters and Equivalent Isotropic Thermal Parameters for $10 \cdot \mathrm{H}_{2} \mathrm{O}^{a}$

\begin{tabular}{|c|c|c|c|c|c|c|c|c|c|}
\hline atom & $x$ & $y$ & $z$ & $B, \AA^{2}$ & atom & $x$ & $y$ & $z$ & $B, \AA^{2}$ \\
\hline $\mathrm{Ba}$ & $0.83866(5)$ & $0.09288(4)$ & $0.78876(3)$ & $3.481(8)$ & $\mathrm{C} 24$ & $0.7945(8)$ & $0.3474(7)$ & $0.4896(5)$ & $4.5(2)$ \\
\hline $\mathrm{Ni}$ & $0.69562(9)$ & -0.02840 & $0.57873(5)$ & $3.43(2)$ & $\mathrm{C} 25$ & $0.830(1)$ & $0.4451(7)$ & $0.5416(6)$ & $5.7(2)$ \\
\hline S50 & $0.8722(3)$ & $0.0951(2)$ & $1.2329(2)$ & $6.10(6)$ & $\mathrm{C} 26$ & $0.826(1)$ & $0.4362(7)$ & $0.6211(6)$ & $5.2(2)$ \\
\hline S60 & $0.2407(3)$ & $0.2698(2)$ & $0.8534(2)$ & $7.03(7)$ & $\mathrm{C} 27$ & $0.7281(8)$ & $0.1354(7)$ & $0.4636(5)$ & $4.1(2)$ \\
\hline F55 & $0.852(1)$ & $0.3002(8)$ & $1.2958(5)$ & $15.7(3)$ & C29 & $0.6616(8)$ & $-0.0678(7)$ & $0.4143(5)$ & $4.4(2)$ \\
\hline F56 & $1.0679(9)$ & $0.2816(6)$ & $1.2808(9)$ & $19.7(4)$ & $\mathrm{C} 30$ & $0.7142(7)$ & $-0.1775(6)$ & 0.4497 (4) & $3.7(1)$ \\
\hline F57 & 0.890 (1) & $0.3030(6)$ & $1.1891(5)$ & $15.7(4)$ & C31 & $0.8872(8)$ & $-0.1709(7)$ & $0.4679(5)$ & $4.9(2)$ \\
\hline F65 & $0.151(1)$ & $0.4331(7)$ & $0.9312(6)$ & $17.0(3)$ & C32 & 0.636 (1) & $-0.2839(8)$ & $0.3917(5)$ & $5.6(2)$ \\
\hline F66 & $0.356(2)$ & $0.405(1)$ & $0.9754(7)$ & $23.9(5)$ & C54 & $0.927(1)$ & $0.2508(9)$ & $1.2521(7)$ & $6.9(3)$ \\
\hline F67 & $0.311(2)$ & $0.4888(8)$ & $0.8787(7)$ & $24.1(5)$ & C64 & $0.263(1)$ & $0.407(1)$ & $0.9082(7)$ & $8.2(3)$ \\
\hline OW & $0.5333(6)$ & $0.1064(5)$ & $0.7807(4)$ & $5.4(1)$ & HO1 & $0.496(6)$ & $0.143(5)$ & 0.784 & $2(1)^{*}$ \\
\hline O5 & $0.6780(5)$ & $-0.0812(4)$ & $0.6783(3)$ & $4.1(1)$ & $\mathrm{H} 2$ & $0.603(6)$ & $-0.333(5)$ & $0.514(3)$ & $3(1)^{*}$ \\
\hline 010 & $0.6509(7)$ & -0.1118 & $0.8254(3)$ & $5.5(1)$ & $\mathrm{HO} 2$ & $0.58(2)$ & 0.08 (1) & $0.856(9)$ & $18(6)^{*}$ \\
\hline 013 & $0.8072(9)$ & $0.0554(7)$ & $0.9423(4)$ & $8.0(2)$ & $\mathrm{H} 7$ & $0.559(8)$ & $-0.305(6)$ & $0.846(4)$ & $5(2)^{*}$ \\
\hline 016 & $0.8232(7)$ & $0.2892(5)$ & 0.8888 & $5.8(1)$ & $\mathrm{H} 8$ & $0.43(1)$ & $-0.493(8)$ & $0.738(5)$ & $7(2)^{*}$ \\
\hline O19 & $0.7796(6)$ & $0.3106(4)$ & $0.7269(3)$ & $4.6(1)$ & $\mathrm{H} 9$ & $0.502(7)$ & $-0.454(6)$ & $0.613(4)$ & $4(1)^{*}$ \\
\hline $\mathrm{O} 22$ & $0.7363(5)$ & $0.1202(4)$ & $0.6304(3)$ & $3.9(1)$ & H11B & 0.52 (1) & -0.18 (1) & $0.880(6)$ & $11(3)^{*}$ \\
\hline O51 & $0.931(1)$ & $0.0483(9)$ & $1.3069(6)$ & $14.0(3)$ & HIIA & $0.70(2)$ & $-0.19(1)$ & $0.925(8)$ & $15(4)^{*}$ \\
\hline O52 & $0.7202(9)$ & $0.0799(8)$ & $1.2072(9)$ & $15.5(5)$ & $\mathrm{H} 12 \mathrm{~A}$ & $0.678(8)$ & $-0.008(6)$ & $1.012(4)$ & $5(2)^{*}$ \\
\hline O53 & $0.9606(8)$ & $0.0672(6)$ & $1.1805(5)$ & $8.4(2)$ & $\mathrm{H} 12 \mathrm{~B}$ & 0.585 & 0.013 & 0.928 & $6.0^{*}$ \\
\hline O61 & $0.221(3)$ & 0.192 (1) & $0.9062(8)$ & $35(1)$ & $\mathrm{H} 14 \mathrm{~A}$ & $0.99(1)$ & $0.166(7)$ & $0.998(5)$ & $7(2)^{*}$ \\
\hline 062 & $0.109(1)$ & $0.2551(9)$ & $0.7998(7)$ & $16.8(3)$ & $\mathrm{H} 14 \mathrm{~B}$ & $0.85(1)$ & $0.153(9)$ & $1.059(6)$ & $9(3)^{*}$ \\
\hline O63 & $0.3636(9)$ & $0.2776(9)$ & $0.8183(7)$ & $14.2(3)$ & $\mathrm{H} 15 \mathrm{~B}$ & $0.843(9)$ & $0.323(7)$ & $1.010(5)$ & $6(2)^{*}$ \\
\hline $\mathrm{Nl}$ & $0.6652(6)$ & $-0.1765(5)$ & $0.5282(4)$ & $4.0(1)$ & H15A & 0.682 & 0.253 & 0.955 & $5.0^{*}$ \\
\hline $\mathrm{N} 28$ & $0.7023(6)$ & 0.0268 & $0.4784(3)$ & $3.6(1)$ & H17A & 0.78 & $0.46(1)$ & $0.887(8)$ & $13(4)^{*}$ \\
\hline $\mathrm{C} 2$ & $0.6169(8)$ & $-0.2727(6)$ & $0.5575(5)$ & $4.0(2)$ & $\mathrm{H} 17 \mathrm{~B}$ & 0.61 (1) & $0.382(8)$ & $0.828(5)$ & $8(3)^{*}$ \\
\hline $\mathrm{C} 3$ & $0.5866(8)$ & $-0.2841(6)$ & $0.6340(5)$ & $4.3(2)$ & $\mathrm{H} 18 \mathrm{~A}$ & 0.77 (1) & $0.461(9)$ & $0.752(6)$ & $10(3)^{*}$ \\
\hline $\mathrm{C} 4$ & $0.6231(7)$ & -0.1888 & $0.6925(5)$ & $3.9(2)$ & $\mathrm{H} 18 \mathrm{~B}$ & $0.94(1)$ & $0.436(8)$ & 0.799 (5) & $7(2)^{*}$ \\
\hline C6 & $0.6046(8)$ & $-0.2097(6)$ & $0.7692(5)$ & $4.7(2)$ & $\mathrm{H} 24$ & $0.787(8)$ & $0.346(6)$ & $0.431(4)$ & $5(2)^{*}$ \\
\hline $\mathrm{C} 7$ & $0.543(1)$ & $-0.3195(8)$ & $0.7883(6)$ & $5.9(2)$ & $\mathrm{H} 25$ & $0.87(1)$ & $0.56(1)$ & $0.525(7)$ & $13(4)^{*}$ \\
\hline $\mathrm{C} 8$ & $0.502(1)$ & $-0.4135(7)$ & $0.7287(6)$ & $5.8(2)$ & $\mathrm{H} 26$ & $0.87(1)$ & $0.490(7)$ & $0.661(5)$ & $7(2)^{*}$ \\
\hline C9 & $0.526(1)$ & $-0.3959(7)$ & $0.6539(6)$ & $5.3(2)$ & $\mathrm{H} 27$ & $0.74(1)$ & $0.164(8)$ & $0.415(6)$ & $8(3)^{*}$ \\
\hline C11 & $0.648(1)$ & $-0.1285(9)$ & $0.9071(6)$ & $7.2(3)$ & $\mathrm{H} 29 \mathrm{~B}$ & 0.75 (1) & $-0.043(8)$ & $0.370(5)$ & $7(2)^{*}$ \\
\hline $\mathrm{C} 12$ & $0.676(1)$ & $-0.014(1)$ & $0.9522(5)$ & $8.6(3)$ & $\mathrm{H} 29 \mathrm{~A}$ & $0.54(1)$ & $-0.112(8)$ & $0.364(5)$ & $8(3)^{*}$ \\
\hline $\mathrm{C} 14$ & 0.861 (1) & 0.165 (1) & $0.9935(6)$ & $8.3(3)$ & $\mathrm{H} 31 \mathrm{~A}$ & $0.94(1)$ & $-0.118(8)$ & $0.505(5)$ & $7(2)^{*}$ \\
\hline $\mathrm{C} 15$ & $0.790(1)$ & $0.262(1)$ & $0.9651(6)$ & $8.2(3)$ & $\mathrm{H} 31 \mathrm{C}$ & $0.927(9)$ & $-0.157(7)$ & $0.410(5)$ & $6(2)^{*}$ \\
\hline C17 & 0.756 (1) & $0.3823(8)$ & $0.8539(6)$ & $6.3(2)$ & $\mathrm{H} 31 \mathrm{~B}$ & $0.901(8)$ & $-0.241(6)$ & $0.501(4)$ & $5(2)^{*}$ \\
\hline $\mathrm{C} 18$ & $0.815(1)$ & $0.4129(7)$ & $0.7832(5)$ & $5.2(2)$ & $\mathrm{H} 32 \mathrm{~A}$ & $0.517(9)$ & $-0.301(7)$ & $0.383(5)$ & $6(2)^{*}$ \\
\hline $\mathrm{C} 20$ & $0.7906(8)$ & $0.3276(6)$ & $0.6490(5)$ & $4.0(2)$ & $\mathrm{H} 32 \mathrm{C}$ & 0.686 & -0.346 & 0.413 & $6.0^{*}$ \\
\hline C21 & $0.7620(7)$ & $0.2250(6)$ & $0.5990(4)$ & $3.6(1)$ & H32B & $0.641(9)$ & $-0.265(7)$ & $0.336(5)$ & $7(2)^{*}$ \\
\hline $\mathrm{C} 23$ & $0.7598(7)$ & $0.2358(6)$ & $0.5181(5)$ & $3.9(2)$ & & & & & \\
\hline
\end{tabular}

${ }^{a}$ See footnote $a$ of Table III.

nated to the barium at the same side as the DMF that is coordinated to the zinc $\left(\mathrm{Ba}^{2+} \ldots \mathrm{O}=\mathrm{C}=2.70-2.72 \AA\right)$. The tenth coordination site is occupied by a triflate anion $\left(\mathrm{Ba}^{2+} \ldots \mathrm{O}=2.59\right.$ $\AA$ ). The distance between the two metal ions is $3.63 \AA$.

Electrochemistry. As mentioned in the Introduction, a major objective of the work described in this paper was to study the effect of the hard cation in the macrocyclic cavity on the (redox) properties of the coordinated soft transition-metal cation. In order to evaluate this effect, a number of heterodinuclear complexes and mono-transition-metal complexes were investigated by sampled dc polarography in DMSO with $\mathrm{Et}_{4} \mathrm{~N}^{+} \mathrm{ClO}_{4}^{-}$as supporting electrolyte. The polarograms in the range of -0.2 to $-2 \mathrm{~V}$ were recorded and evaluated by a computerized method described by Zollinger et al., ${ }^{33}$ and the results are given in Table VII. In addition the nickel/barium complex 10, the copper/barium complex 12, the nickel complex 15, and the zinc/barium complex 25 were also studied with cyclic voltammetry, in DMSO also with $\mathrm{Et}_{4} \mathrm{~N}^{+} \mathrm{ClO}_{4}^{-}$as supporting electrolyte.

The slopes of the log plots [ $\log \left(i_{\mathrm{d}}-i\right) / i$ vs $\left.\mathrm{V}\right]$ for the monocopper complexes 16, 17, and 27 are in agreement with a reversible one-electron reduction, as has also been reported for similar copper complexes with ligands derived from aromatic or aliphatic diamino compounds, and $o$-hydroxybenzaldehyde or $o$-hydroxynaphthaldehyde at a gold electrode in DMF. ${ }^{34}$ Enlargement of the ring size shows a small anodic shift of the $E_{1 / 2}$ values for the reduction

(33) Zollinger, D. P.; Bos, M.; van Veen-Blaauw, A. M. W.; van der Linden, W. E. Anal. Chim. Acta 1985, 167, 89.

(34) Rohrbach, D. F.; Heineman, W. R.; Deutsch, E. Inorg. Chem. 1979, 18,2536 . of 16 and $17(-1.332$ and $-1.265 \mathrm{~V}$, respectively). Reduction of 27 takes place at a more anodic $E_{1 / 2}(-1.008 \mathrm{~V})$. This may be due to the aromatic ring connecting the two imine bonds or to a combination of this aromatic ring and the more rigid polyether cavity.

When alkali- or alkaline-earth-metal cations are complexed in these dinucleating ligands, an anodic shift of the half-wave potential varying from $9 \mathrm{mV}$ for 17 with $\mathrm{K}^{+}$up to $213 \mathrm{mV}$ for 16 with $\mathrm{Ba}^{2+}$ is observed (Table VIII). The presence of $\mathrm{K}^{+}$did not affect the characteristics of compound 27 . For the cations $\mathrm{Ba}^{2+}$, $\mathrm{K}^{+}$, and $\mathrm{Na}^{+}$the results indicate a reversible one-electron reduction, but for $\mathrm{Li}^{+}$the reduction is far from reversible. In all cases the bivalent $\mathrm{Ba}^{2+}$ gave the largest shift in the half-wave potential. It is known from the literature that the reduction can be shifted anodically or cathodically by introducing substituents in (copper) complexing ligands. ${ }^{35}$ Cathodic shifts (up to $690 \mathrm{mV}$ ) upon complexation of cations $\left(\mathrm{Ba}^{2+}, \mathrm{Ca}^{2+}, \mathrm{Mg}^{2+}, \mathrm{Zn}^{2+}\right)$ and at the same time deprotonation of two carboxylic groups in monocopper complexes of a noncyclic dinucleating ligand have been reported by Kanda et al. ${ }^{2 a}$ Anodic shifts of molybdenum compounds that contain crown ether moieties with complexed hard cations have been reported by Beer et al. ${ }^{36}$ Upon the addition of 1 equiv of $\mathrm{Ba}\left(\mathrm{ClO}_{4}\right)_{2}$ to the monocopper complexes 16,17 , and 27, respectively, the characteristics of the heterodinuclear complexes 12, 13, and 24 were observed and the addition of more

(35) Araya, L. M.; Vargas, J. A.; Costamagna, J. A. Transition Met. Chem. 1986, 11,312

(36) Beer, P. D.; Jones, C. J.; McCleverty, J. A.; Salam, S. S. J. Inclusion Phenom. 1987, 5, 521 . 
Table V. Final Positional Parameters and Equivalent Isotropic Thermal Parameters for $10 \cdot \mathrm{Na}^{+} \mathrm{Pic}^{-a}$

\begin{tabular}{|c|c|c|c|c|c|c|c|c|c|}
\hline atom & $x$ & $y$ & $z$ & $B, \AA^{2}$ & atom & $x$ & $y$ & $z$ & $B, \AA^{2}$ \\
\hline $\mathrm{Ni}$ & $0.7264(2)$ & $0.3074(2)$ & $0.1204(2)$ & $4.43(7)$ & C30 & $0.541(2)$ & $0.380(2)$ & $0.099(2)$ & $14(1)$ \\
\hline $\mathrm{Na}$ & $0.7276(7)$ & $0.0597(6)$ & $0.1488(5)$ & $4.6(2)$ & C32 & $0.667(2)$ & $0.493(2)$ & $0.074(4)$ & $25(2)$ \\
\hline $\mathrm{O} 12$ & $0.811(1)$ & $0.217(1)$ & 0.135 (1) & $4.8(4)^{*}$ & $\mathrm{C} 40$ & $0.240(1)$ & $0.633(1)$ & $0.154(1)$ & $3.5(5)^{*}$ \\
\hline 013 & $0.905(1)$ & 0.068 & $0.1470(9)$ & $4.6(4)^{*}$ & C41 & $0.155(1)$ & $0.677(1)$ & $0.133(1)$ & $2.3(4)^{*}$ \\
\hline 016 & $0.824(1)$ & $-0.096(1)$ & 0.151 (1) & $5.1(4)^{*}$ & $\mathrm{C} 42$ & $0.153(1)$ & $0.773(1)$ & 0.107 (1) & $3.3(5)^{*}$ \\
\hline 019 & $0.634(1)$ & $-0.095(1)$ & $0.140(1)$ & $7.1(5)^{*}$ & $\mathrm{C} 43$ & $0.228(1)$ & $0.815(1)$ & $0.090(1)$ & $3.2(5)^{*}$ \\
\hline $\mathrm{O} 22$ & $0.552(1)$ & $0.063(1)$ & $0.135(1)$ & $9.4(6)^{*}$ & C44 & $0.311(1)$ & $0.770(1)$ & 0.099 (1) & $3.6(5)^{*}$ \\
\hline $\mathrm{O} 29$ & $0.6447(8)$ & $0.2167(8)$ & $0.1304(8)$ & $2.6(3)^{*}$ & $\mathrm{C} 45$ & $0.318(1)$ & $0.687(2)$ & $0.125(1)$ & $3.8(5)^{*}$ \\
\hline O51 & 0.290 (1) & $0.942(1)$ & 0.028 (1) & $5.1(4)^{*}$ & $\mathrm{H} 2 \mathrm{~A}$ & 0.732 & 0.584 & 0.215 & $5.0^{*}$ \\
\hline O52 & 0.151 (1) & $0.952(1)$ & $0.060(1)$ & $7.3(5)^{*}$ & $\mathrm{H} 2 \mathrm{~B}$ & 0.729 & 0.481 & 0.241 & $5.0^{*}$ \\
\hline O54 & 0.008 (1) & 0.678 (1) & 0.171 (1) & $5.8(4)^{*}$ & $\mathrm{H} 2 \mathrm{C}$ & 0.822 & 0.529 & 0.236 & $5.0^{*}$ \\
\hline O55 & $0.060(1)$ & $0.556(1)$ & $0.119(1)$ & $6.0(4)^{*}$ & $\mathrm{H} 3 \mathrm{~A}$ & 0.768 & 0.624 & 0.058 & $5.0^{*}$ \\
\hline O56 & $0.243(1)$ & $0.556(1)$ & $0.1886(9)$ & $4.3(4)^{*}$ & $\mathrm{H} 3 \mathrm{~B}$ & 0.860 & 0.572 & 0.076 & $5.0^{*}$ \\
\hline O57 & $0.415(2)$ & $0.563(1)$ & $0.146(2)$ & $-17(1)$ & $\mathrm{H} 3 \mathrm{C}$ & 0.792 & 0.547 & -0.008 & $5.0^{*}$ \\
\hline 058 & $0.472(1)$ & $0.688(2)$ & $0.134(1)$ & $9.2(5)^{*}$ & $\mathrm{H} 5$ & 0.913 & 0.451 & 0.082 & $5.0^{*}$ \\
\hline N4 & $0.802(1)$ & $0.401(1)$ & $0.106(1)$ & $4.0(4)^{*}$ & $\mathrm{H} 7$ & 1.055 & 0.364 & 0.062 & $5.0^{*}$ \\
\hline N31 & $0.636(2)$ & $0.397(2)$ & $0.101(2)$ & $9.4(7)^{*}$ & $\mathrm{H} 8$ & 1.132 & 0.238 & 0.057 & $5.0^{*}$ \\
\hline N50 & 0.224 (1) & $0.910(1)$ & 0.058 & $5.6(5)^{*}$ & $\mathrm{H} 9$ & 1.074 & 0.090 & 0.084 & $5.0^{*}$ \\
\hline N53 & $0.070(1)$ & $0.634(1)$ & $0.144(1)$ & $4.0(4)^{*}$ & $\mathrm{H} 14 \mathrm{~A}$ & 0.966 & -0.038 & 0.093 & $5.0^{*}$ \\
\hline N56 & $0.405(1)$ & $0.640(2)$ & $0.139(1)$ & $6.5(6)^{*}$ & $\mathrm{H} 14 \mathrm{~B}$ & 1.017 & -0.006 & 0.185 & $5.0^{*}$ \\
\hline $\mathrm{Cl}$ & $0.756(2)$ & $0.495(2)$ & $0.110(2)$ & $5.8(7)^{*}$ & H15A & 0.943 & -0.142 & 0.203 & $5.0^{*}$ \\
\hline $\mathrm{C} 2$ & $0.761(2)$ & $0.526(2)$ & $0.210(2)$ & $11(1)^{*}$ & H15B & 0.903 & -0.063 & 0.256 & $5.0^{*}$ \\
\hline $\mathrm{C} 3$ & $0.797(2)$ & $0.566(3)$ & $0.054(2)$ & $18(2)$ & $\mathrm{H} 17 \mathrm{~A}$ & 0.809 & -0.219 & 0.195 & $5.0^{*}$ \\
\hline C5 & $0.884(2)$ & $0.395(2)$ & $0.094(2)$ & $5.7(6)^{*}$ & H17B & 0.761 & -0.143 & 0.247 & $5.0^{*}$ \\
\hline C6 & $0.936(1)$ & $0.317(2)$ & $0.097(1)$ & $4.1(5)^{*}$ & $\mathrm{H} 18 \mathrm{~A}$ & 0.699 & -0.187 & 0.070 & $5.0^{*}$ \\
\hline C7 & $1.025(1)$ & $0.309(2)$ & $0.076(1)$ & $5.0(6)^{*}$ & $\mathrm{H} 18 \mathrm{~B}$ & 0.655 & -0.231 & 0.151 & $5.0^{*}$ \\
\hline $\mathrm{C} 8$ & $1.071(2)$ & $0.233(2)$ & $0.072(2)$ & $6.2(7)^{*}$ & $\mathrm{H} 20 \mathrm{C}$ & 0.545 & -0.154 & 0.192 & $5.0^{*}$ \\
\hline C9 & $1.040(2)$ & $0.145(2)$ & $0.090(1)$ & $5.4(6)^{*}$ & $\mathrm{H} 20 \mathrm{D}$ & 0.595 & -0.077 & 0.250 & $5.0^{*}$ \\
\hline $\mathrm{ClO}$ & $0.948(2)$ & $0.140(2)$ & $0.117(2)$ & $5.0(6)^{*}$ & $\mathrm{H} 20 \mathrm{~A}$ & 0.577 & -0.071 & 0.028 & $5.0^{*}$ \\
\hline $\mathrm{C} 11$ & $0.902(1)$ & $0.221(1)$ & $0.118(1)$ & $3.9(5)^{*}$ & $\mathrm{H} 20 \mathrm{~B}$ & 0.541 & -0.156 & 0.077 & $5.0^{*}$ \\
\hline $\mathrm{C} 14$ & $0.958(1)$ & -0.017 (1) & 0.153 (1) & $3.9(5)^{*}$ & $\mathrm{H} 21 \mathrm{C}$ & 0.470 & -0.041 & 0.091 & $5.0^{*}$ \\
\hline $\mathrm{C} 15$ & $0.911(2)$ & $-0.086(2)$ & $0.197(2)$ & $6.6(7)^{*}$ & $\mathrm{H} 21 \mathrm{D}$ & 0.455 & -0.010 & 0.190 & $5.0^{*}$ \\
\hline $\mathrm{C} 17$ & $0.775(2)$ & $-0.164(2)$ & $0.188(2)$ & $10(1)^{*}$ & $\mathrm{H} 21 \mathrm{~A}$ & 0.438 & -0.014 & 0.122 & $5.0^{*}$ \\
\hline $\mathrm{C} 18$ & $0.687(2)$ & $-0.179(2)$ & $0.132(2)$ & $8.4(8)^{*}$ & $\mathrm{H} 21 \mathrm{~B}$ & 0.499 & -0.039 & 0.210 & $5.0^{*}$ \\
\hline C20A & $0.567(3)$ & $-0.097(3)$ & $0.086(3)$ & $5(1)^{*}$ & $\mathrm{H} 24$ & 0.372 & 0.086 & 0.129 & $5.0^{*}$ \\
\hline $\mathrm{C} 20 \mathrm{~B}$ & $0.571(4)$ & -0.095 & $0.190(4)$ & $9(2)^{*}$ & $\mathrm{H} 25$ & 0.299 & 0.222 & 0.128 & $5.0^{*}$ \\
\hline C21 & $0.499(2)$ & $-0.021(2)$ & $0.148(3)$ & $18(2)$ & $\mathrm{H} 26$ & 0.378 & 0.362 & 0.120 & $5.0^{*}$ \\
\hline $\mathrm{C} 23$ & $0.498(2)$ & $0.149(2)$ & $0.131(2)$ & $6.5(7)^{*}$ & $\mathrm{H} 30$ & 0.499 & 0.429 & 0.086 & $5.0^{*}$ \\
\hline $\mathrm{C} 24$ & $0.404(2)$ & $0.144(2)$ & $0.130(2)$ & $9.2(9)^{*}$ & H32B & 0.631 & 0.542 & 0.093 & $5.0^{*}$ \\
\hline $\mathrm{C} 25$ & $0.363(2)$ & $0.222(2)$ & $0.127(2)$ & $8.0(8)^{*}$ & $\mathrm{H} 32 \mathrm{~A}$ & 0.664 & 0.493 & 0.009 & $5.0^{*}$ \\
\hline $\mathrm{C} 26$ & $0.409(2)$ & $0.305(2)$ & $0.119(2)$ & $7.0(7)^{*}$ & $\mathrm{H} 42$ & 0.097 & 0.806 & 0.103 & $5.0^{*}$ \\
\hline $\mathrm{C} 27$ & $0.511(2)$ & $0.301(2)$ & $0.118(2)$ & $7.0(7)^{*}$ & $\mathrm{H} 44$ & 0.364 & 0.802 & 0.084 & $5.0^{*}$ \\
\hline $\mathrm{C} 28$ & 0.555 (1) & $0.222(1)$ & 0.131 (1) & $4.0(5)^{*}$ & & & & & \\
\hline
\end{tabular}

${ }^{a}$ See footnote $a$ of Table III.

$\mathrm{Ba}\left(\mathrm{ClO}_{4}\right)_{2}$ did not affect these values. This indicates a stoichiometric $1: 1$ reaction between $\mathrm{Ba}^{2+}$ and the ligands and large association constants of these complexes. ${ }^{37}$ The same observations were made for compound 16 with $\mathrm{K}^{+}$and $\mathrm{Na}^{+}$as guest cations. For compound 16 with $\mathrm{Li}^{+}$the shift of the half-wave potential does not seem to reach a maximum even with 5 equiv of $\mathrm{Li}^{+}$ present. From this it may be concluded that the association constant of the complex of $\mathbf{1 6}$ and $\mathrm{Li}^{+}$is much smaller than those of the $\mathrm{Na}^{+}, \mathrm{K}^{+}$, and $\mathrm{Ba}^{2+}$ complexes. In the case of 17 the association constant of the $\mathrm{Ba}^{2+}$ and $\mathrm{K}^{+}$complexes will be larger than those of the $\mathrm{Na}^{+}$and $\mathrm{Li}^{+}$complexes. The reduction of the zinc/barium complex 25 takes place at the most cathodic halfwave potential and is most likely an irreversible two-electron reduction. Cyclic voltammetry revealed an EC mechanism, i.e. first an electrochemical step followed by a chemical reaction (probably decomposition). This conclusion is based on the fact that when the scan rate is increased $(0.5,1,2, \ldots, 6 \mathrm{~V} / \mathrm{s})$ the peak surface of the oxidation sweep becomes larger and larger relative to the peak surface of the reduction sweep: $45 \%$ at $0.5 \mathrm{~V} / \mathrm{s}$ up to $90 \%$ at $6 \mathrm{~V} / \mathrm{s}$.

The electrochemical behavior of the copper/barium complex 12 was also studied by cyclic voltammetry. At scan rates from 1 to $6 \mathrm{~V} / \mathrm{s}$ the cyclic voltammograms showed one reduction and

(37) The association constants for the alkali-metal and alkaline-earth-metal cations could not be determined in this system because the reduction of these metal ions takes place at a too cathodic potential and is often hidden under the reduction of the electrolyte, water, and solvent. one oxidation between -0.2 and $-2 \mathrm{~V}$. The peak separation $\left(E_{\mathrm{pa}}\right.$ $\left.-E_{\mathrm{pc}}\right)$ was not sensitive to the scan rate $\left(\Delta E_{\mathrm{p}}=88-98 \mathrm{mV}\right)$, which indicates an irreversible electron-transfer reaction. At these scan rates the peak surface of the cathodic and anodic sweep were equal within $10 \%$, which suggests a chemically reversible reduction. Therefore, we conclude that the $\mathrm{Cu}^{\mathrm{I}}$ species are stable in a DMSO solution in the absence of oxygen.

The dinuclear nickel/barium complexes 10, 23, and 28 exhibited one wave, and these reductions likely proceed via a reversible one-electron transfer. The polarograms of the mononickel complexes 14, 15, 26, and 29 are more complex and showed two waves. Adding 1 equiv of $\mathrm{Ba}\left(\mathrm{ClO}_{4}\right)_{2}$ to solutions of $14,15,26$, and 29 gave the characteristics of the complexes 10, 11, 23, and 28, respectively. Therefore, the two recorded waves correspond with the mononickel complexes. The addition of more $\mathrm{Ba}\left(\mathrm{ClO}_{4}\right)_{2}$ did not affect these values. It is most likely that the reduction of the mononickel complexes is a one-electron transfer as has been reported for nickel complexes ${ }^{38}$ with the same coordination as in this report at a platinum electrode in DMSO, DMF, and $\mathrm{CH}_{3} \mathrm{CN}$, and the first wave might be due to adsorption ${ }^{39}$ (vide infra). In the cases of 14, 15, and 29 the electron density in the reduced form is probably concentrated at the nickel cation, and in the case of 26 the electron is delocalized over the ligand as is shown by

(38) Gosden, C.; Kerr, J. B.; Pletcher, D.; Rosas, R. J. Electroanal. Chem. Interfacial Electrochem. 1981, il7, 101.

(39) Heyrovski, J.; Kuta, J. Grundlagen der Polarographie; AkademieVerlag: Berlin, 1965. 
a

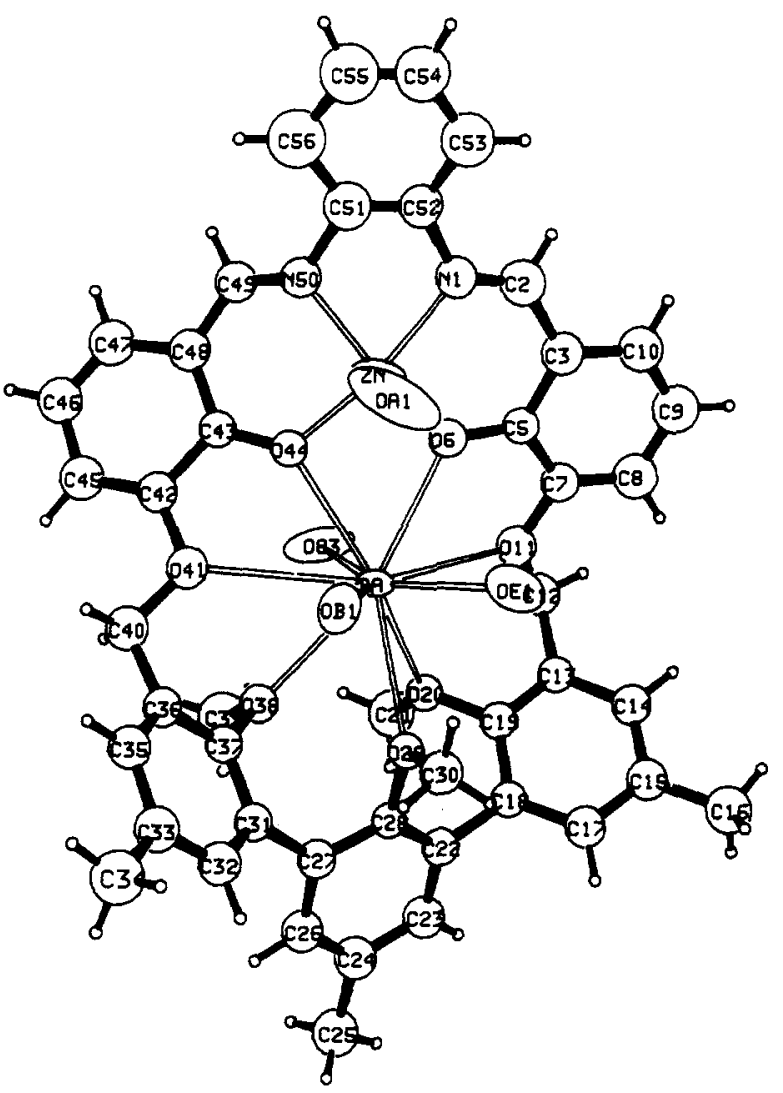

b

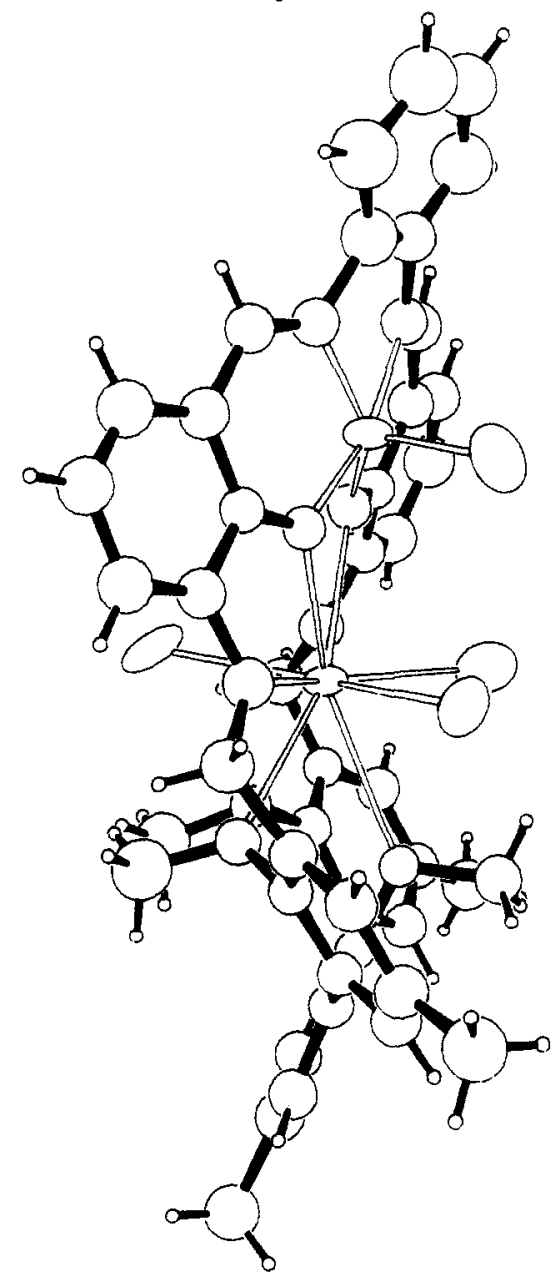

Figure 5. Front (a) and side (b) views of 25.3DMF. Only the coordinated oxygens of the three DMF molecules and of the coordinated anion are shown for clarity. Thermal ellipsoids of non-hydrogen atoms are shown at the $50 \%$ probability level.
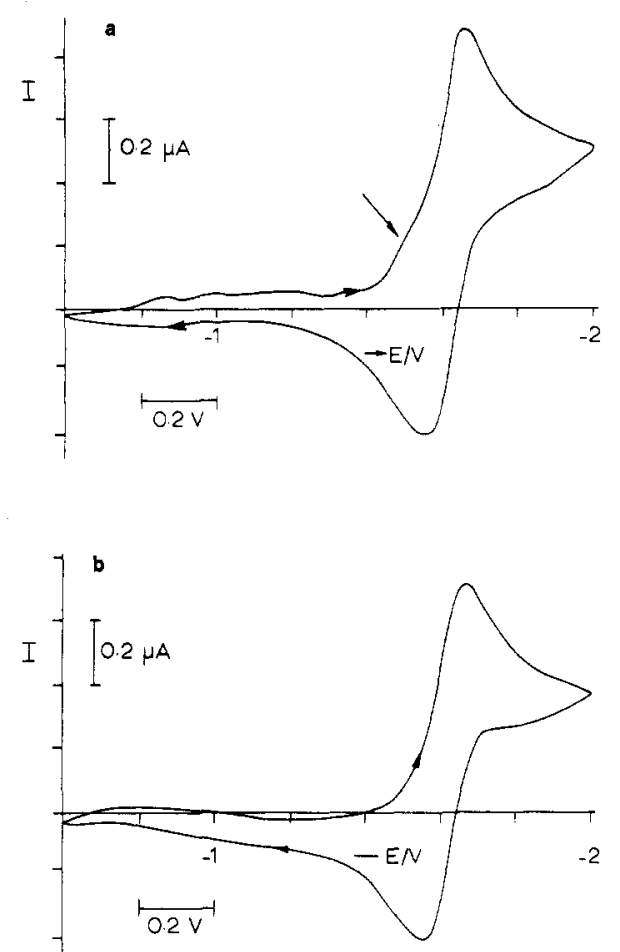

Figure 6. Cyclic voltammograms of 15 in DMSO at a scan rate of $1 \mathrm{~V} / \mathrm{s}$ after the first scan (a) and after the fifth scan (b).

Gosden et al..$^{38}$ with ESR measurements of $\mathrm{Ni}^{\mathrm{I}}$ species with the same coordination.

The nickel/barium complex $\mathbf{1 0}$ and the mononuclear nickel complex 15 were also investigated in more detail by cyclic voltammetry. At scan rates of 0.5 to $6 \mathrm{~V} / \mathrm{s}$ the dinuclear complex 10 showed one reduction and one oxidation between -0.2 and -2 $\mathrm{V}$. The peak separation $\left(E_{\mathrm{pa}}-E_{\mathrm{pc}}\right)$ was slightly sensitive to the scan rate; $80 \mathrm{mV}$ at $0.5 \mathrm{~V} / \mathrm{s}$ down to $69 \mathrm{mV}$ at $6 \mathrm{~V} / \mathrm{s}$. At these scan rates the peak surfaces of reduction and oxidation sweeps were equal. When we applied five subsequent scans at scan rates of 0.5 and $6 \mathrm{~V} / \mathrm{s}$, the cyclic voltammograms were completely identical. The generated $\mathrm{Ni}^{\mathrm{I}}$ species is thus chemically stable in DMSO in the absence of oxygen. When we applied one scan for the nickel complex 15 at a scan rate of $1 \mathrm{~V} / \mathrm{s}$, a "prewave" in the cathodic sweep was observed (Figure 6a). When five subsequent scans were applied, the "prewave" disappeared after the first scan (Figure 6b). When after 1 min five scans were again applied, we found the same pattern, and this indicates that the prewave is a result of a slow adsorption process. The reduction of $\mathbf{1 5}$ is chemically a reversible process but electrochemically an irreversible process at scan rates of $50 \mathrm{mV} / \mathrm{s}$ to $2 \mathrm{~V} / \mathrm{s}$. The $i_{p c}$ and $i_{\mathrm{pa}}$ values for 10 and 15 are linear to the square root of the scan rate, indicating a diffusion-controlled mechanism.

When we compare the nickel/barium complexes 10 and 11 with 28 , we found a relatively large effect on the half-wave potential going from flexible crown ether ligands in $\mathbf{1 0}$ and $11(-1.338$ and $-1.359 \mathrm{~V}$, respectively) to $\mathbf{2 8}$, which has a much more rigid terphenyl-containing cavity $(-1.237 \mathrm{~V})$. The stabilizing effect of the $\mathrm{Ni}^{1}$ complex by an aromatic vs an aliphatic diamino moiety is obvious when we compare the reduction of 23 and $28(-1.018$ vs $-1.237 \mathrm{~V}$ ). When $\mathrm{Li}^{+}, \mathrm{Na}^{+}$, or $\mathrm{K}^{+}$was added to 14 and 15 , two waves were recorded with characteristics other than those of the cation-free nickel complexes, showing that the alkali-metal cations are complexed in the crown ether moiety. The addition of 1 equiv of $\mathrm{K}^{+}$to 26 and 29 gave a reversible one-electron reduction, excess of $\mathrm{K}^{+}$did not change the electrochemical characteristics.

We assume that the effect of the hard cations $\mathrm{Ba}^{2+}, \mathrm{K}^{+}, \mathrm{Na}^{+}$, and $\mathrm{Li}^{+}$, complexed in the polyether cavity, is the reduction of electron density at the phenolic oxygen atoms and this reduces the electron-donating ability toward the transition-metal ion. Consequently the transition-metal ion is more positive and 
Table VI. Final Positional Parameters and Equivalent Isotropic Thermal Parameters for 25.3DMFa

\begin{tabular}{|c|c|c|c|c|c|c|c|c|c|}
\hline atom & $x$ & $y$ & $z$ & $B, \AA^{2}$ & atom & $x$ & $y$ & $z$ & $B, \AA^{2}$ \\
\hline $\mathrm{Ba}$ & $0.24429(3)$ & $-0.00301(3)$ & $-0.19589(4)$ & $2.87(2)$ & $\mathrm{C} 30$ & $0.1560(6)$ & $0.0267(6)$ & $-0.3851(6)$ & $4.0(3)^{*}$ \\
\hline $\mathrm{Zn}$ & $0.37339(7)$ & $-0.09191(7)$ & $-0.10754(8)$ & $4.10(4)$ & C31 & $0.0378(5)$ & $-0.0036(5)$ & $-0.3548(5)$ & $3.2(2)^{*}$ \\
\hline S80 & $0.7111(2)$ & $0.0223(2)$ & $0.5081(2)$ & $7.6(2)$ & $\mathrm{C} 32$ & $-0.0010(6)$ & $-0.0327(6)$ & $-0.4107(7)$ & $4.0(3)^{*}$ \\
\hline F85 & $0.7414(6)$ & $0.0347(6)$ & $0.4143(5)$ & $11.7(4)$ & C33 & $-0.0080(6)$ & $-0.0833(6)$ & $-0.4042(7)$ & $4.4(3)^{*}$ \\
\hline F86 & $0.3071(5)$ & $0.4887(6)$ & $0.5027(5)$ & $15.9(5)$ & C34 & $-0.0505(7)$ & $-0.1161(6)$ & $-0.4688(7)$ & $6.0(4)^{*}$ \\
\hline F87 & $0.6969(5)$ & $0.4293(6)$ & $0.4927(5)$ & $13.0(4)$ & C35 & $0.0268(6)$ & $-0.1075(6)$ & $-0.3412(6)$ & $4.0(3)^{*}$ \\
\hline F90 & $0.7211(5)$ & $0.2645(6)$ & $0.4888(5)$ & $11.6(4)$ & C36 & $0.0651(6)$ & $-0.0800(5)$ & $-0.2847(6)$ & $3.5(3)^{*}$ \\
\hline F100 & $0.4950(3)$ & $0.2352(3)$ & 0.7021 (4) & $5.5(2)$ & C37 & $0.0712(5)$ & $-0.0281(5)$ & $-0.2921(6)$ & $3.1(3)^{*}$ \\
\hline OE1 & $0.3031(5)$ & $0.0145(4)$ & $0.7293(5)$ & $6.2(3)$ & C39 & $0.0864(7)$ & $0.0214(7)$ & $-0.1962(7)$ & $5.9(4)^{*}$ \\
\hline OB 1 & $0.7060(4)$ & $0.4261(4)$ & $0.7046(4)$ & $4.4(3)$ & $\mathrm{C} 40$ & $0.0957(6)$ & $-0.1051(6)$ & $-0.2158(6)$ & $4.3(3)^{*}$ \\
\hline OAl & $0.3565(5)$ & $0.1255(6)$ & $0.3033(6)$ & $10.4(4)$ & $\mathrm{C} 42$ & $0.1954(6)$ & $-0.1235(5)$ & $-0.1208(6)$ & $3.8(3)^{*}$ \\
\hline O6 & $0.3715(4)$ & $-0.0176(3)$ & $-0.1169(4)$ & $3.6(2)^{*}$ & $\mathrm{C} 43$ & $0.2634(5)$ & $-0.1226(5)$ & $-0.0965(6)$ & $3.2(3)^{*}$ \\
\hline O11 & $0.3416(4)$ & $0.0776(4)$ & $-0.1444(4)$ & $4.0(2)^{*}$ & C45 & $0.1675(7)$ & $-0.1493(6)$ & $-0.0890(7)$ & $4.6(3)^{*}$ \\
\hline $\mathrm{O} 20$ & $0.2033(3)$ & $0.1013(3)$ & $-0.2017(4)$ & $3.3(2)^{*}$ & $\mathrm{C} 46$ & $0.2061(6)$ & $-0.1769(6)$ & $-0.0292(7)$ & $4.9(4)^{*}$ \\
\hline $\mathrm{O} 29$ & $0.1541(3)$ & $0.0421(3)$ & $-0.3238(4)$ & $2.9(2)^{*}$ & $\mathrm{C} 47$ & $0.2675(6)$ & $-0.1784(6)$ & $-0.0069(7)$ & $5.0(4)^{*}$ \\
\hline $\mathrm{O} 38$ & $0.1126(3)$ & $0.0010(4)$ & $-0.2349(4)$ & $3.5(2)^{*}$ & $\mathrm{C} 48$ & $0.3000(6)$ & $-0.1542(6)$ & $-0.0373(7)$ & $4.2(3)^{*}$ \\
\hline O41 & $0.1619(4)$ & $-0.0958(4)$ & -0.1818 & $4.0(2)^{*}$ & C49 & $0.3640(6)$ & $-0.1658(5)$ & $-0.0169(7)$ & $4.0(3)^{*}$ \\
\hline $\mathrm{O} 44$ & $0.2849(3)$ & $-0.0954(3)$ & -0.1279 & $3.1(2)^{*}$ & C51 & $0.4601(7)$ & $-0.1691(6)$ & $-0.0244(8)$ & $5.5(4)^{*}$ \\
\hline O81 & $0.3174(6)$ & $-0.9727(5)$ & $0.5215(7)$ & $10.1(5)$ & C52 & $0.4954(6)$ & $-0.1407(6)$ & $-0.0357(7)$ & $4.4(3)^{*}$ \\
\hline O82 & $0.6726(6)$ & $0.0653(5)$ & $0.4798(7)$ & $10.6(5)$ & C53 & $0.5546(8)$ & -0.1559 (7) & $-0.0238(8)$ & $6.7(5)^{*}$ \\
\hline 083 & $0.7461(6)$ & $0.4799(5)$ & $0.4173(5)$ & $7.8(4)$ & C54 & $0.5700(8)$ & $-0.2067(7)$ & $-0.0001(8)$ & $6.8(5)^{*}$ \\
\hline O91 & $0.6710(6)$ & $0.2543(7)$ & $0.4286(6)$ & $12.4(5)$ & C55 & $0.5360(8)$ & $-0.2334(8)$ & $0.0125(9)$ & $7.8(5)^{*}$ \\
\hline $\mathrm{O} 92$ & $0.7066(6)$ & $0.2838(8)$ & $0.5313(8)$ & $15.9(6)$ & C56 & $0.4750(9)$ & $-0.2202(8)$ & $0.0047(9)$ & $7.7(5)^{*}$ \\
\hline 093 & 0.763 (1) & $0.3104(9)$ & 0.482 (1) & $25(1)$ & C84 & $0.7742(8)$ & $0.0180(9)$ & $0.4993(9)$ & $13.9(6)$ \\
\hline 0101 & $0.5545(6)$ & $0.2326(9)$ & $0.7136(7)$ & $15.1(7)$ & $\mathrm{H} 2$ & 0.547 & -0.059 & -0.037 & $6.0^{*}$ \\
\hline 0102 & $0.4474(9)$ & $0.2065(9)$ & 0.669 (1) & $21.5(9)$ & $\mathrm{H} 8$ & 0.434 & 0.132 & -0.141 & $6.0^{*}$ \\
\hline 0103 & 0.469 (1) & 0.278 (1) & $0.688(1)$ & $27(1)$ & $\mathrm{H} 9$ & 0.536 & 0.104 & -0.109 & $6.0^{*}$ \\
\hline $\mathrm{Nl}$ & $0.4705(5)$ & $-0.0918(5)$ & $-0.0635(5)$ & $4.1(3)^{*}$ & $\mathrm{H} 10$ & 0.567 & 0.024 & -0.068 & $6.0^{*}$ \\
\hline NE3 & $0.3562(5)$ & $0.0594(6)$ & 0.6905 & $5.6(4)$ & $\mathrm{H} 12 \mathrm{~A}$ & 0.363 & 0.150 & -0.122 & $6.0^{*}$ \\
\hline NB3 & $0.6680(6)$ & $0.3513(5)$ & $0.6561(6)$ & $6.1(4)$ & $\mathrm{H} 12 \mathrm{~B}$ & 0.308 & 0.133 & -0.112 & $6.0^{*}$ \\
\hline NA3 & $0.6657(8)$ & $0.1641(9)$ & $0.2883(8)$ & $12.9(7)$ & $\mathrm{H} 14$ & 0.341 & 0.194 & -0.227 & $6.0^{*}$ \\
\hline N50 & $0.3958(5)$ & $-0.1520(4)$ & $-0.0401(5)$ & $3.9(2)^{*}$ & $\mathrm{H} 16 \mathrm{~A}$ & 0.320 & 0.246 & -0.317 & $6.0^{*}$ \\
\hline CE2 & $0.3087(7)$ & $0.0528(6)$ & $0.7012(7)$ & $5.7(5)$ & $\mathrm{H} 16 \mathrm{~B}$ & 0.271 & 0.224 & -0.387 & $6.0^{*}$ \\
\hline $\mathrm{C} 2$ & $0.5024(7)$ & $-0.0543(6)$ & $-0.0600(7)$ & $5.0(4)^{*}$ & $\mathrm{H} 16 \mathrm{C}$ & 0.253 & 0.268 & -0.354 & $6.0^{*}$ \\
\hline $\mathrm{CA} 2$ & $0.3478(9)$ & 0.136 & $0.265(1)$ & $23(1)$ & $\mathrm{H} 17$ & 0.166 & 0.192 & -0.384 & $6.0^{*}$ \\
\hline $\mathrm{CB} 2$ & 0.7009 (7) & $0.3810(6)$ & $0.7091(7)$ & $4.9(4)$ & $\mathrm{H} 21 \mathrm{~A}$ & 0.164 & 0.104 & -0.142 & $6.0^{*}$ \\
\hline $\mathrm{C} 3$ & $0.4807(6)$ & $-0.0025(6)$ & $-0.0865(6)$ & $4.3(3)^{*}$ & H $21 B$ & 0.204 & 0.152 & -0.136 & $6.0^{*}$ \\
\hline CB4 & $0.6357(7)$ & $0.3717(6)$ & $0.5857(7)$ & $5.2(5)$ & $\mathrm{H} 21 \mathrm{C}$ & 0.138 & 0.145 & -0.199 & $6.0^{*}$ \\
\hline CA4 & $0.666(2)$ & $0.219(1)$ & 0.285 (1) & $16(1)$ & $\mathrm{H} 23$ & 0.064 & 0.194 & -0.353 & $6.0^{*}$ \\
\hline CE4 & $0.4079(7)$ & $0.0235(8)$ & $0.7125(8)$ & $7.4(6)$ & $\mathrm{H} 25 \mathrm{~A}$ & -0.047 & 0.205 & -0.395 & $6.0^{*}$ \\
\hline CE5 & $0.3560(9)$ & $0.1064(8)$ & $0.6531(9)$ & $9.6(6)$ & $\mathrm{H} 25 \mathrm{~B}$ & -0.090 & 0.164 & -0.445 & $6.0^{*}$ \\
\hline $\mathrm{C} 5$ & $0.4168(5)$ & $0.0131(5)$ & $-0.1110(6)$ & $3.2(3)^{*}$ & $\mathrm{H} 25 \mathrm{C}$ & -0.076 & 0.164 & -0.370 & $6.0^{*}$ \\
\hline CA5 & 0.678 (1) & $0.140(1)$ & $0.356(1)$ & $13(1)$ & $\mathrm{H} 26$ & -0.049 & 0.071 & -0.397 & $6.0^{*}$ \\
\hline CB5 & $0.660(1)$ & $0.2960(7)$ & $0.665(1)$ & $11.0(9)$ & $\mathrm{H} 30 \mathrm{~A}$ & 0.191 & 0.004 & -0.374 & $6.0^{*}$ \\
\hline $\mathrm{C} 7$ & $0.4029(6)$ & $0.0637(5)$ & $-0.1273(6)$ & $3.6(3)^{*}$ & $\mathrm{H} 30 \mathrm{~B}$ & 0.118 & 0.010 & -0.415 & $6.0^{*}$ \\
\hline $\mathrm{C} 8$ & $0.4458(6)$ & $0.0972(6)$ & $-0.1274(7)$ & $5.0(4)^{*}$ & $\mathrm{H} 30 \mathrm{C}$ & 0.160 & 0.056 & -0.407 & $6.0^{*}$ \\
\hline $\mathrm{C} 9$ & $0.5067(7)$ & $0.0814(6)$ & $-0.1067(7)$ & $5.5(4)^{*}$ & $\mathrm{H} 32$ & -0.023 & -0.017 & -0.454 & $6.0^{*}$ \\
\hline $\mathrm{C} 10$ & $0.5241(7)$ & $0.0341(6)$ & $-0.0850(7)$ & $4.8(4)^{*}$ & $\mathrm{H} 34 \mathrm{~A}$ & -0.051 & -0.151 & -0.456 & $6.0^{*}$ \\
\hline $\mathrm{Cl} 2$ & $0.3261(6)$ & $0.1304(5)$ & $-0.1404(7)$ & $4.4(3)^{*}$ & $\mathrm{H} 34 \mathrm{~B}$ & -0.092 & -0.103 & -0.490 & $6.0^{*}$ \\
\hline $\mathrm{C} 13$ & $0.2808(5)$ & $0.1494(5)$ & $-0.2092(6)$ & $3.2(3)^{*}$ & $\mathrm{H} 34 \mathrm{C}$ & -0.034 & -0.114 & -0.500 & $6.0^{*}$ \\
\hline $\mathrm{C} 14$ & $0.2987(6)$ & $0.1832(5)$ & $-0.2467(6)$ & $3.9(3)^{*}$ & H35 & 0.024 & -0.143 & -0.338 & $6.0^{*}$ \\
\hline $\mathrm{Cl} 5$ & $0.2566(6)$ & $0.2001(6)$ & $-0.3098(7)$ & $4.1(3)^{*}$ & $\mathrm{H} 39 \mathrm{~A}$ & 0.117 & 0.040 & -0.159 & $6.0^{*}$ \\
\hline $\mathrm{C} 16$ & $0.2768(6)$ & $0.2381(6)$ & $-0.3448(7)$ & $5.3(4)^{*}$ & H39B & 0.052 & 0.043 & -0.223 & $6.0^{*}$ \\
\hline $\mathrm{C} 17$ & $0.1953(6)$ & $0.1817(5)$ & $-0.3389(7)$ & $4.1(3)^{*}$ & $\mathrm{H} 39 \mathrm{C}$ & 0.073 & -0.006 & -0.179 & $6.0^{*}$ \\
\hline $\mathrm{C} 18$ & $0.1753(5)$ & $0.1481(5)$ & $-0.3051(6)$ & $3.0(3)^{*}$ & $\mathrm{H} 40 \mathrm{~A}$ & 0.088 & -0.141 & -0.221 & $6.0^{*}$ \\
\hline $\mathrm{C} 19$ & $0.2215(6)$ & $0.1333(5)$ & $-0.2389(6)$ & $3.3(3)^{*}$ & $\mathrm{H} 40 \mathrm{~B}$ & 0.079 & -0.091 & -0.189 & $6.0^{*}$ \\
\hline $\mathrm{C} 21$ & $0.1748(6)$ & $0.1279(6)$ & $-0.1669(7)$ & $5.6(4)^{*}$ & $\mathrm{H} 45$ & 0.123 & -0.148 & -0.107 & $6.0^{*}$ \\
\hline $\mathrm{C} 22$ & $0.1115(5)$ & $0.1261(5)$ & $-0.3350(6)$ & $3.3(3)^{*}$ & $\mathrm{H} 46$ & 0.188 & -0.195 & -0.006 & $6.0^{*}$ \\
\hline $\mathrm{C} 23$ & $0.0592(6)$ & $0.1583(6)$ & $-0.3549(6)$ & $4.2(3)^{*}$ & $\mathrm{H} 47$ & 0.293 & -0.197 & 0.034 & $6.0^{*}$ \\
\hline C24 & $-0.0003(6)$ & $0.1356(6)$ & $-0.3792(7)$ & $4.3(3)^{*}$ & H49 & 0.384 & -0.188 & 0.021 & $6.0^{*}$ \\
\hline $\mathrm{C} 25$ & $-0.0594(6)$ & $0.1706(6)$ & $-0.3993(7)$ & $5.3(4)^{*}$ & H53 & 0.581 & -0.134 & -0.032 & $6.0^{*}$ \\
\hline $\mathrm{C} 26$ & $-0.0079(6)$ & $0.0849(5)$ & $-0.3807(6)$ & $4.1(3)^{*}$ & H54 & 0.608 & -0.220 & 0.005 & $6.0^{*}$ \\
\hline $\mathrm{C} 27$ & $0.0446(6)$ & $0.0520(5)$ & $-0.3596(6)$ & $3.5(3)^{*}$ & H55 & 0.552 & -0.266 & 0.030 & $6.0^{*}$ \\
\hline $\mathrm{C} 28$ & $0.1030(5)$ & $0.0748(5)$ & $-0.3394(6)$ & $2.8(3)^{*}$ & $\mathrm{H} 56$ & 0.449 & -0.240 & 0.016 & $6.0^{*}$ \\
\hline
\end{tabular}

${ }^{a}$ See footnote $a$ of Table III.

therefore the reduction will be easier. Our results indicate that the association constants of the $\mathrm{Li}^{+}$complexes are smaller than those of the complexes of other hard cations.

Electron Spin Resonance. Electron spin resonance spectra of the copper-containing complexes $12,16,16 \cdot \mathrm{K}^{+} \mathrm{Pic}^{-40} \mathbf{2 4}$, and 27 were obtained at room temperature and a frequency of approx-

(40) Anal. Calcd for 16. $\mathrm{K}^{+} \mathrm{Pic}^{-}\left(\mathrm{C}_{30} \mathrm{H}_{30} \mathrm{CuKN}_{5} \mathrm{O}_{13} \cdot 0.5 \mathrm{H}_{2} \mathrm{O}\right): \mathrm{C}, 46.19 ; \mathrm{H}$, 3.85; N, 8.90. Found: $\mathrm{C}, 46.18 ; \mathrm{H}, 4.00 ; \mathrm{N}, 8.98$. imately $35 \mathrm{GHz}$. $\mathrm{Cu}^{2+}$ has the electronic configuration $3 \mathrm{~d}^{9}$ with ground state $\mathrm{t}_{2}{ }^{6} \mathrm{e}^{3}$, and this situation may be regarded as a single hole in an e orbital. 41 The ground state in the octahedral field is an orbital doublet, which is split by a tetragonal field into two Kramers doublets. In the case of an elongated octahedron $\mathbf{d}_{x^{2}-y^{2}}$ is the ground-state doublet with approximate $g$ factors $g_{\|}=2-$

(41) Orton, J. W. An Introduction to Transition Group Ions in Crystals; Iliffe Books: London, 1968. 
Table VII. Polarographic Data for Reduction at a Dropping Mercury Electrode at $20{ }^{\circ} \mathrm{C}$ in $0.1 \mathrm{M} \mathrm{Et}_{4} \mathrm{~N}^{+} \mathrm{ClO}_{4}{ }^{-}$in $\mathrm{DMSO}$ vs $\mathrm{Ag} / \mathrm{AgCl}$

\begin{tabular}{|c|c|c|c|c|c|c|c|c|}
\hline \multirow[b]{2}{*}{ compd } & \multirow{2}{*}{$\begin{array}{l}\text { cation added }{ }^{a} \\
\text { (amt, equiv) }\end{array}$} & \multicolumn{2}{|c|}{$E_{1 / 2}, \mathrm{~V}$} & \multicolumn{2}{|c|}{$i_{\mathrm{d}}, \mu \mathrm{A}$} & \multicolumn{2}{|c|}{ slope of log plot, $\mathrm{mV}$} & \multirow[b]{2}{*}{ concn, $\mathrm{mM}$} \\
\hline & & Ist wave & 2nd wave & 1st wave & 2nd wave & Ist wave & 2nd wave & \\
\hline $14(\mathrm{Ni})$ & & -1.469 & -1.669 & 0.718 & 1.543 & 60 & 47 & 2.00 \\
\hline $10(\mathrm{Ni}, \mathrm{Ba})$ & & -1.338 & & 1.396 & & 59 & & 2.10 \\
\hline $14(\mathrm{Ni})$ & $\mathrm{K}^{+}(1)$ & -1.368 & -1.504 & 0.460 & 2.063 & 53 & 96 & 1.82 \\
\hline $14(\mathrm{Ni})$ & $\mathrm{K}^{+}(2)$ & -1.367 & -1.503 & 0.448 & 1.990 & 55 & 99 & 1.67 \\
\hline $14(\mathrm{Ni})$ & $\mathrm{Na}^{+}(1)$ & -1.366 & -1.538 & 0.746 & 2.300 & 54 & 140 & 1.82 \\
\hline $14(\mathrm{Ni})$ & $\mathrm{Na}^{+}(2)$ & -1.401 & -1.530 & 0.651 & 2.184 & 50 & 130 & 1.67 \\
\hline $14(\mathrm{Ni})$ & $\mathrm{Li}^{+}(1)$ & -1.465 & -1.654 & 0.755 & 1.310 & 53 & 92 & 1.82 \\
\hline $14(\mathrm{Ni})$ & $\mathrm{Li}^{+}(2)$ & -1.459 & -1.631 & 0.710 & 1.554 & 59 & 114 & 1.67 \\
\hline $14(\mathrm{Ni})$ & $\mathrm{Li}^{+}(3)$ & -1.459 & -1.633 & 0.658 & 1.843 & 57 & 114 & 1.54 \\
\hline $14(\mathrm{Ni})$ & $\mathrm{Li}^{+}(4)$ & -1.457 & -1.610 & 0.645 & 1.477 & 61 & 119 & 1.43 \\
\hline $14(\mathrm{Ni})$ & $\mathrm{Li}^{+}(5)$ & -1.456 & -1.615 & 0.572 & 1.620 & 58 & 133 & 1.33 \\
\hline $\begin{array}{l}15(\mathrm{Ni}) \\
11(\mathrm{Ni}, \mathrm{Ba})\end{array}$ & & $\begin{array}{l}-1.451 \\
-1.359^{b}\end{array}$ & -1.621 & $\begin{array}{l}0.462 \\
2.168\end{array}$ & 0.976 & $\begin{array}{r}76 \\
104\end{array}$ & 65 & $\begin{array}{l}2.00 \\
2.52\end{array}$ \\
\hline $15(\mathrm{Ni})$ & $\mathrm{K}^{+}(1)$ & -1.394 & -1.510 & 0.463 & 1.565 & 65 & 114 & 1.82 \\
\hline $15(\mathrm{Ni})$ & $\mathrm{K}^{+}(2)$ & -1.394 & -1.515 & 0.410 & 1.485 & 65 & 116 & 1.67 \\
\hline $15(\mathrm{Ni})$ & $\mathrm{Na}^{+}(1)$ & -1.422 & -1.536 & 0.681 & 1.776 & 69 & 131 & 1.82 \\
\hline $15(\mathrm{Ni})$ & $\mathrm{Na}^{+}(2)$ & -1.413 & -1.530 & 0.569 & 1.647 & 68 & 128 & 1.67 \\
\hline $15(\mathrm{Ni})$ & $\mathrm{Na}^{+}(3)$ & -1.409 & -1.528 & 0.470 & 1.464 & 61 & 128 & 1.54 \\
\hline $15(\mathrm{Ni})$ & $\mathrm{Li}^{+}(1)$ & -1.450 & -1.602 & 0.570 & 1.064 & 72 & 99 & 1.82 \\
\hline $15(\mathrm{Ni})$ & $\mathrm{Li}^{+}(2)$ & -1.451 & -1.597 & 0.537 & 1.180 & 75 & 113 & 1.67 \\
\hline $15(\mathrm{Ni})$ & $\mathrm{Li}^{+}(3)$ & -1.451 & -1.585 & 0.509 & 1.080 & 76 & 111 & 1.54 \\
\hline $15(\mathrm{Ni})$ & $\mathrm{Li}^{+}(4)$ & -1.455 & -1.581 & 0.481 & 1.097 & 82 & 117 & 1.43 \\
\hline $15(\mathrm{Ni})$ & $\mathrm{Li}^{+}(5)$ & -1.446 & -1.579 & 0.410 & 1.060 & 74 & 118 & 1.33 \\
\hline $16(\mathrm{Cu})$ & & -1.332 & & 1.935 & & 68 & & 2.00 \\
\hline $12(\mathrm{Cu}, \mathrm{Ba})$ & & -1.119 & & 1.391 & & 51 & & 2.30 \\
\hline $16(\mathrm{Cu})$ & $\mathrm{K}^{+}(1)$ & -1.278 & & 1.721 & & 58 & & 1.82 \\
\hline $16(\mathrm{Cu})$ & $\mathrm{K}^{+}(2)$ & -1.277 & & 1.563 & & 58 & & 1.67 \\
\hline $16(\mathrm{Cu})$ & $\mathrm{Na}^{+}(1)$ & -1.280 & & 1.674 & & 59 & & 1.82 \\
\hline $16(\mathrm{Cu})$ & $\mathrm{Na}^{+}(2)$ & -1.279 & & 1.510 & & 59 & & 1.67 \\
\hline $16(\mathrm{Cu})$ & $\mathrm{Li}^{+}(1)$ & -1.319 & & 1.902 & & 74 & & 1.82 \\
\hline $16(\mathrm{Cu})$ & $\mathrm{Li}^{+}(2)$ & -1.304 & & 1.694 & & 75 & & 1.67 \\
\hline $16(\mathrm{Cu})$ & $\mathrm{Li}^{+}(3)$ & -1.295 & & 1.577 & & 77 & & 1.54 \\
\hline $16(\mathrm{Cu})$ & $\mathrm{Li}^{+}(4)$ & -1.291 & & 1.509 & & 82 & & 1.43 \\
\hline $16(\mathrm{Cu})$ & $\mathrm{Li}^{+}(5)$ & -1.286 & & 1.409 & & 81 & & 1.33 \\
\hline $17(\mathrm{Cu})$ & & -1.265 & & 1.922 & & 65 & & 2.00 \\
\hline $13(\mathrm{Cu}, \mathrm{Ba})$ & & -1.108 & & 2.526 & & 58 & & 3.64 \\
\hline $17(\mathrm{Cu})$ & $\mathrm{K}^{+}(1)$ & -1.257 & & 1.725 & & 64 & & 1.82 \\
\hline $17(\mathrm{Cu})$ & $\mathrm{K}^{+}(2)$ & -1.256 & & 1.571 & & 63 & & 1.67 \\
\hline $17(\mathrm{Cu})$ & $\mathrm{Na}^{+}(1)$ & -1.251 & & 1.753 & & 66 & & 1.82 \\
\hline $17(\mathrm{Cu})$ & $\mathrm{Na}^{+}(2)$ & -1.246 & & 1.610 & & 65 & & 1.67 \\
\hline $17(\mathrm{Cu})$ & $\mathrm{Na}^{+}(3)$ & -1.243 & & 1.470 & & 64 & & 1.54 \\
\hline $17(\mathrm{Cu})$ & $\mathrm{Na}^{+}(4)$ & -1.240 & & 1.371 & & 64 & & 1.43 \\
\hline $17(\mathrm{Cu})$ & $\mathrm{Li}^{+}(1)$ & -1.253 & & 1.875 & & 74 & & 1.82 \\
\hline $17(\mathrm{Cu})$ & $\mathrm{Li}^{+}(2)$ & -1.241 & & 1.687 & & 74 & & 1.67 \\
\hline $17(\mathrm{Cu})$ & $\mathrm{Li}^{+}(3)$ & -1.237 & & 1.642 & & 81 & & 1.54 \\
\hline $17(\mathrm{Cu})$ & $\mathrm{Li}^{+}(4)$ & -1.231 & & 1.503 & & 80 & & 1.43 \\
\hline $17(\mathrm{Cu})$ & $\mathrm{Li}^{+}(5)$ & -1.224 & & 1.395 & & 78 & & 1.33 \\
\hline $26(\mathrm{Ni})$ & & $-1.082^{c}$ & $-1.247^{c}$ & $1.174^{c}$ & $0.700^{c}$ & $80^{\circ}$ & $55^{c}$ & 2.00 \\
\hline $23(\mathrm{Ni}, \mathrm{Ba})$ & & -1.018 & & 0.629 & & 55 & & 0.96 \\
\hline $26(\mathrm{Ni})$ & $\mathrm{K}^{+}(1)$ & -1.191 & & 0.832 & & 57 & & 1.82 \\
\hline $26(\mathrm{Ni})$ & $\mathrm{K}^{+}(2)$ & -1.191 & & 0.774 & & 56 & & 1.67 \\
\hline $27(\mathrm{Cu})$ & & -1.008 & & 0.727 & & 54 & & 2.00 \\
\hline $24(\mathrm{Cu}, \mathrm{Ba})$ & & -0.821 & & 0.662 & & 53 & & 0.97 \\
\hline $27(\mathrm{Cu})$ & $\mathrm{K}^{+}(1)$ & -1.008 & & 0.624 & & 51 & & 1.82 \\
\hline $27(\mathrm{Cu})$ & $\mathrm{K}^{+}(2)$ & -1.008 & & 0.600 & & 53 & & 1.67 \\
\hline $25(\mathrm{Zn}, \mathrm{Ba})$ & & -1.466 & & 0.959 & & 42 & & 1.44 \\
\hline $29(\mathrm{Ni})$ & & $-1.448^{c}$ & $-1.552^{c}$ & $0.750^{c}$ & $0.750^{c}$ & $84^{c}$ & $40^{c}$ & 2.00 \\
\hline $28(\mathrm{Ni}, \mathrm{Ba})$ & & -1.237 & & 1.046 & & 68 & & 1.34 \\
\hline $29(\mathrm{Ni})$ & $\mathrm{K}^{+}(\mathrm{l})$ & -1.432 & & 1.291 & & 59 & & 1.82 \\
\hline $29(\mathrm{Ni})$ & $\mathrm{K}^{+}(2)$ & -1.432 & & 1.171 & & & & 1.67 \\
\hline
\end{tabular}

${ }^{a}$ The alkali-metal cations were added as their perchlorate salts dissolved in $0.1 \mathrm{M} \mathrm{Et}_{4}{ }^{+} \mathrm{ClO}_{4}^{-}$in DMSO. ${ }^{b} \mathrm{~A}$ prewave seems to be present. 'Determined manually, because the evaluating program was not able to handle the two waves that are present.

$8 \lambda / \Delta$ and $g_{\perp}=2-2 \lambda / \Delta$, where $\lambda$ ( $<0$ for copper) is the spinorbit coupling constant and $\Delta$ the cubic field splitting. Although the symmetry around the $\mathrm{Cu}^{2+}$ site is much lower, the ESR spectra resemble those of an axially distorted octahedron. This can be seen in Figure 7 , in which the measured spectrum of the copper complex $\mathbf{2 7}$ is presented.

In order to check whether our spectrum can indeed be described by an axial spin Hamiltonian, we performed several simulations where the $g$ factors $g_{\|}$and $g_{\perp}$, the hyperfine field splittings $A_{\|}$ and $A_{\perp}$, and the line widths $\Gamma_{\|}$and $\Gamma_{\perp}$ were varied. Furthermore, both Lorentzian and Gaussian line profile functions were used. A typical simulation for the copper complex 27 is also presented in Figure 7. The values for the parameters used in this particular simulation are $g_{\|}=2.213, g_{\perp}=2.0568, A_{\|}=560 \mathrm{MHz}, A_{\perp}=$ 0 , and $\Gamma_{\|}=\Gamma_{\perp}=340 \mathrm{MHz}$, with a Lorentzian line profile (the measured frequency $\nu=34.841 \mathrm{GHz}$ ). The fit was optimized 
Table VIII. Shifts ( $\mathrm{mV}$ ) in the Half-Wave Potential Quoted Relative to the Monocopper Complexes 16, 17, and 27 upon Complexation with Cations

\begin{tabular}{lrrr}
\hline & \multicolumn{1}{c}{16} & \multicolumn{1}{c}{17} & \multicolumn{1}{c}{$\mathbf{2 7}$} \\
\hline $\mathrm{Ba}^{2+}$ & $213(1)^{a}$ & $157(1)$ & $187(1)$ \\
$\mathrm{K}^{+}$ & $54(1)$ & $9(1)$ & $0(2)$ \\
$\mathrm{Na}^{+}$ & $52(1)$ & $25(4)$ & $b$ \\
$\mathrm{Li}^{+}$ & $46(5)$ & $41(5)$ & $b$
\end{tabular}

${ }^{a}$ In parentheses is given the number of equivalents of cations present in solution. ${ }^{b}$ Not determined.

Table IX. Shift of $g_{\perp}$ with Respect to $g_{e}$

\begin{tabular}{lccc}
\hline complex & $g_{\perp}-g_{e}$ & complex & $g_{\perp}-g_{e}$ \\
\hline 16 & +0.0527 & 27 & +0.0545 \\
12 & +0.0426 & 24 & +0.0494 \\
$16 \cdot \mathrm{K}^{+}$Pic $^{-a}$ & +0.0437 & &
\end{tabular}

${ }^{a} \mathrm{~K}^{+} \mathrm{Pic}^{-}=$potassium picrate.

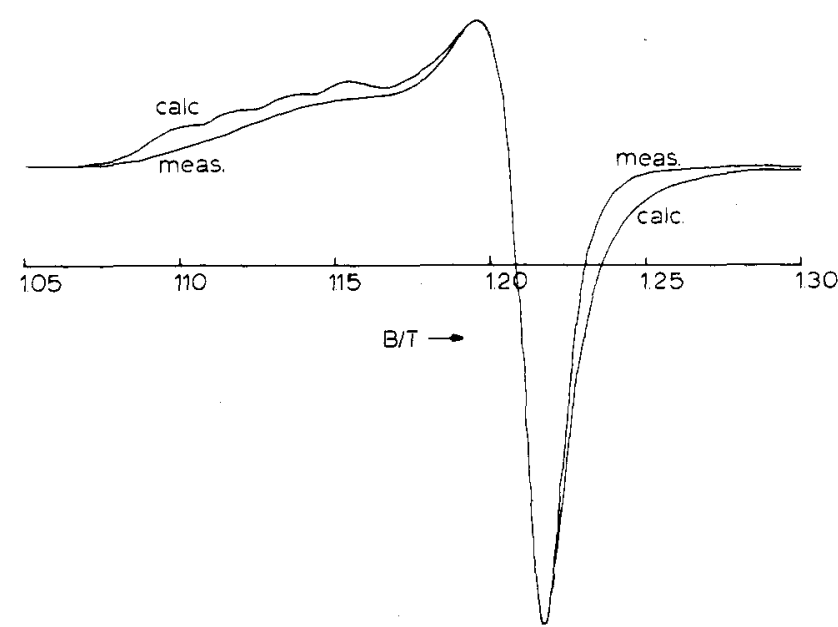

Figure 7. Measured and calculated ESR spectra of the copper complex 27 at room temperature. For the parameters of the calculated spectrum see text.

to give the correct value for $B_{\perp}$, the peak-to-peak line width, and the ratio of the positive-to-negative amplitude around $B_{\perp}$. Due to hardly resolved hyperfine field splitting around $B_{\|}$both $g_{\|}$and $A_{\|}$are difficult to obtain from simulations. Nevertheless it can be seen that the simulation spectrum is a reasonable fit to the measured spectrum. The value we find for $g_{\perp}$ is very insensitive to changes in the other parameters, and therefore only this parameter was used to obtain a $g$ shift with respect to the freeelectron value. The shifts $\Delta g$ of $g_{\perp}$ with respect to the free-electron value $\left(g_{\mathrm{e}}\right)$ of the five copper complexes $12,16,16 \cdot \mathrm{K}^{+} \mathrm{Pic}^{-}, 24$, and 27 are given in Table IX. The error in $\Delta g$ is estimated to be \pm 0.003 and is obtained by repeated calibrations against a known standard (the Varian pitch with $g=2.0028$ ). From Table IX it is obvious that the $g$ shifts decrease upon complexation with either $\mathrm{Ba}^{2+}$ or $\mathrm{K}^{+}$. This shift more or less parallels the difference in $E_{1 / 2}$; i.e., the shift induced by $\mathrm{Ba}^{2+}$ is larger than that by $\mathrm{K}^{+}$. Furthermore the difference between 16 and 12 is somewhat larger than the difference between 27 and 24 just as for the $E_{1 / 2}$ values.

\section{Conclusions}

The $\mathrm{Ba}^{2+}$-templated $(1: 1)$ macrocyclization of the dialdehydes $\mathbf{7}, \mathbf{8}$, and 20 with the diamines 9 and 21 offers a convenient route to heterodinuclear ligands with two very different cavities. The resulting barium complexes can be simply transformed to heterodinuclear complexes upon reaction with nickel, copper, or zinc acetate. The coordination of the zinc cation is square pyramidal in 25.3DMF whereas the nickel coordination is square planar in the complexes $3 \cdot \mathrm{MeOH}, 10 \cdot \mathrm{H}_{2} \mathrm{O}$, and $14 \cdot \mathrm{Na}^{+} \mathrm{Pic}^{-}$. Our electrochemical studies show that the redox properties of the complexed transition-metal cation are strongly affected by the complexation of a hard cation in the polyether cavity. Cyclic voltammetry showed that the one-electron reduction of the nickel/barium complex 10, the copper/barium complex 12, and the nickel complex 15 is chemically reversible but electrochemically irreversible at the applied scan rates. The two-electron reduction of the zinc/barium complex 25 is both electrochemically and chemically irreversible. The ESR measurements also showed the influence of complexed hard cations on the properties of the complexed copper cation. We are currently studying the interaction of these heterodinuclear complexes with organic guests and small molecules like $\mathrm{CO}_{2}, \mathrm{CO}, \mathrm{NO}_{x}$, and $\mathrm{SO}_{x}$. Our ultimate goal is the application of a heterodinuclear complex as a bifunctional catalyst.

\section{Experimental Section}

Melting points were determined with a Reichert melting point apparatus and are uncorrected. ${ }^{1} \mathrm{H}$ NMR spectra ${ }^{42}$ were recorded with a Bruker WP-80 spectrometer, and ${ }^{13} \mathrm{C}$ NMR spectra ${ }^{42}$ were recorded with a Nicolet NT-200 WB spectrometer. The spectra were recorded in $\mathrm{CDCl}_{3}$ with $\mathrm{Me}_{4} \mathrm{Si}$ as an internal standard unless otherwise stated. Mass spectra were obtained with a Varian MAT $311 \mathrm{~A}$ spectrometer. Fast atom bombardment mass spectra of the compounds 23-27 were obtained with a VG Micromass ZAB-2HF mass spectrometer. The samples were loaded in a thioglycerol solution. Infrared spectra were recorded with a Perkin-Elmer 257 (compound 20) or a Nicolet 5SXC FT-IR spectrophotometer (compounds 1-3, 10-13, 22, 23-29). Electron spin resonance spectra were obtained with a Varian E-112Q spectrometer and were recorded at room temperature. Elemental analyses were carried out by the Department of Chemical Analysis of our institute.

All chemicals were reagent grade and were used without further purification. DIP refers to diisopropyl ether, petroleum ether 40-60 to petroleum ether with boiling fraction $40-60^{\circ} \mathrm{C}$, and petroleum ether $60-80$ to petroleum ether with boiling fraction $60-80^{\circ} \mathrm{C}$. DMSO refers to dimethyl sulfoxide and THF to tetrahydrofuran. Dropwise addition over a period of several hours was always carried out with a perfusor.

$(\mu-(9,10,12,13,15,16,18,19,21,22$-Decahydro-3,7:24,28-dimetheno$8,11,14,17,20,23,1,30$-benzohexaoxadiazacyclodotriacontine-35,36-diolato $\left.(2-) \cdot N^{1}, N^{30}, O^{35}, O^{36}: O^{8}, O^{11}, O^{14}, O^{17}, O^{20}, O^{23}, O^{35}, O^{36}\right)$ )(nickel)barium(2+) Diperchlorate (1). Complex $4(0.45 \mathrm{~g}, 0.50 \mathrm{mmol})$ was dissolved in $50 \mathrm{~mL}$ of $\mathrm{MeOH}$, and a solution of $\mathrm{Ni}(\mathrm{OAc})_{2} \cdot 4 \mathrm{H}_{2} \mathrm{O}(0.12 \mathrm{~g}, 0.50$ $\mathrm{mmol}$ ) in $50 \mathrm{~mL}$ of $\mathrm{MeOH}$ was added at room temperature. The mixture turned deep red and was heated to reflux for $5 \mathrm{~min}$, filtered while hot, and concentrated to $50 \mathrm{~mL}$. After addition of $50 \mathrm{~mL}$ of petroleum ether 40-60 the red product crystallized and was filtered off: yield $56 \%$; $\mathrm{mp}$ $>300^{\circ} \mathrm{C} ;{ }^{1} \mathrm{H}$ NMR (DMSO- $d_{6} ; 358 \mathrm{~K}$ ) $\delta 9.6$ (bs, $2 \mathrm{H}, \mathrm{N}=\mathrm{CH}$ ), 8.2-8.0 (m, $\left.2 \mathrm{H}, \mathrm{Ar}^{\prime} \mathrm{H}\right), 7.5-7.3\left(\mathrm{~m}, 2 \mathrm{H}, \mathrm{Ar}^{\prime} \mathrm{H}\right), 7.5-7.0(\mathrm{~m}, 4 \mathrm{H}, \mathrm{Ar} \mathrm{H})$, 6.8-6.5 (m, $2 \mathrm{H}$, Ar H) $4.3-3.8\left(\mathrm{~m}, 8 \mathrm{H}, \mathrm{OCH}_{2}\right), 3.7-3.5(\mathrm{~m}, 12 \mathrm{H}$, $\left.\mathrm{OCH}_{2}\right) ;{ }^{13} \mathrm{C}$ NMR (DMSO-d $\left.6358 \mathrm{~K}\right) \delta 157.3(\mathrm{~d}, \mathrm{~N}=\mathrm{CH}), 154.9(\mathrm{~s}$, Ar C-2), 151.8 (s, Ar C-3), 140.2 (s, Ar C-1,2), 127.9, 126.3 (d, Ar C-6, $\mathrm{Ar}^{\prime} \mathrm{C}-4,5$ ), 120.6 (s, $\mathrm{Ar} \mathrm{C}-1$ ), 117.5, 116.8, 115.3 (d, Ar C-4,5, $\mathrm{Ar}^{\prime}$ C-3,6), 68.7-67.1 (t, $\left.\mathrm{OCH}_{2}\right)$; IR $(\mathrm{KBr}) 1613(\mathrm{~N}=\mathrm{C}) \mathrm{cm}^{-1}$; mass spectrum $m / e$ 606.141 $\left(\mathrm{M}^{+}-\mathrm{Ba}\left(\mathrm{ClO}_{4}\right)_{2}\right.$, calcd 606.151). Anal. Calcd for $\mathrm{C}_{30} \mathrm{H}_{32} \mathrm{BaCl}_{2} \mathrm{~N}_{2} \mathrm{NiO}_{16}: \mathrm{C}, 38.19 ; \mathrm{H}, 3.42 ; \mathrm{N}, 2.97$. Found: C, 38.36; $\mathrm{H}, 3.53 ; \mathrm{N}, 3.21$.

$(\mu-(9,10,12,13,15,16,18,19,21,22,24,25-D o d e c a h y d r 0-3,7: 27,31$-dimetheno-8,11,14,17,20,23,26,1,33-benzoheptaoxadia zacyclopentatriacontine-38,39-diolato $(2-)-N^{1}, N^{33}, O^{38}, O^{39}: O^{8}, O^{11}, O^{14}, O^{17}, O^{20}, O^{23}$ $\left.O^{26}, O^{38}, O^{39}\right)$ )(nickel)barium(2+) Diperchlorate (2). Complex $5(0.93$ $\mathrm{g}, 1.00 \mathrm{mmol}$ ) was dissolved in $100 \mathrm{~mL}$ of refluxing $\mathrm{MeOH}$, and a solution of $\mathrm{Ni}(\mathrm{OAC})_{2} \cdot 4 \mathrm{H}_{2} \mathrm{O}(0.25 \mathrm{~g}, 1.00 \mathrm{mmol})$ in $50 \mathrm{~mL}$ of $\mathrm{MeOH}$ was added to the hot solution. The mixture turned deep red and was refluxed for $5 \mathrm{~min}$. When the mixture was cooled, the red product precipitated and was filtered off: yield $72 \%$; mp $>310^{\circ} \mathrm{C}$; ' $\mathrm{H}$ NMR (DMSO- $d_{6}$; $353 \mathrm{~K}) \delta 9.55(\mathrm{~s}, 2 \mathrm{H}, \mathrm{N}=\mathrm{CH}), 8.2-8.0\left(\mathrm{~m}, 2 \mathrm{H}, \mathrm{Ar}^{\prime} \mathrm{H}\right), 7.5-7.3(\mathrm{~m}$ $\left.2 \mathrm{H}, \mathrm{Ar}^{\prime} \mathrm{H}\right), 7.3-6.5(\mathrm{~m}, 6 \mathrm{H}, \mathrm{Ar} \mathrm{H}), 4.3-4.1\left(\mathrm{~m}, 4 \mathrm{H}, \mathrm{ArOCH}_{2}\right)$, 3.9-3.7 (m, $\left.4 \mathrm{H}, \mathrm{OCH}_{2}\right), 3.7-3.4\left(\mathrm{~m}, 16 \mathrm{H}, \mathrm{OCH}_{2}\right) ;{ }^{13} \mathrm{C} \mathrm{NMR}$ $\left(\mathrm{DMSO}-d_{6} ; 353 \mathrm{~K}\right) \delta 156.4(\mathrm{~d}, \mathrm{~N}=\mathrm{CH}), 155.0(\mathrm{~s}, \mathrm{Ar} \mathrm{C}-2), 150.3(\mathrm{~s}, \mathrm{Ar}$ C-3), 140.2 (s, $\left.\mathrm{Ar}^{\prime} \mathrm{C}-1,2\right), 127.2,126.0$ (d, Ar C-6, $\left.\mathrm{Ar}^{\prime} \mathrm{C}-4,5\right), 120.1$ (s, Ar C-1), 117.9, 115.9, 114.7 (d, Ar C-4,5, $\left.\mathrm{Ar}^{\prime} \mathrm{C}-3,6\right), 68.9-67.1$ (t, $\left.\mathrm{OCH}_{2}\right) ; \mathrm{IR}(\mathrm{KBr}) 1614(\mathrm{~N}=\mathrm{C}) \mathrm{cm}^{-1}$; mass spectrum $m / e$ 650.175 $\left(\mathrm{M}^{+}\right.$ - $\mathrm{Ba}\left(\mathrm{ClO}_{4}\right)_{2}$, calcd 650.177). Anal. Calcd for $\mathrm{C}_{32} \mathrm{H}_{36} \mathrm{BaCl}_{2} \mathrm{~N}_{2} \mathrm{NiO}_{17}$. $\mathrm{H}_{2} \mathrm{O}: \mathrm{C}, 38.22 ; \mathrm{H}, 3.81 ; \mathrm{N}, 2.79$. Found: $\mathrm{C}, 38.37 ; \mathrm{H}, 3.76 ; \mathrm{N}, 2.64$.

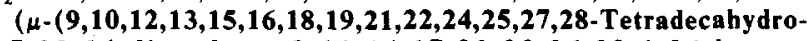
$3,7: 30,34$-dime theno- $8,11,14,17,20,23,26,29,1,36$ - benzooctaoxadiazacyclooctatriacontine-41,42-diolato $(2-)-N^{1}, N^{36}, O^{41}, O^{42}$.

(42) Ar refers to the aromatic ring with a "precursor" aldehyde moiety; $\mathrm{Ar}^{\prime}$ refers to the aromatic ring with two imine bonds or to the outer aromatic rings of the terphenyl; $\mathrm{Ar}^{\prime \prime}$ refers to the inner aromatic ring of the terphenyl. 
$\left.\left.: O^{8}, O^{11}, O^{14}, O^{17}, O^{20}, O^{23}, O^{26}, O^{29}, O^{41}, O^{42}\right)\right)($ nickel) barium (2+) Diperchlorate (3). Complex $6(0.97 \mathrm{~g}, 1.00 \mathrm{mmol})$ was dissolved in $100 \mathrm{~mL}$ of refluxing $\mathrm{MeOH}$, and a solution of $\mathrm{Ni}(\mathrm{OAc})_{2} \cdot 4 \mathrm{H}_{2} \mathrm{O}(0.25 \mathrm{~g}, 1.00$ $\mathrm{mmol}$ ) in $50 \mathrm{~mL}$ of $\mathrm{MeOH}$ was added to the hot solution. The mixture turned deep red and was refluxed for $5 \mathrm{~min}$. When the mixture was cooled and $50 \mathrm{~mL}$ of petroleum ether $40-60$ was added, the red product crystallized and was filtered off: yield $82 \% ; \mathrm{mp}>310^{\circ} \mathrm{C}$; ${ }^{\prime} \mathrm{H}$ NMR (DMSO- $\left.d_{6} ; 353 \mathrm{~K}\right) \delta 9.83(\mathrm{~s}, 2 \mathrm{H}, \mathrm{N}=\mathrm{CH}), 8.2-8.0\left(\mathrm{~m}, 2 \mathrm{H}, \mathrm{Ar}^{\prime} \mathrm{H}\right)$, 7.5-7.3 (m, $\left.2 \mathrm{H}, \mathrm{Ar}^{\prime} \mathrm{H}\right), 7.3-6.6(\mathrm{~m}, 6 \mathrm{H}, \mathrm{Ar} \mathrm{H}), 4.3-4.1(\mathrm{~m}, 4 \mathrm{H}$, $\left.\mathrm{ArOCH}_{2}\right), 4.0-3.8\left(\mathrm{~m}, 4 \mathrm{H}, \mathrm{OCH}_{2}\right), 3.8-3.5\left(\mathrm{~m}, 20 \mathrm{H}, \mathrm{OCH}_{2}\right) ;{ }^{13} \mathrm{C}$ NMR (DMSO- $\left.d_{6} ; 353 \mathrm{~K}\right) \delta 157.0(\mathrm{~d}, \mathrm{~N}=\mathrm{CH}), 153.3(\mathrm{~s}, \mathrm{Ar} \mathrm{C}-2), 149.8$ (s, Ar C-3), 140.4 (s, Ar $^{\prime}$ C-1,2), 127.5, 126.0 (d, Ar C-6, Ar $^{\prime}$ C-4,5), 119.8 (s, Ar C-1), 116,9, 116.0, 115.3 (d, Ar C-4,5, Ar' C-3,6), 69.0-66.5 (t, $\left.\mathrm{OCH}_{2}\right)$; IR $(\mathrm{KBr}) 1612(\mathrm{C}=\mathrm{N}) \mathrm{cm}^{-1}$; mass spectrum $m / e 694.198$ $\left(\mathrm{M}^{+}-\mathrm{Ba}\left(\mathrm{ClO}_{4}\right)_{2}\right.$, calcd 694.204). Anal. Calcd for $\mathrm{C}_{34} \mathrm{H}_{40} \mathrm{BaCl}_{2} \mathrm{~N}_{2} \mathrm{NiO}_{13} \cdot \mathrm{H}_{2} \mathrm{O}: \mathrm{C}, 38.91 ; \mathrm{H}, 4.03 ; \mathrm{N}, 2.67$. Found: $\mathrm{C}$, $39.12 ; \mathrm{H}, 4.06 ; \mathrm{N}, 2.65$.

General Procedure for the Synthesis of Complexes 10-13. To a refluxing solution of $\mathrm{Ba}\left(\mathrm{CF}_{3} \mathrm{SO}_{3}\right)_{2}(0.56 \mathrm{~g}, 1.28 \mathrm{mmol})$ in $100 \mathrm{~mL}$ of $\mathrm{MeOH}$ were added both a solution of the dialdehyde $7(0.50 \mathrm{~g}, 1.28$ mmol) or the dialdehyde $8(0.56 \mathrm{~g}, 1.28 \mathrm{mmol})$ in $25 \mathrm{~mL}$ of THF and a solution of $9(0.11 \mathrm{~g}, 1.28 \mathrm{mmol})$ in $25 \mathrm{~mL}$ of $\mathrm{MeOH}$, dropwise over a period of $3 \mathrm{~h}$. The resulting yellow solution was refluxed for $15 \mathrm{~min}$, after which a solution of 1 equiv of $\mathrm{Ni}(\mathrm{OAc})_{2} \cdot 4 \mathrm{H}_{2} \mathrm{O}$ or $\mathrm{Cu}(\mathrm{OAc})_{2} \cdot \mathrm{H}_{2} \mathrm{O}$ in $\mathrm{MeOH}(10-20 \mathrm{~mL})$ was added in one portion. Subsequently the colored solution was cooled to room temperature and the solvent evaporated. In the cases of $\mathbf{1 0}$ and $\mathbf{1 1}$ the residue was dissolved in EtOH (10 $\mathrm{mL}$ ), and after the addition of some petroleum ether 60-80 the product precipitated. The precipitate was filtered off and was washed once with petroleum ether 60-80 to give the dinuclear complex. In the cases of 12 and 13 the residue was dissolved in a mixture of pyridine $/ \mathrm{CHCl}_{3}(1: 1$; $10 \mathrm{~mL}$ ) and after the addition of some petroleum ether 40-60 the dinuclear complex precipitated, which was filtered off and was washed once with petroleum ether $40-60$.

( $\mu$-(5,5-Dimethyl-13,16,19,22-tetraoxa-3,6-diazatricyclo[21.3.1.1 ${ }^{8,12}$ octacosa- $1(27), 2,6,8,10,12(28), 23,25$-octaene-27,28-diolato(2-)$\left.N^{3}, N^{6}, O^{27}, O^{28}: O^{13}, O^{16}, O^{19}, O^{22}, O^{27}, O^{28}\right)$ )(nickel) barium (2+) bis(trifluoromethanesulfonate) (10): red solid; yield $72 \%$; $\mathrm{mp}>300{ }^{\circ} \mathrm{C}$ $\left(\mathrm{CH}_{3} \mathrm{CN} / \mathrm{DIP}\right){ }^{1} \mathrm{H}$ NMR (DMSO- $\left.d_{6}\right) \delta 7.97,7.91(\mathrm{~s}, 1 \mathrm{H}, \mathrm{N}=\mathrm{CH}$ ), 7.1-7.0 (m, 4 H, Ar H), 6.8-6.6 (m, $2 \mathrm{H}$, Ar H), 4.3-4.0 (m, $4 \mathrm{H}$, ArOCH $), 3.9-3.7\left(\mathrm{~m}, 8 \mathrm{H}, \mathrm{OCH}_{2}\right), 3.39\left(\mathrm{~s}, 2 \mathrm{H}, \mathrm{NCH}_{2}\right), 1.56(\mathrm{~s}, 6 \mathrm{H}$ $\left.\mathrm{CH}_{3}\right)$; IR ( $\left.\mathrm{KBr}\right) 3489(\mathrm{OH}), 1624(\mathrm{~N}=\mathrm{C}) \mathrm{cm}^{-1}$; mass spectrum $\mathrm{m} / \mathrm{e}$ $498.133\left(\mathrm{M}^{+}-\mathrm{Ba}\left(\mathrm{CF}_{3} \mathrm{SO}_{3}\right)_{2}\right.$, calcd 498.130). Anal. Calcd for $\mathrm{C}_{26} \mathrm{H}_{28} \mathrm{BaF}_{6} \mathrm{~N}_{2} \mathrm{NiO}_{12} \mathrm{~S}_{2} \cdot \mathrm{H}_{2} \mathrm{O}: \mathrm{C}, 32.78 ; \mathrm{H}, 3.17 ; \mathrm{N}, 2.94$. Found: $\mathrm{C}$, $32.80 ; \mathrm{H}, 3.04 ; \mathrm{N}, 2.85$

( $\mu$-(5,5-Dimethyl-13,16,19,22,25-pentaoxa-3,6-diazatricyclo[24.3.1.1 $1^{8,12}$ ]hentriacontane-1 $(30), 2,6,8,10,12(31), 26,28$-octaene-30,31diolato $\left.(2-)-N^{3}, N^{6}, O^{30}, O^{31}: O^{13}, O^{16}, O^{19}, O^{22}, O^{25}, O^{30}, O^{31}\right)$ )(nickel)barium $(2+)$ bis(trifluoromethanesulfonate) $(11)$ : red solid; yield $75 \%$; $\mathrm{mp}$ $>300{ }^{\circ} \mathrm{C}\left(\mathrm{CH}_{3} \mathrm{CN} / \mathrm{DIP}\right) ;{ }^{1} \mathrm{H}$ NMR (DMSO- $\left.d_{6}\right) \delta 8.04,7.97(\mathrm{~s}, 1 \mathrm{H}$ $\mathrm{N}=\mathrm{CH}), 7.3-7.1(\mathrm{~m}, 4 \mathrm{H}, \mathrm{Ar} \mathrm{H}), 6.8-6.6(\mathrm{~m}, 2 \mathrm{H}, \mathrm{Ar} \mathrm{H}), 4.4-4.2(\mathrm{~m}$, $\left.4 \mathrm{H}, \mathrm{ArOCH}_{2}\right), 4.0-3.8\left(\mathrm{~m}, 4 \mathrm{H}, \mathrm{OCH}_{2}\right), 3.70\left(\mathrm{~s}, 8 \mathrm{H}, \mathrm{OCH}_{2}\right), 3.44(\mathrm{~s}$, $\left.2 \mathrm{H}, \mathrm{NCH}_{2}\right), 1.53\left(\mathrm{~s}, 6 \mathrm{H}, \mathrm{CH}_{3}\right)$; IR $(\mathrm{KBr}) 1625(\mathrm{~N}=\mathrm{C}) \mathrm{cm}^{-1}$; mass spectrum $m / e 542.153\left(\mathrm{M}^{+}-\mathrm{Ba}\left(\mathrm{CF}_{3} \mathrm{SO}_{3}\right)_{2}\right.$, calcd 542.156). Anal. Caled for $\mathrm{C}_{28} \mathrm{H}_{32} \mathrm{BaF}_{6} \mathrm{~N}_{2} \mathrm{NiO}_{13} \mathrm{~S}_{2} \cdot 0.5 \mathrm{CH}_{3} \mathrm{CN}: \quad \mathrm{C}, 34.86 ; \mathrm{H}, 3.38 ; \mathrm{N}$, 3.50. Found: C, $34.70 ; \mathrm{H}, 3.97 ; \mathrm{N}, 3.22$.

$\left(\mu\right.$-(5,5-Dimethyl-13,16,19,22-tetraoxa-3,6-diazatricyclo[21.3.1.1 ${ }^{8,12}$ octacosa-1 (27), 2,6,8,10,12(28), 23,25-octaene-27,28-diolato(2-)$\left.\left.N^{3}, N^{6}, O^{27}, O^{28}: O^{13}, O^{16}, O^{19}, O^{22}, O^{27}, O^{28}\right)\right)($ copper $)$ barium $(2+)$ bis (trifluoromethanesulfonate) (12): green solid; yield $82 \% ; \mathrm{mp}>300^{\circ} \mathrm{C}$ $\left(\mathrm{CH}_{3} \mathrm{CN} / \mathrm{DIP}\right)$; IR $(\mathrm{KBr}) 1629(\mathrm{~N}=\mathrm{C}) \mathrm{cm}^{-1}$; mass spectrum $\mathrm{m} / \mathrm{e}$ $503.121\left(\mathrm{M}^{+}-\mathrm{Ba}\left(\mathrm{CF}_{3} \mathrm{SO}_{3}\right)\right.$, calcd 503.124). Anal. Calcd for $\mathrm{C}_{26} \mathrm{H}_{28} \mathrm{BaCuF}_{6} \mathrm{~N}_{2} \mathrm{O}_{12} \mathrm{~S}_{2} \cdot \mathrm{CH}_{3} \mathrm{CN}: \mathrm{C}, 34.30 ; \mathrm{H}, 3.19 ; \mathrm{N}, 4.29$. Found: $\mathrm{C}$, $34.16 ; \mathrm{H}, 3.24 ; \mathrm{N}, 3.98$

( $\mu$-(5,5-Dimethyl-13,16,19,22,25-pentaoxa-3,6-diazatricyclo[24.3.1.1 ${ }^{8,12}$ ] hentriacontane-1 $(30), 2,6,8,10,12(31), 26,28$-octaene-30,31diolato $\left.(2-)-N^{3}, N^{6}, O^{30}, O^{31}: O^{13}, O^{16}, O^{19}, O^{22}, O^{25}, O^{30}, O^{31}\right)$ )(copper)barium $(2+)$ bis(trifluoromethanesulfonate) $(13)$ : green solid; yield $71 \%$; $\mathrm{mp}$ $>300^{\circ} \mathrm{C}\left(\mathrm{CH}_{3} \mathrm{CN} / \mathrm{DIP}\right) ; \mathrm{IR}(\mathrm{KBr}) 1633(\mathrm{~N}=\mathrm{C}) \mathrm{cm}^{-1}$; mass spectrum $m / e$ 547.146 $\left(\mathrm{M}^{+}-\mathrm{Ba}\left(\mathrm{CF}_{3} \mathrm{SO}_{3}\right)_{2}\right.$, calcd for $\left.\mathrm{C}_{26} \mathrm{H}_{32} \mathrm{CuN}_{2} \mathrm{O}_{6} 547.151\right)$.

$3,3^{\prime}-\left(\left(2,2^{\prime}, 2^{\prime \prime}\right.\right.$-Trimethoxy-5,5', $5^{\prime \prime}$-trimethyl $\left[1,1^{\prime}: 3^{\prime}, 1^{\prime \prime}\right.$-terphenyl]3,3"-diyl)bis(methyleneoxy))bis(2-hydroxybenzaldehyde) $(\mathbf{2 0})$. To a suspension of $\mathrm{NaH}(1.38 \mathrm{~g}, 57.7 \mathrm{mmol})$ in $10 \mathrm{~mL}$ of dry DMSO under argon was added a solution of $18(3.65 \mathrm{~g}, 26.2 \mathrm{mmol})$ in $5 \mathrm{~mL}$ of dry DMSO, dropwise as $20-25^{\circ} \mathrm{C}$. After the addition was completed, the solution was stirred for $1 \mathrm{~h}$, after which a solution of $19(7.20 \mathrm{~g}, 13.1$ $\mathrm{mmol}$ ) in $10 \mathrm{~mL}$ of dry DMSO was added dropwise while the temperature was kept between 20 and $25^{\circ} \mathrm{C}$. The resulting solution was stirred for a weekend. The reaction mixture was then poured into $300 \mathrm{~mL}$ of $\mathrm{H}_{2} \mathrm{O}$ and acidified with $6 \mathrm{M} \mathrm{HCl}$ to $\mathrm{pH}=1$. The aqueous solution was extracted with $\mathrm{CHCl}_{3}(3 \times 60 \mathrm{~mL})$. The combined organic layers were washed with $\mathrm{H}_{2} \mathrm{O}(3 \times 50 \mathrm{~mL})$, dried with $\mathrm{MgSO}_{4}$, and concentrated. The crude product was purified with column chromatography (silica gel, $\mathrm{CH}_{2} \mathrm{Cl}_{2}$ ), after which a foam resulted that contained also a small amount of unreacted 18. The foam was treated with $\mathrm{MeOH}$ to give pure 20 as a white solid: yield $23 \%$; mp $184-186^{\circ} \mathrm{C}\left(1: 1 \mathrm{CH}_{3} \mathrm{CN} / \mathrm{DIP}\right) ;{ }^{1} \mathrm{H}$ NMR $\delta 10.97(\mathrm{~s}, 2 \mathrm{H}, \mathrm{OH}), 9.94(\mathrm{~s}, 2 \mathrm{H}, \mathrm{CHO}), 7.3-7.1\left(\mathrm{~m}, 10 \mathrm{H}, \mathrm{Ar} \mathrm{H}, \mathrm{Ar}^{\prime}\right.$ $\left.\mathrm{H}, \mathrm{Ar}^{\prime \prime} \mathrm{H}\right), 7.0-6.8(\mathrm{~m}, 2 \mathrm{H}, \mathrm{Ar} \mathrm{H}), 5.24\left(\mathrm{~s}, 4 \mathrm{H}, \mathrm{ArCH}_{2}\right), 3.51(\mathrm{~s}, 6$ $\mathrm{H}$, outer $\left.\mathrm{OCH}_{3}\right), 3.21\left(\mathrm{~s}, 3 \mathrm{H}\right.$, inner $\left.\mathrm{OCH}_{3}\right), 2.37\left(\mathrm{~s}, 3 \mathrm{H}\right.$, inner $\left.\mathrm{CH}_{3}\right)$, $2.37\left(\mathrm{~s}, 6 \mathrm{H}\right.$, outer $\left.\mathrm{CH}_{3}\right) ;{ }^{13} \mathrm{C}$ NMR $\delta 196.3(\mathrm{~d}, \mathrm{CHO}), 153.7,153.2$ $152.2,147.5$ (s, $\left.\mathrm{Ar} \mathrm{C}-2,3, \mathrm{Ar}^{\prime} \mathrm{C}-1, \mathrm{Ar}^{\prime \prime} \mathrm{C}-1\right), 133.2,132.6,132.0,131.8$, 129.1, 121.1 (s, Ar C-1, $\left.\mathrm{Ar}^{\prime} \mathrm{C}-2,4,6, \mathrm{Ar}^{\prime \prime} \mathrm{C}-2,4\right), 132.3,131.5,129.5$, 125.0, 120.6, 119.5 (d, Ar C-4-6, Ar' C-3,5, Ar" C-3), 67.0 (t, $\mathrm{ArCH}_{2}$ ), 61.3, $60.6\left(\mathrm{q}, \mathrm{OCH}_{3}\right), 20.8,20.7\left(\mathrm{q}, \mathrm{CH}_{3}\right) ; \mathrm{IR}(\mathrm{KBr}) 3420(\mathrm{OH}), 1682$ $(\mathrm{C}=\mathrm{O}) \mathrm{cm}^{-1}$; mass spectrum $\mathrm{m} / \mathrm{e} 662.244\left(\mathrm{M}^{+}\right.$, calcd 662.252$)$. Anal. Calcd for $\mathrm{C}_{40} \mathrm{H}_{38} \mathrm{O}_{9}: \mathrm{C}, 72.49 ; \mathrm{H}, 5.78$. Found: $\mathrm{C}, 72.06 ; \mathrm{H}, 5.65$.

(39,40,41-Trimethoxy-12,17,22-trimethyl-25H-3,7:10,14:15,19:20,24:27,31-pentametheno- $9 \mathrm{H}-8,26,1,33$-benzodioxadiazacyclopentatriacontine-38,42-diol- $O^{8}, O^{26}, O^{38}, O^{39}, O^{40}, O^{41}, O^{42}$ ) barium (2+) Bis(trifluoromethanesulfonate) (22). To a refluxing solution of $\mathrm{Ba}\left(\mathrm{CF}_{3} \mathrm{SO}_{3}\right)_{2}$ $(0.53 \mathrm{~g}, 1.21 \mathrm{mmol})$ in $150 \mathrm{~mL}$ of $\mathrm{MeOH}$ were added both a solution of $20(0.80 \mathrm{~g}, 1.21 \mathrm{mmol})$ in a mixture of $65 \mathrm{~mL}$ of $\mathrm{CH}_{2} \mathrm{Cl}_{2} / \mathrm{MeOH}(1: 1)$ and a solution of $21(0.13 \mathrm{~g}, 1.21 \mathrm{mmol})$ in $65 \mathrm{~mL}$ of $\mathrm{MeOH}$, dropwise over a period of $3 \mathrm{~h}$. After the addition was completed, the orange reaction mixture was cooled to room temperature and concentrated to dryness. To the residue was added $30 \mathrm{~mL}$ of $\mathrm{CH}_{3} \mathrm{CN}$, and the mixture was stirred for $0.5 \mathrm{~h}$. The insoluble parts were filtered off, and to the clear orange filtrate was added $50 \mathrm{~mL}$ of DIP, dropwise with constant stirring. The resulting precipitate was filtered off. Two extra portions of $30 \mathrm{~mL}$ of DIP were added, and the precipitates were again filtered off. The three portions contained only the desired macrocyclic compound: $:^{20}$ orange solid; yield $53 \% ; \mathrm{mp} 195-220^{\circ} \mathrm{C} \mathrm{dec}\left(\mathrm{CHCl}_{3} / \mathrm{Et}_{2} \mathrm{O}\right) ;{ }^{1} \mathrm{H} \mathrm{NMR}$ $\delta 8.47(\mathrm{~s}, 2 \mathrm{H}, \mathrm{N}=\mathrm{CH}), 7.4-7.1(\mathrm{~m}, 14 \mathrm{H}, \mathrm{Ar} \mathrm{H}), 7.0-6.8(\mathrm{~m}, 2 \mathrm{H}, \mathrm{Ar}$ $\mathrm{H}), 5.61$ and $4.77\left(\mathrm{AB}-\mathrm{q}, 4 \mathrm{H}, J=11.4 \mathrm{~Hz}, \mathrm{ArCH}_{2}\right), 3.65(\mathrm{~s}, 6 \mathrm{H}$, outer $\left.\mathrm{OCH}_{3}\right), 3.01$ (s, $3 \mathrm{H}$, inner $\mathrm{OCH}_{3}$ ), 2.47 (s, $3 \mathrm{H}$, inner $\left.\mathrm{CH}_{3}\right), 2.33$ (s, $6 \mathrm{H}$, outer $\left.\mathrm{CH}_{3}\right)$; IR $(\mathrm{KBr}) 1623(\mathrm{~N}=\mathrm{C}) \mathrm{cm}^{-1}$. Anal. Calcd for $\mathrm{C}_{48} \mathrm{H}_{42} \mathrm{BaF}_{6} \mathrm{~N}_{2} \mathrm{O}_{13} \mathrm{~S}_{2} \cdot 0.5 \mathrm{CHCl}_{3}: \mathrm{C}, 47.34 ; \mathrm{H}, 3.52 ; \mathrm{N}, 2.28$. Found: $\mathrm{C}$, 47.06; H, 3.43; N, 2.02 .

General Procedure for the Preparation of 23-25. To a solution of 22 $(0.20 \mathrm{~g}, 0.17 \mathrm{mmol})$ in a mixture of $4 \mathrm{~mL}$ of $\mathrm{CHCl}_{3}$ and $4 \mathrm{~mL}$ of $\mathrm{MeOH}$ was added a solution of 1 equiv of $\mathrm{Ni}(\mathrm{OAc}){ }_{2} \cdot 4 \mathrm{H}_{2} \mathrm{O}(0.042 \mathrm{~g}), \mathrm{Cu}(\mathrm{O}$ Ac) ${ }_{2} \cdot \mathrm{H}_{2} \mathrm{O}(0.034 \mathrm{~g})$, or $\mathrm{Zn}(\mathrm{OAc})_{2} \cdot 2 \mathrm{H}_{2} \mathrm{O}(0.037 \mathrm{~g})$ in $3-5 \mathrm{~mL}$ of $\mathrm{MeOH}$, at room temperature. The resulting colored solution was stirred for 10 min, after which it was concentrated to dryness.

( $\mu$ - (39,40,41-Trimethoxy-12,17,22-trimethyl-25H-3,7:10,14:15,19: 20,24:27,31-pentametheno- $9 H-8,26,1,33$-benzodioxadiazacyclopentatriacontine-38,42-diolato(2-)- $N^{1}, N^{33}, O^{38}, O^{42}: O^{8}, O^{26}, O^{38}, O^{39}, O^{40}, O^{41}$, $\left.O^{42}\right)$ )(nickel)barium(2+) Bis(trifluoromethanesulfonate) (23). The resulting dark red residue was treated with some $\mathrm{MeOH} / \mathrm{Et}_{2} \mathrm{O}$ (1:2), and the obtained precipitate was filtered off: red solid; yield $67 \% ; \mathrm{mp}>300$ ${ }^{\circ} \mathrm{C}\left(\mathrm{CH}_{3} \mathrm{CN} / \mathrm{DIP}\right) ;{ }^{1} \mathrm{H}$ NMR (DMSO- $\left.d_{6}\right) \delta 8.11(\mathrm{bs}, 2 \mathrm{H}, \mathrm{N}=\mathrm{CH}$ ), 8.0-7.3 (m, $14 \mathrm{H}, \mathrm{Ar} \mathrm{H}), 7.0-6.8(\mathrm{~m}, 2 \mathrm{H}, \mathrm{Ar} \mathrm{H}), 5.54$ and 4.98 (AB-q, $\left.4 \mathrm{H}, J=11.4 \mathrm{~Hz}, \mathrm{ArCH}_{2}\right), 3.47\left(\mathrm{~s}, 6 \mathrm{H}\right.$, outer $\left.\mathrm{OCH}_{3}\right), 2.79(\mathrm{~s}, 3 \mathrm{H}$, inner $\left.\mathrm{OCH}_{3}\right), 2.39\left(\mathrm{~s}, 6 \mathrm{H}\right.$, outer $\left.\mathrm{CH}_{3}\right)$, (inner $\mathrm{CH}_{3}$ not localized); IR $(\mathrm{KBr}) 1615(\mathrm{~N}=\mathrm{C}) \mathrm{cm}^{-1}$; mass spectrum $\mathrm{m} / e 1077\left(\mathrm{M}^{+}-\mathrm{CF}_{3} \mathrm{SO}_{3}^{-}\right.$, calcd 1077). Anal. Calcd for $\mathrm{C}_{48} \mathrm{H}_{40} \mathrm{BaF}_{6} \mathrm{~N}_{2} \mathrm{NiO}_{13} \mathrm{~S}_{2}: \mathrm{C}, 46.99 ; \mathrm{H}, 3.29$; $\mathrm{N}, 2.28$. Found: $\mathrm{C}, 47.09 ; \mathrm{H}, 3.41 ; \mathrm{N}, 2.02$.

( $\mu$-(39,40,41-Trimethoxy-12,17,22-trimethyl-25H-3,7:10,14:15,19:20,24:27,31-pentametheno-9H-8,26,1,33-benzodioxadiazacyclopentatriacontine-38,42-diolato(2-)- $N^{1}, N^{33}, O^{38}, O^{42}: O^{8}, O^{26}, O^{38}, O^{39}, O^{40}, O^{41}$, $\left.O^{42}\right)$ )(copper)barium(2+) Bis(trifluoromethanesulfonate) (24). The obtained dark green residue was treated with some $\mathrm{MeOH} / \mathrm{Et}_{2} \mathrm{O}(1: 2)$, and the resulting precipitate was filtered off: green solid; yield $76 \%$; $\mathrm{mp}$ $>300^{\circ} \mathrm{C}\left(\mathrm{CH}_{3} \mathrm{CN} / \mathrm{DIP}\right): \mathrm{IR}(\mathrm{KBr}) 1614(\mathrm{~N}=\mathrm{C}) \mathrm{cm}^{-1}$; mass spectrum $m / e 1082\left(\mathrm{M}^{+}-\mathrm{CF}_{3} \mathrm{SO}_{3}^{-}\right.$, calcd 1082). Anal. Calcd for $\mathrm{C}_{48} \mathrm{H}_{40} \mathrm{BaCuF}_{6} \mathrm{~N}_{2} \mathrm{O}_{13} \mathrm{~S}_{2}: \mathrm{C}, 46.80 ; \mathrm{H}, 3.27 ; \mathrm{N}, 2.27$. Found: $\mathrm{C}, 46.94$; $\mathrm{H}, 3.32 ; \mathrm{N}, 2.15$

$(\mu-(39,40,41$-Trimethoxy-12,17,22-trimethyl-25H-3,7:10,14:15,19:20,24:27,31-pentametheno-9H-8,26,1,33-benzodioxadiazacyclopentatriacontine-38,42-diolato (2-)- $N^{1}, N^{33}, O^{38}, O^{42}: O^{8}, O^{26}, O^{38}, O^{39}, O^{40}, O^{41}$, $\left.O^{42}\right)$ )(zinc)barium (2+) Bis(trifluoromethanesulfonate) (25). The yellow residue was treated with some $\mathrm{MeOH} / \mathrm{Et}_{2} \mathrm{O}$ (1:3), and the resulting yellow precipitate was filtered off: yellow solid; yield $81 \% ; \mathrm{mp}>300^{\circ} \mathrm{C}$ $\left(\mathrm{CH}_{3} \mathrm{CN} / \mathrm{DIP}\right) ;{ }^{1} \mathrm{H}$ NMR (DMSO- $\left.d_{5}\right) \delta 9.18(\mathrm{~s}, 2 \mathrm{H}, \mathrm{N}=\mathrm{CH}), 8.1-8.0$ $(\mathrm{m}, 2 \mathrm{H}, \mathrm{Ar} \mathrm{H}), 7.6-7.2(\mathrm{~m}, 12 \mathrm{H}, \mathrm{Ar} \mathrm{H}), 6.9-6.7(\mathrm{~m}, 2 \mathrm{H}, \mathrm{Ar} \mathrm{H}), 5.43$ and $5.09\left(\mathrm{AB}-\mathrm{q}, 4 \mathrm{H}, J=12.2 \mathrm{~Hz}, \mathrm{ArCH}_{2}\right), 3.47\left(\mathrm{~s}, 6 \mathrm{H}\right.$, outer $\left.\mathrm{OCH}_{3}\right)$ $289\left(\mathrm{~s}, 3 \mathrm{H}\right.$, inner $\left.\mathrm{OCH}_{3}\right), 240\left(\mathrm{~s}, 6 \mathrm{H}\right.$, outer $\mathrm{CH}_{3}$ ) (inner $\mathrm{CH}_{3}$ not localized); IR $(\mathrm{KBr}) 1615(\mathrm{~N}=\mathrm{C}) \mathrm{cm}^{-1}$; mass spectrum m/e $1083\left(\mathrm{M}^{+}\right.$ - $\mathrm{CF}_{3} \mathrm{SO}_{3}^{-}$, calcd 1083). Anal. Calcd for $\mathrm{C}_{48} \mathrm{H}_{40} \mathrm{BaF}_{6} \mathrm{~N}_{2} \mathrm{O}_{13} \mathrm{~S}_{2} \mathrm{Zn}$ : C, $46.73 ; \mathrm{H}, 3.27 ; \mathrm{N}, 2.27$. Found: C, 46.85; H, 3.37; N, 2.20 . 
General Procedure for the Synthesis of the Complexes 26 and 27 . A suspension of the dinuclear complexes $23(0.14 \mathrm{~g})$ and $24(0.16 \mathrm{~g})$, respectively, in $\mathrm{CHCl}_{3}(50 \mathrm{~mL})$ was vigorously stirred for $0.5 \mathrm{~h}$ with a solution of guanidinium sulfate $\left(\mathrm{Gu}_{2} \mathrm{SO}_{4}\right.$, excess) in $30 \mathrm{~mL}$ of $\mathrm{H}_{2} \mathrm{O}$. The resulting colored organic solution was separated off and filtered. After removal of the solvent the mononuclear barium-free complex was obtained in quantitative yield.

(39,40,41-Trimethoxy-12,17,22-trimethyl-25H-3,7:10,14:15,19:20, 24:27,31-pentametheno- $9 H-8,26,1,33$-benzodioxadiazacyclopentatriacontine-38,42-diolato $\left.(2-)-N^{1}, N^{33}, O^{38}, O^{42}\right)$ nickel (26): red solid; mp 250 ${ }^{\circ} \mathrm{C} \mathrm{dec}\left(\mathrm{CHCl}_{3} /\right.$ petroleum ether 40-60); ${ }^{1} \mathrm{H}$ NMR $\delta 7.71$ (bs, $2 \mathrm{H}$ $\mathrm{N}=\mathrm{CH}), 7.3-6.8(\mathrm{~m}, 14 \mathrm{H}, \mathrm{Ar} \mathrm{H}), 6.8-6.5(\mathrm{~m}, 2 \mathrm{H}, \mathrm{Ar} \mathrm{H}), 5.41$ and 4.20 (AB-q, $\left.4 \mathrm{H}, J=11.4 \mathrm{~Hz}, \mathrm{ArCH}_{2}\right), 3.46\left(\mathrm{~s}, 6 \mathrm{H}\right.$, outer $\left.\mathrm{OCH}_{3}\right), 2.57$ (s, $3 \mathrm{H}$, inner $\left.\mathrm{OCH}_{3}\right), 2.43\left(\mathrm{~s}, 3 \mathrm{H}\right.$, inner $\left.\mathrm{CH}_{3}\right), 2.31\left(\mathrm{~s}, 6 \mathrm{H}\right.$, outer $\left.\mathrm{CH}_{3}\right)$ IR $(\mathrm{KBr}) 1610(\mathrm{~N}=\mathrm{C}) \mathrm{cm}^{-1}$; mass spectrum $m / e 791\left(\mathrm{M}^{+}+\mathrm{H}\right.$, calcd 791). Anal. Calcd for $\mathrm{C}_{46} \mathrm{H}_{40} \mathrm{~N}_{2} \mathrm{NiO}_{7} \cdot \mathrm{CHCl}_{3}: \mathrm{C}, 61.97 ; \mathrm{H}, 4.54 ; \mathrm{N}$, 3.08. Found: C, $62.09 ; \mathrm{H}, 5.04 ; \mathrm{N}, 2.91$.

(39,40,41-Trimethoxy-12,17,22-trimethyl-25H-3,7:10,14:15,19:20, 24:27,31-pentametheno- $9 H-8,26,1,33$-benzodioxadiazacyclopentatriacontine-38,42-diolato (2-)- $\left.N^{1}, N^{33}, O^{38}, O^{42}\right)$ copper (27): green solid; $\mathrm{mp}$ $240{ }^{\circ} \mathrm{C}$ dec $\left(\mathrm{CHCl}_{3} /\right.$ petroleum ether 40-60); IR $(\mathrm{KBr}) 1610(\mathrm{~N}=\mathrm{C})$ $\mathrm{cm}^{-1}$; mass spectrum $\mathrm{m} / e \mathrm{e} 96\left(\mathrm{M}^{+}+\mathrm{H}\right.$, calcd 796). Anal. Calcd for $\mathrm{C}_{46} \mathrm{H}_{40} \mathrm{CuN}_{2} \mathrm{O}_{7} \cdot 0.8 \mathrm{CHCl}_{3}: \mathrm{C}, 63.03 ; \mathrm{H}, 4.61 ; \mathrm{N}, 3.14$. Found: $\mathrm{C}, 62.87 ;$ $\mathrm{H}, 4.93 ; \mathrm{N}, 3.04$.

( $\mu$ - $(37,38,39-T$ rimethoxy-5,5,37,38,39-pentamethyl-13,31-dioxa-3,6diazahexacyclo $\left[30.3 .1 .1^{8,12} .1^{15,19} .1^{20,24} .1^{25,29}\right]$ tetraconta-1$(36), 2,6,8,10,12(40), 15,17,19(39), 20,22,24(38), 25,27,29(37), 32,34$-heptadecaene-36,40-diolato(2-)- $N^{3}, N^{6}, O^{36}, O^{40}: O^{13}, O^{31}, O^{36}, O^{37}, O^{38}, O^{39}$, $\left.\mathrm{O}^{40}\right)$ )(nickel)barium(2+) Bis(trifluoromethanesulfonate) (28). To a refluxing solution of $\mathrm{Ba}\left(\mathrm{CF}_{3} \mathrm{SO}_{3}\right)_{2}(0.45 \mathrm{~g}, 1.04 \mathrm{mmol})$ in $150 \mathrm{~mL}$ of $\mathrm{MeOH}$ were added both a solution of $20(0.69 \mathrm{~g}, 1.04 \mathrm{mmol})$ in $50 \mathrm{~mL}$ of $\mathrm{CH}_{2} \mathrm{Cl}_{2} / \mathrm{MeOH}(1: 1)$ and a solution of $9(0.092 \mathrm{~g}, 1.04 \mathrm{mmol})$ in 50 $\mathrm{mL}$ of $\mathrm{MeOH}$, dropwise over a period of $2.5 \mathrm{~h}$. After the addition was completed, the yellow solution was refluxed for $10 \mathrm{~min}$, after which a solution of $\mathrm{Ni}(\mathrm{OAc})_{2}, 4 \mathrm{H}_{2} \mathrm{O}(0.26 \mathrm{~g}, 1.04 \mathrm{mmol})$ in $20 \mathrm{~mL}$ of $\mathrm{MeOH}$ was added in one portion. The resulting red solution was cooled to room temperature and concentrated to $50 \mathrm{~mL}$. A precipitate was formed, which was filtered off. To the filtrate was added $50 \mathrm{~mL}$ of DIP, and the precipitate formed was filtered off. This was repeated with a portion of $100 \mathrm{~mL}$ of DIP. These three precipitates contained noncyclic product. The ultimate filtrate was concentrated to dryness and dissolved in $5 \mathrm{~mL}$ of $\mathrm{CH}_{3} \mathrm{CN}$. After the addition of $30 \mathrm{~mL}$ of DIP the dinuclear complex 28 precipitated and was filtered off: $2^{21}$ red solid; yield $53 \% ; \mathrm{mp} 280^{\circ} \mathrm{C}$ $\operatorname{dec}\left(\mathrm{CH}_{3} \mathrm{CN} / \mathrm{EtOH} / \mathrm{DIP}\right) ;{ }^{1} \mathrm{H}$ NMR $\left(\mathrm{CD}_{3} \mathrm{CN}\right) \delta 7.62$ (s, $2 \mathrm{H}, \mathrm{N}=$ $\mathrm{CH}), 7.4-7.3(\mathrm{~m}, 8 \mathrm{H}, \mathrm{Ar} \mathrm{H}), 7.1-7.0(\mathrm{~m}, 2 \mathrm{H}, \mathrm{Ar} \mathrm{H}), 6.9-6.8(\mathrm{~m}, 2$ $\mathrm{H}, \mathrm{Ar} \mathrm{H}$ ), 5.54 and $4.96,4.92$ (AB-q, $4 \mathrm{H}, J=12.1 \mathrm{~Hz}, \mathrm{ArCH}_{2}$ ), 3.52 $\left(\mathrm{s}, 6 \mathrm{H}\right.$, outer $\left.\mathrm{OCH}_{3}\right), 3.3-3.2\left(\mathrm{~m}, 2 \mathrm{H}, \mathrm{NCH}_{2}\right), 3.06(\mathrm{~s}, 3 \mathrm{H}$, inner $\left.\mathrm{OCH}_{3}\right), 2.48\left(\mathrm{~s}, 3 \mathrm{H}\right.$, inner $\left.\mathrm{CH}_{3}\right), 2.37\left(\mathrm{~s}, 6 \mathrm{H}\right.$, outer $\left.\mathrm{CH}_{3}\right), 1.47,1.43$ (s, $\left.3 \mathrm{H}, \mathrm{NC}\left(\mathrm{CH}_{3}\right)_{2}\right) ; \mathrm{IR}(\mathrm{KBr}) 1625(\mathrm{C}=\mathrm{N}) \mathrm{cm}^{-1}$. Anal. Calcd for $\mathrm{C}_{46} \mathrm{H}_{44} \mathrm{BaF}_{6} \mathrm{~N}_{2} \mathrm{NiO}_{13} \mathrm{~S}_{2} \cdot \mathrm{EtOH}: \mathrm{C}, 46.01 ; \mathrm{H}, 4.02 ; \mathrm{N}, 2.24$. Found: $\mathrm{C}$, $46.22 ; \mathrm{H}, 4.16 ; \mathrm{N}, 2.11$

(37,38,39-Trimethoxy-5,5,37,38,39-pentamethyl-13,31-dioxa-3,6-diazahexacyclo[30.3.1.1 $\left.1^{8,12} \cdot 1^{15,19} \cdot 1^{20,24} \cdot 1^{25,29}\right]$ tetraconta-1(36), 2,6,8,10,12$(40), 15,17,19(39), 20,22,24(38), 25,27,29(37), 32,34$-heptadecaene-36,40. diolato (2-)- $\left.N^{3}, N^{5}, O^{36}, O^{40}\right)$ (nickel) (29). A suspension of $28(0.30 \mathrm{~g})$ in $\mathrm{CHCl}_{3}(50 \mathrm{~mL})$ was stirred with a solution of $\mathrm{Gu}_{2} \mathrm{SO}_{4}$ (excess) in 30 $\mathrm{mL}$ of water until the organic layer was clear. It was separated off and filtered, followed by evaporation to dryness. The barium-free complex was obtained in quantitative yield: red solid; $\mathrm{mp}>300^{\circ} \mathrm{C}\left(\mathrm{CH}_{3} \mathrm{CN} /\right.$ DIP); ${ }^{1} \mathrm{H}$ NMR $\delta 7.37(\mathrm{~s}, 2 \mathrm{H}, \mathrm{N}=\mathrm{CH}), 7.3-6.9(\mathrm{~m}, 8 \mathrm{H}, \mathrm{Ar} \mathrm{H})$, 6.8-6.4 (m, $4 \mathrm{H}, \mathrm{Ar} \mathrm{H}), 5.42$ and $4.65,4.62(\mathrm{AB}-\mathrm{q}, 4 \mathrm{H}, J=11.4 \mathrm{~Hz}$, $\left.\mathrm{ArCH}_{2}\right), 3.50\left(\mathrm{~s}, 6 \mathrm{H}\right.$, outer $\left.\mathrm{OCH}_{3}\right), 3.12\left(\mathrm{~s}, 2 \mathrm{H}, \mathrm{NCH}_{2}\right), 2.67(\mathrm{~s}, 3 \mathrm{H}$, inner $\left.\mathrm{OCH}_{3}\right), 2.43\left(\mathrm{~s}, 3 \mathrm{H}\right.$, inner $\left.\mathrm{CH}_{3}\right), 2.32\left(\mathrm{~s}, 6 \mathrm{H}\right.$, outer $\left.\mathrm{CH}_{3}\right), 1.35$, $1.29\left(\mathrm{~s}, 3 \mathrm{H}, \mathrm{NC}\left(\mathrm{CH}_{3}\right)_{2}\right) ; \mathrm{IR}(\mathrm{KBr}) 1622(\mathrm{~N}=\mathrm{C}) \mathrm{cm}^{-1}$; mass spectrum $m / e$ $770.245\left(\mathrm{M}^{+}\right.$, calcd 770.250$)$

$\mathrm{X}$-ray Crystallography. X-ray diffraction measurements were performed on a Philips PW 1100 or an Enraf-Nonius CAD4 diffractometer, using graphite-monochromated Mo $\mathrm{K} \alpha(\lambda=0.71073 \AA)$ radiation. Crystal data and data collection parameters are collected in Table II. Lattice parameters were determined by least squares from 25 centered reflections. Intensities were measured in the $\omega / 2 \theta$ scan mode and are corrected for decay of three control reflections (for all four structures less than 5\%), measured every hour, and for Lorentz and polarization factors.

The metal ions were located by Patterson methods and the rest of the heavy atoms by successive difference Fourier syntheses. Reflections with $F_{0}{ }^{2}>3 \sigma\left(F_{0}{ }^{2}\right)$ were considered observed and were included in the refinement (on $F$ ) by full-matrix least squares. Weights were calculated as $w=4 F_{0}^{2} / \sigma^{2}\left(F_{0}^{2}\right)$, with $\sigma^{2}\left(F_{0}^{2}\right)=\sigma^{2}(I)+\left(p F_{0}^{2}\right)^{2}, \sigma(I)$ being based on counting statistics and $p$ being an instability factor obtained from plots of $F_{0}$ vs weighted error. In all structures the metal ions were refined with anisotropic thermal parameters, and depending on the ratio of data:parameters and the presence of disorder, other atoms were also refined anisotropically. In the case of $14 \cdot \mathrm{Na}^{+} \mathrm{Pic}^{-}$(dark red crystals) one carbon atom of the polyethylene glycol chain was found to be disordered over two positions, which were refined with an occupancy factor of 0.5 . The noncoordinating anion in 25.3DMF (pale yellow crystals) was located at two positions: near a center of symmetry and near a 2 -fold axis of symmetry. They were both highly disordered. In order to mimic this, they were refined as " $\mathrm{FO}_{3}$ " ( $\mathrm{F}$ was used to mimic the assumed 50/50 mixture of $\mathrm{C}$ and $\mathrm{S}$ ). For 3.MeOH (red crystals) and 25.3DMF an empirical absorption correction, using DIFABS, ${ }^{43}$ was performed; relative correction factors were $0.9155-1.0325$ for $3 \cdot \mathrm{MeOH}$ and $0.8502-1.1275$ for 25.3DMF. In all structures the hydrogen atoms were placed on their calculated positions and for the compounds $3 \cdot \mathrm{MeOH}, 14 \cdot \mathrm{Na}^{+} \mathrm{Pic}^{-}$, and 25.3DMF treated as riding on their parent carbon atoms. In the case of $10 \cdot \mathrm{H}_{2} \mathrm{O}$ (ruby red crystals) the hydrogens were refined except for three, which were treated as riding on their parent carbons. Parameters refined were the overall scale factor, the isotropic extinction parameter $g\left[F_{\mathrm{o}}=F_{\mathrm{c}} /\left(1+g I_{\mathrm{c}}\right)\right]$ for $\mathbf{3} \cdot \mathrm{MeOH}$ and $\mathbf{1 0} \cdot \mathrm{H}_{2} \mathrm{O}$, positional and isotropic or anisotropic thermal parameters for non-hydrogen atoms, and positional and isotropic thermal parameters for hydrogen atoms (if included). Refinements converged with shift:error ratios less than unity, except for 25.3DMF, where convergence in the parameters of the disordered anion was poor. Final difference Fourier maps showed no significant features. All calculation were done by using SDP. 44,45

Electrochemistry. The polarographic measurements were carried out with a Metrohm E505 polarograph. This polarograph was operated in a three-electrode mode with a dropping mercury electrode (DME) as cathode, a platinum wire as auxiliary electrode, and an $\mathrm{Ag} / \mathrm{AgCl}$ electrode (Metrohm EA 441/5) as reference. The reference electrode was filled with $1 \mathrm{M} \mathrm{Et}_{4} \mathrm{~N}^{+} \mathrm{Cl}^{-}$(Merck, synthetic quality, recrystallized from ethyl acetate $/ \mathrm{CHCl}_{3}$ ) in $\mathrm{MeOH}$ (Merck, pA quality). The measurements were done at $20^{\circ} \mathrm{C}$ in a $0.1 \mathrm{M}$ solution of TEAP $\left(\mathrm{Et}_{4} \mathrm{~N}^{+} \mathrm{ClO}_{4}^{-}\right.$, Fluka, purum, recrystallized from EtOH) in DMSO (Merck, pA quality, maximum $0.03 \% \mathrm{H}_{2} \mathrm{O}$ ). The reference electrode was brought into contact with the sample via a double salt bridge of the configuration

\section{Ag; $\mathrm{AgCl}, \mathrm{Et}_{4} \mathrm{~N}^{+} \mathrm{Cl}^{-}-\mathrm{MeOH}$ :TEAP-DMSO:sample}

The characteristics of the DME electrode were $m=1.065 \mathrm{mg} / \mathrm{s}$, natural drop time $5.30 \mathrm{~s}$, and height of the mercury column $64 \mathrm{~cm}$. A mechanical drop time of $1.000 \mathrm{~s}$ was maintained during all experiments. The sample concentrations were $0.96-3.64 \mathrm{mM}$. Oxygen was expelled by bubbling with nitrogen (Hoekloos, very pure) for at least $10 \mathrm{~min}$. Polarograms were recorded and evaluated by a computerized method described by Zollinger et al. ${ }^{33}$ Compound 10 was used as reference compound and measured several times during the day to detect fluctuations $\left(\Delta E_{1 / 2}<\right.$ $3 \mathrm{mV})$.

Cyclic voltammetry was carried out with an Autolab computerized system for electrochemistry (Eco Chemie, Utrecht, The Netherlands). The measurements were performed at a stationary hanging mercury drop electrode (Metrohm, 663 VA stand). The same reference and auxiliary electrode were used as in the polarographic experiments. The solvent and the supporting electrolyte were also the same as used in the polarography.

Acknowledgment. We thank Akzo International Research BV for financial support and Professor N. Nibbering and R. R. Fokkens (University of Amsterdam, The Netherlands) for recording the FAB mass spectra.

Registry No. 1, 113063-80-2; 2, 115161-55-2; 3, 115161-57-4; 3 . $\mathrm{MeOH}, 119207-45-3 ; 4,115161-49-4 ; 5,115161-51-8 ; 6,108036-08-4$; $7,115142-66-0 ; 8,115142-67-1 ; 9,811-93-8 ; 10,119207-32-8 ; 10 \cdot \mathrm{H}_{2} \mathrm{O}$ $119207-53-3 ; 11,119207-47-5 ; 12,119243-53-7 ; 13,119207-49-7 ; 14$, $115161-69-8 ; 14 \cdot \mathrm{Na}^{+} \mathrm{Pic}^{-}, 119207-51-1 ; 14 \cdot \mathrm{K}^{+}, 119207-55-5 ; 14 \cdot \mathrm{Na}^{+}$ $119207-56-6 ; 14 \cdot \mathrm{Li}^{+}, 119207-57-7 ; 14 \cdot \mathrm{Rb}^{+}, 119207-70-4 ; 14 \cdot \mathrm{Cs}^{+}$, $119207-71-5 ; 15,115161-73-4 ; 15 \cdot \mathrm{K}^{+}, 119207-58-8 ; 15 \cdot \mathrm{Na}^{+}, 119207-$ $59-9 ; 15 \cdot \mathrm{Li}^{+}, 119207-60-2 ; 16,115161-71-2 ; 16 \cdot \mathrm{K}^{+} \mathrm{Pic}^{-}, 119207-69-1$; 16.K $\mathrm{K}^{+}, 119207-61-3 ; 16 \cdot \mathrm{Na}^{+}, 119207-62-4 ; 16 \cdot \mathrm{Li}^{+}, 119207-63-5 ; 16 \cdot \mathrm{Rb}^{+}$, $119207-72-6 ; 16 \cdot \mathrm{Cs}^{+}, 119207-73-7 ; 17,115161-74-5 ; 17 \cdot \mathrm{K}^{+}, 119207-$ $64-6 ; 17 \cdot \mathrm{Na}^{+}, 119207-74-8 ; 17 \cdot \mathrm{Li}^{+}, 119207-65-7 ; 18,24677-78-9 ; 19$, 71128-93-3; 20, $119182-86-4 ; \quad 21,95-54-5 \cdot 22,119207-34-0 ; 23$ 119207-36-2; 24, 119207-38-4; 25, 119243-51-5; 25.3DMF, 119243-55-9; 26, $119207-39-5 ; 26 \cdot \mathrm{K}^{+}, 119207-66-8 ; 27,119207-40-8 ; 27 \cdot \mathrm{K}^{+}$,

(43) Walker, N.; Stuart, D. Acta Crystallogr., Sect. A 1983, 39, 158

(44) "Structure Determination Package"; B. A. Frenz and Associates, College Station, TX, and Enraf-Nonius, Delft, The Netherlands, 1983.

(45) Scattering factors in SDP are taken from: (a) Cromer, D. T.; Waber, J. T. International Tables for X-ray Crystallography; Kynoch Press: Birmingham, England, 1974; Vol. IV, Table 2.2B. (b) Cromer, D. T.; Mann, J. B. Acta Crystallogr., Sect. A 1968, 24, 321 
$119207-67-9 ; 28,119207-42-0 ; 29,119207-54-4 ; 29 \cdot \mathrm{K}^{+}, 119207-68-0$; $\mathrm{Gu}_{2} \mathrm{SO}_{4}, 594-14-9$.

Supplementary Material Available: Tables S1.1-1.4, listing positional and isotropic thermal parameters, Tables S2.1-2.4, listing bond distances, Tables S3.1-3.4, listing bond angles, Table S4.1-4.4, listing bond distances of the coordinated transition-metal cations, Tables S5.1-5.4, listing bond angles of the coordinated transition-metal cations, Tables S6.1-6.4, listing anisotropic thermal parameters, and Tables S7.1-7.4, listing least-squares planes and deviations therefrom, and figures showing the structures, respectively, for the complexes $3 \cdot \mathrm{MeOH}, 10 \cdot \mathrm{H}_{2} \mathrm{O}, 14 \cdot \mathrm{Na}^{+} \mathrm{Pic}^{-}$, and 25.3DMF ( 37 pages); Tables S8.1-8.4, listing calculated and observed structure factors (120 pages). Ordering information is given on any current masthead page.

Contribution from the Department of Chemistry, Harvard University, Cambridge, Massachusetts 02138

\title{
Chemical and Electrochemical Reactivity of Nickel(II,I) Thiolate Complexes: Examples of Ligand-Based Oxidation and Metal-Centered Oxidative Addition
}

\author{
H.-J. Krüger and R. H. Holm*
}

Received September 29, 1988

\begin{abstract}
The first extensive study of the reactivity of nickel in a classical coordination environment containing mainly thiolate ligands is described and was undertaken to examine the factors affecting the stability of $\mathrm{Ni}(\mathrm{III})$ in hydrogenases. Reaction of $\mathrm{Ni}(\mathrm{acac})_{2}$ with pyridine-2,6-dimethanethiol $\left(\mathrm{H}_{2} \mathrm{pdmt}\right)$ in toluene afforded dimeric $[\mathrm{Ni}(\mathrm{pdmt})]_{2}(1)$. This compound can be electrochemically reduced at $E_{1 / 2}=-1.21 \mathrm{~V}$ vs SCE in DMF solution to [Ni(pdmt) $]_{2}^{-}\left(\mathbf{1}^{-}\right)$, a mixed-valence $\mathrm{Ni}(\mathrm{II}, \mathrm{I})$ complex, and can be cleaved with thiolate to afford $[\mathrm{Ni}(\mathrm{pdmt})(\mathrm{SR})]^{-}(\mathrm{R}=\mathrm{Ph}(2)$, Et $(3))$. $\left(n-\mathrm{Bu}_{4} \mathrm{~N}\right)[2]$ crystallizes in the monoclinic space group $P 2_{1} / a$ with $a=15.256(7) \AA, b=9.610(4) \AA, c=21.340(9) \AA, \beta=95.15(4)^{\circ}$, and $Z=4$. ( $\left.\mathrm{Me}_{4} \mathrm{~N}\right)[3]$ crystallizes in the monoclinic space group $P 2_{1} / n$ with $a=7.628$ (4) $\AA, b=20.014$ (8) $\AA, c=12.800$ (4) $\AA, \beta=98.53$ (4) ${ }^{\circ}$, and $Z=4$. Both complexes are planar with unexceptional metric features. Complexes 2 and 3 undergo the irreversible ligand-based oxidation $[\mathrm{Ni}(\mathrm{pdmt})(\mathrm{SR})]^{-} \rightarrow 1 / 2[1]$ $+1 / 2 \mathrm{RSSR}+\mathrm{e}^{-}$at $E_{\mathrm{pa}}=+0.01(2)$ and $-0.08(3) \mathrm{V}$. The reverse of this reaction was also demonstrated. The reduced dimer exhibits the oxidative-addition reaction $\mathbf{1}^{-}+\operatorname{RSSR} \rightarrow[2 / 3]+1 / 2[1]+1 / 2 R S S R$. Plausible pathways for these reactions are presented. These results and related observations show that ligand-based oxidation of classical nickel(II) thiolate complexes with irreversible formation of disulfide is a pervasive process that extends to unidentate and chelating thiolate ligands. Nickel sites in certain hydrogenases contain ca. three anionic sulfur ligands and are characterized by extremely low $\mathrm{Ni}$ (III)/Ni(II) potentials. Factors contributing to the stability of the Ni(III) enzyme sites are considered, and the general type of ligand that should be effective in stabilizing $\mathrm{Ni(III)}$ at low potentials is described.
\end{abstract}

\section{Introduction}

The purposeful exploration of structures and reactions of nickel thiolate complexes ${ }^{1-5}$ has most recently been motivated by the discovery of nickel in a large number of hydrogenases. ${ }^{6}$ The EPR signals with $g$ values near $2.3,2.2$, and 2.0 usually observed in most "as prepared" hydrogenases have been ascribed to Ni(III) in a tetragonal environment. Recent MCD results, which indicate a dissimilarity in coordination geometry of paramagnetic, distorted tetrahedral $\mathrm{Ni}(\mathrm{II})$-substituted rubredoxins vs diamagnetic $\mathrm{Ni}(\mathrm{II})$ in hydrogenases, ${ }^{7}$ add further support to tetragonal coordination in the latter. However, the more provocative property of these centers is their low Ni(III/II) redox potentials of ca. -150 to -400 $\mathrm{mV}$ vs NHE $(-390$ to $-640 \mathrm{mV}$ vs SCE), values remarkably depressed compared to the normal values of ca. $+500 \mathrm{mV}$ or higher vs SCE for synthetic complexes. ${ }^{8}$ There are a few such complexes

(1) Snyder, B. S. Rao, Ch. P. Holm, R. H. Aust. J. Chem. 1986, 39,963

(2) Krüger, H.-J.; Holm, R. H. Inorg. Chem. 1987, 26, 3645; unpublished results.

(3) Yamamura, T.; Miyamae, H.; Katayama, Y.; Sasaki, Y. Chem. Lett. 1985, 269.

(4) Yamamura, T. Chem. Lett. 1986, 801

(5) (a) Rosenfield, S. G.; Armstrong, W. H.; Mascharak, P. K. Inorg. Chem. 1986, 25, 3014. (b) Rosenfield, S. G.; Wong, M. L. Y.; Stephan, D. W.; Mascharak, P. K. Inorg. Chem. 1987, 26, 4119.

(6) (a) Hausinger, R. P. Microbiol. Rev. 1987, 5i, 22. (b) Cammack, R. Adv. Inorg. Chem. 1988, 32, 297.

(7) Kowal, A. T.; Zambrano, I. C.; Moura, I.; Moura, J. J. G.; LeGall, J.; Johnson, M. K. Inorg. Chem. 1988, $27,1162$.

(8) (a) Nag, K.; Chakravorty, A. Coord. Chem. Rev. 1980, 33, 87. (b) Haines, R. J.; McAuley, A. Coord. Chem. Rev. 1981, 39, 77 . (c) Lappin, A. G.; McAuley, A. Adv. Inorg. Chem. 1988, 32, 241. with substantially lower potentials than the latter value. $2,9,10$ Several of these, prepared in this laboratory, are classical amide thiolate complexes with potentials in the $-330-$ to $-35-m V$ interval., ${ }^{2,1}$ These results are of apparent relevance to the enzymes inasmuch as the Ni EXAFS of the latter have been interpreted in terms of (at least) three anionic sulfur ligands. ${ }^{12}$ One means of lowering $\mathrm{Ni}(\mathrm{III} / \mathrm{II})$ potentials, therefore, is to incorporate around the metal negative, polarizable ligands.

A promising initial model for the $\mathrm{Ni}$ site in hydrogenases would be a mononuclear tetragonal complex containing three or more thiolate ligands and exhibiting a low, reversible Ni(III/II) potential. Because of the highly anisotropic EPR spectra of the enzymes, a dithiolene-type nickel cofactor appears to be an unlikely candidate for the model. ${ }^{13}$ Certain difficulties attend the preparation of such model complexes. One is the decided tendency of nickel(II) thiolates to form polynuclear complexes, ${ }^{1,3,4,56,14,15}$ especially in protic media. Two types of mononuclear thiolates are known. The first and more common, $\left[\mathrm{Ni}(\mathrm{SR})_{4}\right]^{2-}$, is derived

(9) Busch, D. H. Acc. Chem. Res. 1978, 11, 392.

(10) Chakravorty, A. Isr. J. Chem. 1985, 25, 99.

(11) Hereafter, all potentials are referenced to the SCE.

(12) (a) Lindahl, P. A.; Kojima, N.; Hausinger, R. P.; Fox, J. A.; Teo, B. K.; Walsh, C. T.; Orme-Johnson, W. H. J. Am. Chem. Soc. 1984, lo6, 3062. (b) Scott, R. A.; Wallin, S. A.; Czechowski, M.; DerVartanian, D. V.; LeGall, J.; Peck, H. D., Jr.; Moura, I. J. Am. Chem. Soc. 1984, 106, 6864. (c) Scott, R. A.; Czechowski, M.; DerVartanian, D. V.; LeGall, J.; Peck, H. D., Jr.; Moura, I. Rev. Port. Quim. 1985, 27, 67.

(13) The EPR spectra of $\left[\mathrm{Ni}\left(\mathrm{S}_{2} \mathrm{C}_{2} \mathrm{R}_{2}\right)_{2}\right]^{1-}$ complexes are much less anisotropic; cf., e.g.: Maki, A. H.; Edelstein, N.; Davison, A.; Holm, R. H. $J$. Am. Chem. Soc. 1964, 86, 4580

(14) Dance, I. G. Polyhedron 1986, 5, 1037.

(15) Blower, P. J.; Dilworth, J. R. Coord. Chem. Rev. 1987, 76, 121. 